Aus dem Bereich Psychoanalyse, Psychotherapie und Psychosomatische Medizin Universitätsklinikum des Saarlandes, Homburg/Saar

Prof. Dr. med. habil. V. Köllner

\title{
Verbesserung des Transfers von Progressiver Relaxation in den Alltag der Patienten nach stationärer psychosomatischer Rehabilitation
}

\author{
Dissertation zur Erlangung des Grades eines Doktors der Medizin \\ (der Theoretischen Medizin, der Zahnheilkunde oder der Naturwissenschaften) \\ der Medizinischen Fakultät \\ der UNIVERSITÄT DES SAARLANDES
}

2012

vorgelegt von: Stefanie Franziska Klosterhalfen geb. am: 25.10.1985 in Neunkirchen/Saar 


\section{Inhaltsverzeichnis}

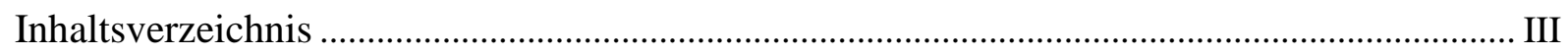

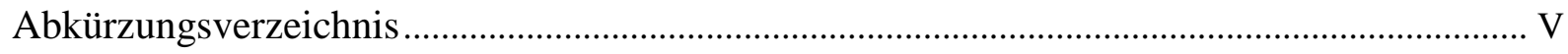

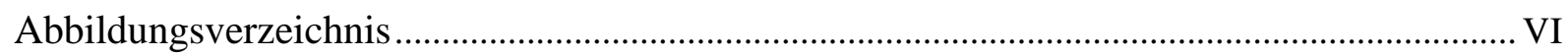

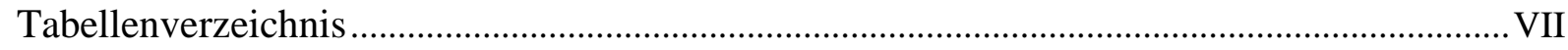

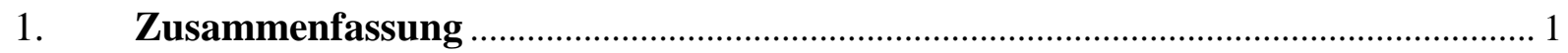

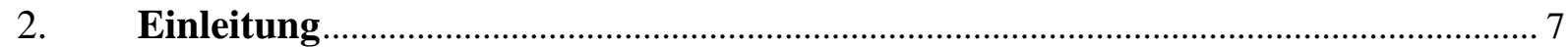

2.1 Wirkungsweise und Indikationen von Entspannungsverfahren..................................... 8

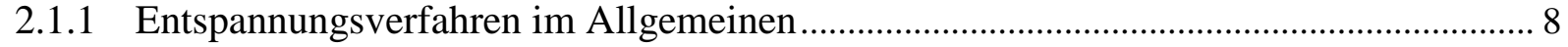

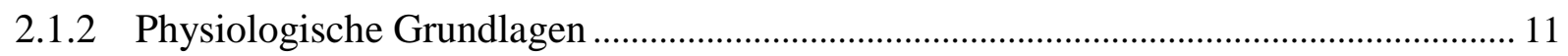

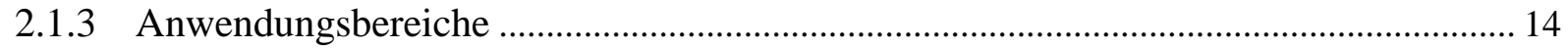

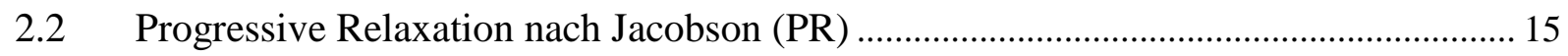

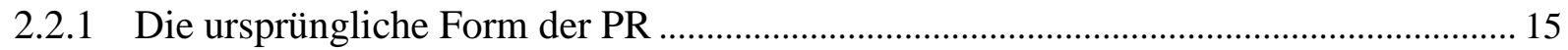

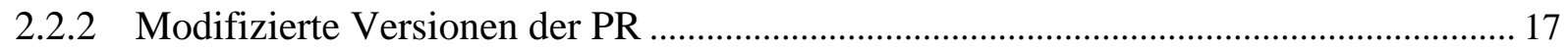

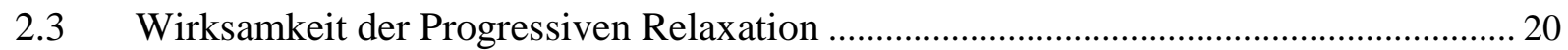

2.4 Anwendungsbereiche der Progressiven Relaxation ....................................................... 26

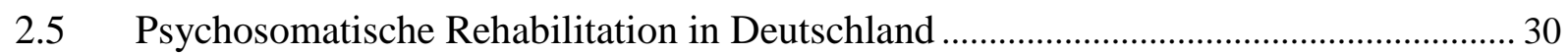

2.5.1 Entspannungsverfahren als Bestandteil psychosomatischer Rehabilitation ................. 31

2.5.2 Progressive Relaxation in der Fachklinik für Psychosomatische Rehabilitation und Psychotherapie der MediClin Bliestal Kliniken, Blieskastel ........................................... 33

2.6 Fragestellung und Zielsetzung der Dissertation.............................................................. 36

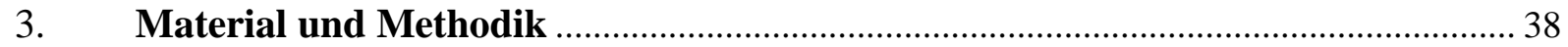

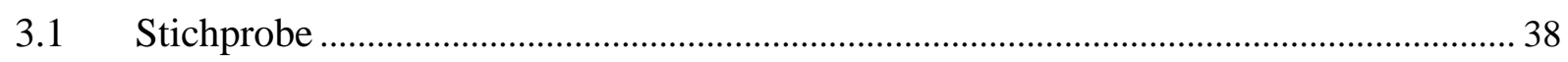

3.2 Aufbau und zeitlicher Rahmen der Untersuchung …..................................................... 40

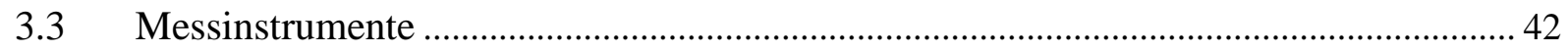

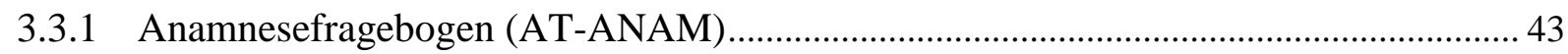

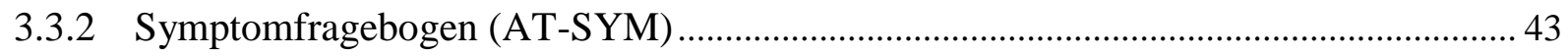

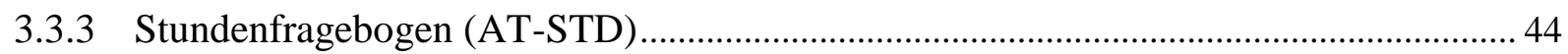

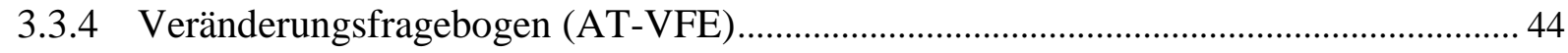




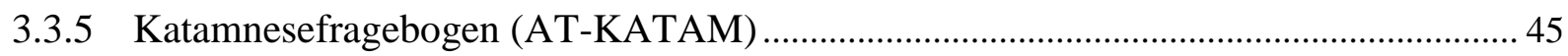

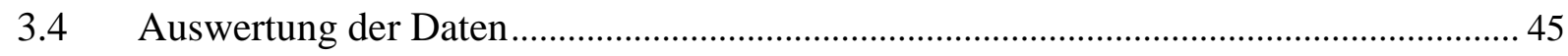

4. Ergebnisse

4.1 Übungshäufigkeit während des Klinikaufenthalts außerhalb des PR-Kurses................ 48

4.1.1 Übungshäufigkeit im Rahmen des modifizierten PR-Kurses ........................................ 48

4.1.2 Vergleich mit Ergebnissen der Vorläuferstudie............................................................ 50

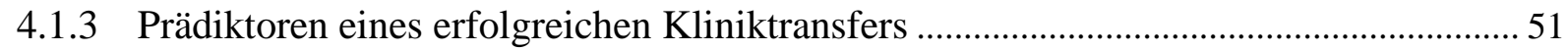

4.2. Übungshäufigkeit im Alltag nach dem Klinikaufenthalt.............................................. 52

4.2.1 Erfolgreicher Alltagstransfer im Rahmen des modifizierten PR-Kurses ....................... 52

4.2.2 Vergleich mit Ergebnissen der Vorläuferstudie .................................................................. 53

4.3 Prädiktoren eines erfolgreichen Alltagstransfers …......................................................... 54

4.4 Zusammenhang zwischen der Übungshäufigkeit im Alltag und anspannungsbezogenen

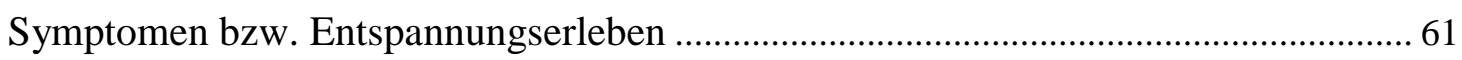

4.4.1 Symptome und Symptomentwicklung im Patientenkollektiv allgemein ....................... 61

4.4.2 Symptome, Symptomentwicklung und verändertes Entspannungserleben im Vergleich

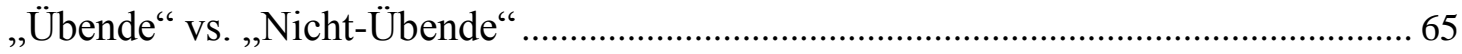

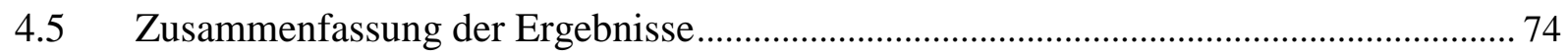

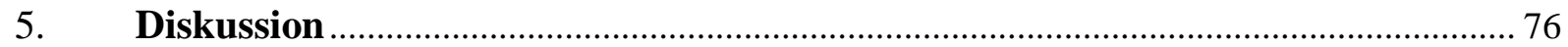

5.1 Selbstständiges Üben während des Klinikaufenthalts und im Alltag ............................ 76

5.2 Prädiktoren eines erfolgreichen Alltagstransfers von PR .............................................. 82

5.3 Zusammenhang zwischen der Übungshäufigkeit im Alltag und der Symptombelastung

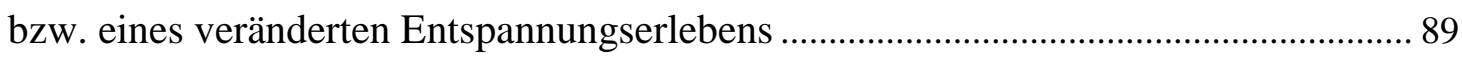

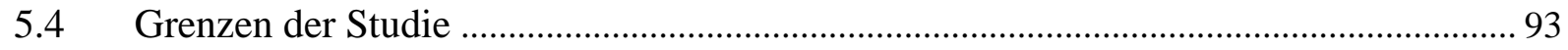

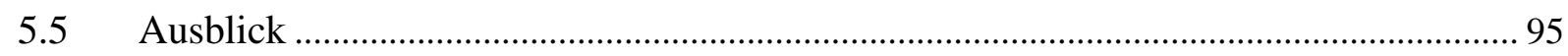

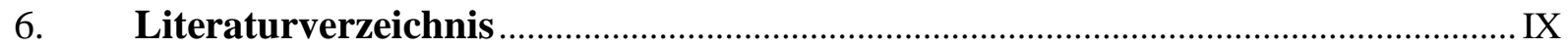

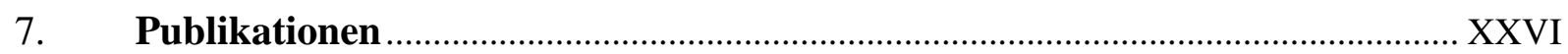

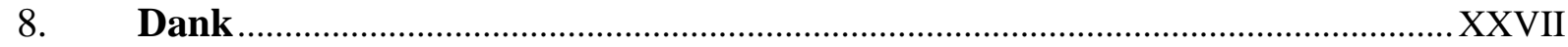

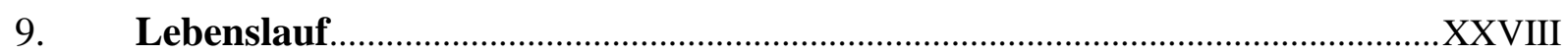

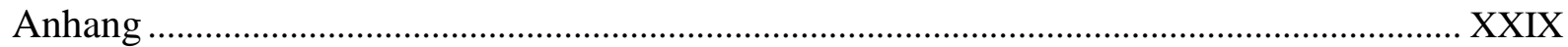




\section{Abkürzungsverzeichnis}

AT:

AT-ANAM:

AT-KATAM:

AT-STD:

AT-SYM:

AT-VFE:

Beta:

ES:

ETM:

GI:

ICD-10:

MW:

$\mathrm{N}$ :

n. s.:

p:

PR:

r:

$\mathrm{R}:$

$\mathrm{R}^{2}$ :

SD:

VDR:
Autogenes Training

Anamnesefragebogen

Katamnesefragebogen

Stundenfragebogen

Symptomfragebogen

Veränderungsfragebogen

Standardisierter Regressionskoeffizient

Effektstärke

Evidenzbasierte Therapiemodule

Gelenkte Imagination

International Statistical Classification of Diseases and Related Health

Problems, Internationale statistische Klassifikation der Krankheiten und verwandter Gesundheitsprobleme (10. Revision)

Mittelwert

Anzahl der Probanden

nicht signifikant

p-Wert, Signifikanz

Progressive Relaxation

Korrelationskoeffizient

Multipler Korrelationskoeffizient

Bestimmtheitsmaß

Standardabweichung

Verband Deutscher Rentenversicherungsträger 


\section{Abbildungsverzeichnis}

Abb. 1: Aufbau des Entspannungskurses der Psychosomatischen Fachklinik der MediClin Bliestal Kliniken in Blieskastel

Abb. 2: $\quad$ Übersicht über Patientenzahlen und Drop out-Analyse

Abb. 3: Häufigkeitsangaben zum selbstständigen Üben zu T2

Abb. 4: $\quad$ Motivation zum selbstständigen Üben durch den Kursleiter

Abb. 5: Gelegenheit zum Austausch von eigenen Erfahrungen beim selbstständigen Üben

Abb. 6: Häufigkeitsangaben zum Üben im Alltag (T3)

Abb. 7: $\quad$ Bewertung der Gruppengröße auf bipolarer Skala

Abb. 8: $\quad$ Bewertung der Gruppengröße auf unipolarer Skala

Abb. 9: $\quad$ Benotung des Entspannungskurses

Abb.10: $\quad$ Bewertung des aktuellen persönlichen Wohlbefindens zu T3

Abb.11: Übersicht über Studienteilnehmer und Drop-out-Quoten der beiden zu vergleichenden Studien 


\section{Tabellenverzeichnis}

Tab. 1: Anwendungsfelder von Entspannungsverfahren nach Petermann u. Vaitl (2009)

Tab. 2: $\quad$ Angewandte Entspannung nach Öst

Tab. 3: $\quad$ Studien zur Wirksamkeit bei verschiedenen Schmerzformen und deren Ergebnisse

Tab. 4: Studien zur Wirksamkeit bei somatischen Erkrankungen und deren Ergebnisse

Tab. 5: $\quad$ Anwendungsgebiete der Progressiven Relaxation (Ohm, 2004)

Tab. 6: Anwendungsbereiche von PR bei psychischen Störungen nach Krampen (2004)

Tab. 7: $\quad$ Absolute und relative Kontraindikationen der PR nach Ohm (2004)

Tab. 8: Allgemeine und spezielle Indikationen stationärer psychosomatischer Rehabilitation

Tab. 9: $\quad$ Übersicht Messzeitpunkte, eingesetzte Messinstrumente und Anzahl der Probanden (N)

Tab.10: $\quad$ Gruppen innerhalb des Patientenkollektivs, definierte Übungshäufigkeit und Anzahl der Probanden (N)

Tab.11: $\quad$ Vergleich der beiden Studien hinsichtlich Übungshäufigkeit in der Klinik

Tab.12: $\quad$ Mögliche Prädiktoren eines erfolgreichen Kliniktransfers

Tab.13: $\quad$ Vergleich der beiden Studien hinsichtlich Übungshäufigkeit im Alltag

Tab.14: $\quad$ Mögliche Prädiktoren eines erfolgreichen Alltagstransfers

Tab.15: $\quad$ Übersicht der Mittelwerte der Skalen des AT-VFE zu T2 und T3

Tab.16: Bewertung des Inhalts des Grundlagenseminars

Tab.17: $\quad$ Übersicht über die Ergebnisse der linearen Regressionsanalyse

Tab.18: $\quad$ Mittelwertvergleiche der Skalen des AT-SYM, Veränderungen zwischen T1, T2 und T3 und Effektstärken

Tab.19: Korrelation der Übungshäufigkeit im Alltag mit anspannungsbezogenen Symptomen zu den drei Messzeitpunkten T1, T2, T3

Tab.20: $\quad$ Korrelation der Symptomreduktion zwischen den Messzeitpunkten mit der Übungshäufigkeit im Alltag

Tab.21: Mittelwertvergleich der Skalen des AT-SYM zwischen „Übenden“ und „NichtÜbenden“ zu den verschiedenen Messzeitpunkten 
Tab.22: $\quad$ Mittelwertvergleich (T1, T2), Vergleich der Differenzwerte (T1-T2) und des veränderten Entspannungserlebens zu T2 zwischen „Übenden“ und „NichtÜbenden“"

Tab.23: Vergleich „Übende“ vs. „Nicht-Übende“ hinsichtlich Symptomreduktion (T1T3, T2-T3) und veränderten Entspannungserlebens zu T3

Tab.24: $\quad$ Vergleich der extremen Quartile hinsichtlich anspannungsbezogener Symptome zu T3, deren Entwicklung (T1-T3, T2-T3) und veränderten Entspannungserlebens zu T3

Tab.25: Vergleich der „häufig Übenden“ mit den „regelmäßig Übenden“ hinsichtlich anspannungsbezogener Symptome zu T3, deren Entwicklung (T1-T3, T2-T3) und veränderten Entspannungserlebens zu T3

Tab.26: Vergleich der „Nicht-Übenden“ mit den „selten Übenden“ hinsichtlich anspannungsbezogener Symptome zu T3, deren Entwicklung (T1-T3, T2-T3) und veränderten Entspannungserlebens zu T3 


\section{Zusammenfassung}

\section{Verbesserung des Transfers von Progressiver Relaxation in den Alltag der Patienten nach stationärer psychosomatischer Rehabilitation}

Hintergrund: Entspannungsverfahren gehören als Evidenzbasierte Therapiemodule (ETM) zu den Reha-Therapiestandards der Deutschen Rentenversicherung. Dabei stellt die Progressive Relaxation nach Jacobson (PR) eine der am häufigsten eingesetzten Entspannungstechniken in deutschen Rehabilitationskliniken dar. Obwohl sich bei derartigen Programmen rasche Übungserfolge einstellen, scheitert ein erheblicher Anteil der Patienten am Transfer des Gelernten in den Alltag. Das Ziel der vorliegenden Studie war daher die Evaluation des modifizierten Entspannungskurses (im Vergleich zu einer Pilotstudie aus dem Jahre 2006) sowie die Identifikation möglicher Prädiktoren eines erfolgreichen Klinik- und Alltagstransfers. Darüber hinaus wurde die Entwicklung anspannungsbezogener Beschwerden im Zusammenhang mit der Übungshäufigkeit untersucht.

Methodik: Bei der vorliegenden Studie handelt es sich um eine prospektive Längsschnittstudie. Die Probanden nahmen im Rahmen eines vier- bis sechswöchigen Reha-Aufenthalts an einem sechs Übungsstunden umfassenden PR-Kurs teil und wurden zu drei verschiedenen Zeitpunkten mittels einer modifizierten Version des ,diagnostischen und evaluativen Instrumentariums für Entspannungstraining und Entspannungstherapie (ET-EVA)“ nach Krampen (2002a) befragt: bei Ankunft (T1), bei Abreise (T2) und drei Monate nach Abreise postalisch (T3). Von 447 stationär aufgenommen Patienten mit Indikation zur Teilnahme am PR-Kurs erklärten sich 411 Patienten (91,9\%) zur Teilnahme an der Studie bereit (73,7\% weiblich, MW(Alter)=49,14 Jahre, SD=8,48). Zu T2 verblieben 350 Patienten (85,2\%) und zu T3 schickten 274 Patienten die Fragebögen zurück, was einer Rücklaufquote von 78,3\% entspricht. Eine Übungshäufigkeit von mindestens einmal pro Woche wurde als erfolgreich definiert. Die Ergebnisse hinsichtlich Klinik- und Alltagstransfer wurden mit denen einer Pilotstudie aus dem Jahre 2006 verglichen. Als Maß der Veränderung der Symptome wurden Differenzwerte der Skalenmittelwerte zwischen den Messzeitpunkten und Effektstärken berechnet. Mittels bivariater Korrelationen und einer linearen Regressionsanalyse wurden Prädiktoren der Übungshäufigkeit im Alltag ermittelt.

Ergebnisse: 69,4\% der Patienten übten bereits während des Klinikaufenthalts mindestens einmal pro Woche. Dem veränderten Entspannungserleben nach dem Kurs konnte ein - aller- 
dings geringer - positiver Einfluss auf den Kliniktransfer zugeschrieben werden $(r=-0,184$; p <0,01). Im Vergleich zur Pilotstudie aus dem Jahre 2006 konnte nach konservativer Schätzung ein signifikant höherer Anteil der Studienteilnehmer die erlernten PR-Übungen in den Klinikalltag integrieren.

Zu T3 konnten 50,4\% der Studienteilnehmer die Entspannungsübungen im Alltag erfolgreich umsetzen. Weder nach optimistischer noch nach konservativer Schätzung unterschieden sich diesbezüglich die Erfolgsquoten der beiden zu vergleichenden Studien.

Die Übungshäufigkeit während des Klinikaufenthalts $(r=0,558 ; \mathrm{p}<0,01)$ und das veränderte Entspannungserleben nach drei Monaten $(r=-0,356 ; \mathrm{p}<0,01)$ konnten als Prädiktoren eines erfolgreichen Alltagstransfers identifiziert werden. Desweiteren fanden sich schwach ausgeprägte Korrelationen zwischen der Übungshäufigkeit im Alltag und dem veränderten Entspannungserleben bei Abreise $(r=-0,240 ; p<0,01)$, der Benotung des Entspannungskurses $(\mathrm{r}=+0,243 ; \mathrm{p}<0,01)$ sowie dem aktuellen subjektiven Wohlbefinden zu T3 ( $r=-0,165 ; \mathrm{p}<0,01)$. Das Alter der Patienten korrelierte nur schwach mit dem Alltagstransfer $(r=-0,127 ; \mathrm{p}<0,05)$. Demnach war der erfolgreiche Alltagstransfer bei jenen Patienten wahrscheinlicher, die erfolgreich bereits während des Klinikaufenthalts übten, eine starke Veränderung ihres Entspannungserlebens und Wohlbefindens (zu T2 und vor allem zu T3) erfuhren, den Entspannungskurs positiv bewerteten und eine geringe Symptombelastung im Alltag aufwiesen. Weder die Bewertung der Gruppengröße noch das Geschlecht hatten einen Einfluss auf die Übungshäufigkeit im Alltag. Im Rahmen der linearen Regressionsanalyse blieb der Effekt der „Übungshäufigkeit während des Klinikaufenthalts außerhalb der Kursstunden“ (Beta=0,506; $\mathrm{p}<0,01)$ und des ,veränderten Entspannungserlebens zu T3“ (Beta=-0,275; p<0,01) signifikant. Die multiple Korrelation betrug $\mathrm{R}=0,640$, d. h. 40,9\% der Varianzen im Kriterium können durch die Prädiktoren erklärt werden.

Das Entspannungserleben und Wohlbefinden zeigte bei Abreise (T2) eine positive Veränderung (MW der 12 Items zu T2=5,044; SD=0,98; N=349), die auch nach drei Monaten in abgeschwächter Form noch nachweisbar war (MW der 12 Items zu T3=4,79; SD=1,1; N=262). Die Beschwerden aller sechs Symptomskalen nahmen von T1 zu T2 signifikant ab. Die Symptombelastung nach drei Monaten blieb signifikant unter dem Ausgangsniveau von T1. Die größten Effektstärken konnten dabei innerhalb der Skalen der anspannungsbezogenen Beschwerden ermittelt werden $(\mathrm{ES}=0,85$ bis $\mathrm{ES}=1,63)$. Es zeigten sich schwache Korrelationen zwischen der Übungshäufigkeit im Alltag und der Belastung durch anspannungsbezogene Symptome zu T3 ( $r=0,199 ; \mathrm{p}<0,01)$ sowie der Entwicklung anspannungsbezogener Beschwerden - besonders im Verlauf von T1 zu T3 (r=-0,245; p<0,01). Wenig 
belastete Patienten oder solche, die eine besonders ausgeprägte Verbesserung ihrer Beschwerden erfuhren, neigten dazu, PR im Alltag etwas häufiger einzusetzen.

Patienten, die mindestens einmal pro Woche übten, gaben im Vergleich zu den nicht übenden Probanden signifikant weniger anspannungsbezogene Beschwerden zu T3 an, zeigten eine signifikant stärkere Abnahme der Beschwerden von T1 zu T3 und konnten eine signifikant stärkere Veränderung des Wohlbefindens und Entspannungserlebens zu T2 und T3 erzielen. Patienten mit einer Übungshäufigkeit von mindestens einmal täglich unterschieden sich von jenen, die ein- bis mehrmals pro Woche übten, lediglich hinsichtlich der Symptomentwicklung auf der Skala „körperliche und psychische Erschöpfung“ von T1 zu T3.

Schlussfolgerung: Die Progressive Relaxation hat als wirksames und nachhaltiges Verfahren zu Recht einen festen Platz im Therapiekonzept vieler Rehabilitationskliniken. Dabei kommt der gefühlten Veränderung im Sinne der Selbstwirksamkeit eine besondere Bedeutung zu. Das initiale Erleben von Entspannung sollte im Rahmen zukünftiger Kurse etwa durch Schaffung geeigneter Rahmenbedingungen erleichtert und erlebbar gemacht werden.

Das Entfalten der positiven Auswirkungen von PR erfordert Zeit und kontinuierliches Üben. Die Unterschiede zwischen übenden und nicht-übenden Patienten waren daher vor allem im Rahmen der katamnestischen Befragung signifikant. Dabei zeigte sich eine Übungshäufigkeit von mindestens einmal wöchentlich als wirkungsvoll und effektiv. Die tägliche Übungsroutine erzielte bezüglich Symptombelastung keinen zusätzlichen Benefit. Darüber hinaus ist die regelmäßige Übungsroutine vom aktuellen Wohlbefinden und Beschwerdebild abhängig und wird vermehrt in Zeiten geringer Symptombelastung umgesetzt. Die präventiven und kurativen Wirkungen der Progressiven Relaxation können sich jedoch erst entfalten, wenn auch in Zeiten vermehrter Symptombelastung geübt und von den Effekten profitiert wird. Zukünftige Kursteilnehmer sollten darauf hingewiesen und zur Anwendung auch im hektischen Alltag motiviert werden.

Untersuchungen im ambulanten Bereich oder in Fachrichtungen mit unterschiedlichem Patientenkollektiv als auch der Einfluss auditorischer Hilfsmittel können interessante Ansatzpunkte zukünftiger Forschungsarbeiten sein. 


\section{Summary}

\section{The improvement of the transfer into daily life of progressive relaxa- tion after an inpatient psychosomatic rehabilitation}

Background: Relaxation techniques as evidence based therapy modules are part of the rehabilitation therapy standards of the German pension fund. Thereby, progressive relaxation (PR) is one of the most frequently applied relaxation programs in German psychosomatic rehabilitation clinics. Although the clinical efficiency is encouraging, the transfer into daily life often fails. Therefore, the aim of this prospective study was the evaluation of the modified relaxation course (compared to a pilot study of 2006) and the identification of predictors of a successful transfer into clinical and daily life. Furthermore, the development of tensionrelated symptoms dependent on the frequency of continuous practise was detected.

Methods: The current study is a longitudinal section study. The patients attended a six-hourcourse of progressive relaxation and were interviewed at three different times by a modified version of a relaxation-specifique questionnaire ("Diagnostisches und Evaluatives Instrumentarium für Entspannungstraining und Entspannungstherapie (ET-EVA)" by Krampen (2002a)). The questionnaire was given at the beginning of therapy (T1), at discharge (T2) and three months after discharge by postal service (T3). 411 of 447 patients (91.9\%) with medical indication of a PR course participated in this study (73.7\% females, $\mathrm{M}($ age $)=49.14, \mathrm{SD}=8.48)$. At discharge, 350 patients $(85.2 \%)$ remained. After three months 274 patients sent the completed questionnaires back - the response rate was $78.3 \%$. A frequency of exercising at least once a week was defined as successful. The results - regarding the transfer into clinical and daily life - were compared with the results of a pilot study of 2006. To detect the extent of symptom improvement difference values between the different measuring times and effect sizes were calculated. To identify predictors of the frequency of daily practise bivariate correlations and linear regression was used.

Results: $69.4 \%$ of the patients were able to continue the exercises successfully beyond the course. The improved experience of relaxation after the program had a positive influence on the frequency of practising during hospital stay $(r=-0.184 ; \mathrm{p}<0.01)$. According to conservative appraisal the success rate of 2010 - regarding the successful transfer during hospital stay was significantly higher than within the pilot study of 2006. Three months after discharge $50.4 \%$ of the participants were practising at least once a week on their own. There was no 
difference between the pilot and the current study (according neither to conservative nor to optimistic appraisal).

The frequency of practise during hospital stay $(\mathrm{r}=0.558 ; \mathrm{p}<0.01)$ and the changed experience of relaxation after three months $(\mathrm{r}=-0.356 ; \mathrm{p}<0.01)$ could be identified as predictors of a successful transfer into daily life of progressive relaxation. Furthermore, there were small correlations between the transfer of PR and the changed experience of relaxation at discharge $(\mathrm{r}=-0.240 ; \mathrm{p}<0.01)$, the grading of the relaxation training $(\mathrm{r}=0.243 ; \mathrm{p}<0.01)$, the age of the patients $(\mathrm{r}=-0.127 ; \mathrm{p}<0.05)$ and the current subjective wellbeing at $\mathrm{T} 3(\mathrm{r}=-0.165 ; \mathrm{p}<0.01)$. Neither the gender nor the grading of the group size had an influence on the frequency of practising in the daily routine. Linear regression analysis showed that the effect of the frequency of practise during hospital stay $(\operatorname{Beta}=0.506 ; \mathrm{p}<0.01)$ and the changed experience of relaxation after three months (Beta $=-0.275 ; \mathrm{p}<0.01)$ remained significant. The multiple correlation was $\mathrm{R}=0.640$, i.e. $40.9 \%$ of the variances within the criteria can be explained by the predictors.

At the point of discharge the experience of relaxation was positively changed (mean of the 12 items at $\mathrm{T} 2=5.044 ; \mathrm{SD}=0.98 ; \mathrm{N}=349$ ) and after three months still low positive (mean of the 12 items at $\mathrm{T} 3=4.79 ; \mathrm{SD}=1.1 ; \mathrm{N}=262$ ). The items of all six symptom scales decreased significantly from $\mathrm{T} 1$ to $\mathrm{T} 2$. The feeling of discomfort after three months was significantly below the base level of T1. Especially within the scales of tension-related symptoms large effect sizes were measured $(\mathrm{ES}=0.85$ up to $\mathrm{ES}=1.63$ ). There were small but significant correlations between the frequency of daily practise and the tension-related symptoms at T3 ( $r=0.199$; $\mathrm{p}<0.01)$ as well as the development of tension-related symptoms - especially from $\mathrm{T} 1$ up to T3 ( $\mathrm{r}=-0.245 ; \mathrm{p}<0.01)$. Patients with less symptoms or a strong improvement of wellbeing tended to practise more often.

The patients who practised at least once a week - compared to the not-practising patients declared significantly less tension-related symptoms at T3, showed a significantly higher decrease of symptoms from T1 up to T3 and could achieve a significantly stronger change of wellbeing and relaxation experience at $\mathrm{T} 2$ and $\mathrm{T} 3$. The patients with a frequency of practising at least once a day differ from the patients that practised once to several times a week only in symptom development on the scale "somatic and mental depletion" from T1 up to T3.

Conclusion: The progressive relaxation as an effective and lasting technique is rightfully a part of the therapy concept of many rehabilitation clinics. Thereby, the experienced alteration in terms of self-efficacy plays a meaningful role. The initial experience of relaxation should be simplified and more sensible in future courses - for example by creating more pleasant 
conditions. It needs time and continuous practise to benefit. Therefore, the differences between practising patients and not-practising patients were significant especially within the follow-up. The frequency of practise once a week turned out to be the most effective. A daily routine showed no greater benefit concerning symptoms. Furthermore, a daily routine of practise depends on severity of symptoms and status of wellbeing and is rather performed in stress-free times. But preventive and curative effects of progressive relaxation can only fully unfold if the exercises are also performed in more busy times. Future participants should be informed about these facts and motivated to practise also in a hectic daily routine.

Interesting approaches for future studies might be both the acquisition of progressive relaxation effects under ambulant conditions or in other disciplines with different patient population and the influence of auditory supplies. 


\section{Einleitung}

Die Progressive Relaxation stellt eines der am häufigsten eingesetzten Verfahren in deutschen Rehabilitationskliniken dar (Brenner, 2002). Gründe dafür sind sowohl die einfache Erlernbarkeit dieses Verfahrens, die universelle Anwendbarkeit als auch die rasche Wirkungsentfaltung (Olschewski, 1996). Progressive Relaxation kann wörtlich als ,voranschreitende Entspannung“ (Ohm, 2007) übersetzt werden, was bereits das systematische Vorgehen bei dieser Methode verdeutlicht. Durch Anspannung und nachfolgende Entspannung einzelner Muskelgruppen kann ein umfassender Ruhezustand erreicht werden.

Im Laufe der Zeit entwickelten sich aus der ursprünglichen ausführlichen Form der Übungsmethodik von Jacobson (1929) modifizierte und gekürzte Versionen, wodurch dieses Verfahren zunehmend an Alltagstauglichkeit gewinnen konnte. Im Rahmen von Gruppenentspannungsprogrammen in Rehabilitationskliniken lassen sich rasche Übungserfolge erzielen. Dennoch scheitert ein nicht zu vernachlässigender Anteil der Patienten am Transfer des Gelernten in den Alltag (Derra, 2007). Interessant ist in diesem Zusammenhang die Frage, durch welche Faktoren die Aufrechterhaltung des Übungsverhaltens bei den übrigen Patienten bedingt wird. Bereits bekannt ist, dass Vorerfahrungen und Teilnahmemotive (Krampen, 2002b) als auch die Übungshäufigkeit während des Klinikaufenthalts (Bernardy et al., 2008) den Therapieerfolg und Alltagstransfer von Progressiver Relaxation positiv beeinflussen.

Ziel der vorliegenden Arbeit ist es, Prädiktoren eines erfolgreichen Klinik- und Alltagstransfers zu identifizieren und den Einfluss einer möglichen Symptomreduktion auf die Übungshäufigkeit im Alltag zu erfassen. Darüber hinaus soll untersucht werden, inwieweit die Symptomentwicklung an sich von der Übungshäufigkeit beeinflusst wird. Weitere Ziele der vorliegenden Studie sind die Untersuchung einer möglichen Verbesserung des Alltagstransfers nach Modifikation des damaligen Entspannungstrainings sowie die Ermöglichung einer repräsentativen Stichprobe bei genauer Drop-out-Analyse. Zudem werden die Ergebnisse hinsichtlich Klinik- und Alltagstransfer mit denen der Pilotstudie von Arndt aus dem Jahre 2005/2006 verglichen. 


\subsection{Wirkungsweise und Indikationen von Entspannungsverfahren}

\subsubsection{Entspannungsverfahren im Allgemeinen}

Entspannung spielt eine lebenswichtige Rolle im menschlichen Organismus. Nur durch die Balance zwischen Anspannung und Aktivität auf der einen und Entspannung und Passivität auf der anderen Seite kann die Funktionsfähigkeit eines Organismus gewährleistet werden. Allen „kulturellen“ Entspannungsverfahren gemein ist die zentrale Rolle der Hedonie, also des Wohlbefindens im eigentlichen Sinne. Diesen stehen die Methoden aus dem wissenschaftlichen und klinischen Bereich gegenüber, die sich mit interozeptiven, vegetativen und neurophysiologischen Entspannungsprozessen beschäftigen, zur Therapie psychischer Störungen entwickelt wurden und Bestandteile sowohl von Präventions-, als auch Therapieund Rehabilitationsprogrammen sind (Ruhl et al, 2006). Die klinischen Entspannungstechniken sind in den vergangenen drei Jahrzehnten zudem Gegenstand vielfältiger Forschung geworden, um sowohl physiologisches Fundament als auch klinischen Nutzen dieser Techniken zu belegen. Studien zur differentiellen Wirksamkeit führten zwar zu einem eingeschränkten, dafür aber deutlich präzisierten Wirkungsspektrum (Petermann und Vaitl, 2009).

Entspannungsverfahren sind nach den Richtlinien des Bundesausschuss der Ärzte und Krankenkassen über die Durchführung der Psychotherapie (1998) sowohl Bestandteil psychotherapeutischer Anwendungsformen als auch Methoden psychosomatischer Grundversorgung. Im Verlauf der Verbreitung dieser Therapiemodule haben sich Sektionen innerhalb ärztlicher und psychologischer Fachgesellschaften gebildet, die sich im Rahmen von Konsensuskonferenzen um einheitliche Standards zur Durchführung, Indikation und Erfolgskriterien von Entspannungstechniken bemühten.

Nach Smith (1988) stellt die ,arousal reduction“, also die Aktivitätsminderung psychophysiologischer Vorgänge, die Hauptwirkung von Entspannungsverfahren dar. Neben diesen körperlichen Effekten besitzen Entspannungstechniken ein oft vernachlässigtes psychotropes Potenzial - nämlich das der kognitiven Restrukturierung. Dieses Phänomen besteht aus der Sensibilisierung für und Fokussierung auf körperliche Vorgänge, dem Akzeptieren ungewohnter und neuartiger Empfindungen als eigen und dem Erwerb von Bewältigungsstrategien zum veränderten Umgang mit eigenen Problemen. Entspannung impliziert somit nicht nur den reinen hedonischen Genuss ohne Zutun, sondern erfordert Mitarbeit und Energie. 
Zum klinischen Standard-Repertoire der Entspannungsverfahren gehören (Vaitl und Petermann, 2000):

- Hypnose

- Autogenes Training

- Meditation

- Imaginative Verfahren

- Progressive Relaxation

- Biofeedback

Hypnose. Den Ursprung aller Entspannungsverfahren stellt die Hypnose dar. Viele alte Hochkulturen praktizierten hypnotische Techniken - lange bevor der allgemeine Begriff der Hypnose im 19. Jahrhundert geprägt wurde. Um 1840 definierte James Braid den „nervösen Schlaf" als Hypnose (Braid, 1846). Hippolyte Bernheim bezeichnete dieses Verfahren im 19.Jahrhundert erstmals als „Psychotherapie“ (Bernheim, 1891, 1892), während Jean-Martin Charcot Hypnose als Therapie bei hysterischen Patienten in einen pathologischen Kontext stellte (Möller et al., 2005; Charcot, 1892-94). Im deutschsprachigen Raum wurde die Hypnose vor allem durch Oskar Vogt und seinen Schüler Johannes Heinrich Schultz weiterentwickelt (Schultz, 1924, 1934).

Autogenes Training. Schultz führte die in seinen Hypnose-Sitzungen beobachteten selbsterzeugten (,autogenen“), psychovegetativen Umschaltprozesse auf Selbstsuggestionen zurück (Schultz, 1969, 1970). „Training“ impliziert dabei die Notwendigkeit des Übens. Die drei Hauptprinzipien umfassen kognitive Fokussierung auf körperliche Vorgänge (,konzentrative Selbstentspannung“; Schultz, 1956, 1973), mentale Wiederholung bestimmter Selbstinstruktionen und Dämpfung intero- und exterozeptiver Stimulation (Vaitl und Petermann, 2000).

Meditation. Die Meditation stellt durch die Heterogenität der Methoden und ihre Wurzeln im religiösen Bereich einen Sonderfall dar (Linden, 2000; Ott, 2009). Ziele der klassischen Meditation sind eine meditative Versenkung in das eigene Innere (Maharashi, 1975), die Erweiterung von Bewusstsein und tiefer innerer Ruhe und spirituelles Wachstum (Brenner, 2004). Entspannung und Stressbewältigung sind dabei weniger primäres Ziel als vielmehr notwendige Voraussetzung der gewünschten Bewusstseinsveränderungen (Linden, 2000). Das Ziel der jüngeren Meditationstechniken kann mit einer Steigerung der Selbstwirksamkeit beschrieben werden (Bandura, 1977). Die Anerkennung der Meditation als wissenschaftlich fundierte Entspannungstechnik gelang Wallace (1970) mit Veröffentlichung seiner Ergebnisse in der Zeitschrift „Science“. 
Imagination. Ein Verfahren, das auch bereits um 1900 Anwendung fand, ist das der Imagination (Betts, 1909). Darunter versteht man psychophysiologische Prozesse, bei denen ohne reale Reize von außen realitätsnahe Wahrnehmungen jeglicher Sinnesqualitäten auf der Vorstellungsebene erzeugt werden (Petermann und Vaitl, 2009; Kirn et al., 2009). „Imagery Rescripting“ (Vorstellungssubstitution) und „Guided Imagery“ (Gelenkte Imagination) stellen einige Beispiele verhaltenstherapeutisch-fundierter Verfahren dar, die heutzutage vor allem im Rahmen von Selbsthilfeprogrammen Anwendung finden (Tindle et al., 2006).

Biofeedback. Physiologische Prozesse und deren Biosignale bzw. Messgrößen, die in der Regel unserer Wahrnehmung nicht zugänglich sind, werden in akustische oder optische Signale umgewandelt und so dem Bewusstsein zugänglich und willentlich beeinflussbar gemacht. Vormals autonome Körperfunktionen können auf diese Art gesteuert und reguliert werden (Schwartz und Andrasik, 2005). Selbstkontrolle wird somit direkt spür- und objektivierbar. Domänen des Biofeedbacks sind Erfassung von Muskelaktivitäten (EMG-Feedback), Messung von Temperaturveränderungen (vasomotorisches Feedback) und Registrierung von Herzfrequenz- und Blutdruck-Schwankungen im Rahmen des kardiovaskulären Biofeedbacks.

Gemeinsamkeiten. Entspannungsverfahren sind in der Lage, ihre Wirkungen je nach Angriffspunkt rasch zu entfalten. Dabei ist allen Verfahren die Notwendigkeit des kontinuierlichen Übens gemein. Eine weitere Gemeinsamkeit liegt im somatotropen und psychotropen Potenzial. So können sowohl körperliche als auch langfristig psychische Veränderungen im Sinne der kognitiven Umstrukturierung erreicht werden. Eigenkompetenz und Selbstkontrolle sind zentrale Ziele der Verhaltenstherapie, bei denen Entspannungsverfahren unterstützende Hilfestellung bieten können (Petermann und Vaitl, 2009). Die Schulung von Konzentration und gerichteter Aufmerksamkeit bei gleichzeitig beruhigender Wirkung sind weitere charakteristische Merkmale. Die Übenden lernen, störende Außenreize zu ignorieren, sich auf das innere Erleben zu konzentrieren und eigene Bedürfnisse verstärkt wahrzunehmen. Dabei spielt die Imagination als Vermittlungsinstanz zwischen innerer und äußerer Welt eine zentrale Rolle (Vaitl und Petermann, 2000).

Alle Entspannungsverfahren leisten einen entscheidenden Beitrag zum Wohlbefinden des Übenden. Selbstvertrauen, Konzentrationsvermögen und innere Ruhe tragen zu einer ausgeprägten Therapiemotivation bei, die sich auch positiv auf die Erfolgsaussichten anderer Therapiemodule auswirken kann. Aus diesen vielfältigen Gründen haben sich Entspannungsverfahren zu einem integralen Bestandteil eines multimodalen Behandlungskonzepts entwickelt (Petermann und Vaitl, 2009). 


\subsubsection{Physiologische Grundlagen}

Da Entspannungsverfahren es ermöglichen, Stressreaktionen aufzuheben oder abzumildern, sollen daher kurz einige Stressmodelle beleuchtet werden, bevor auf die körperlichen Vorgänge während der Entspannungsreaktion eingegangen wird. Cannon (1914) beschrieb bereits zu Beginn des 20. Jahrhunderts physiologische Veränderungen des Körpers, um in Stresssituationen im Rahmen einer sogenannten „flight-or-fight-response“ (Davis et al., 2008) zu reagieren. Seyles „Syndrom des Krankseins“ (1984) beschrieb Stress als unspezifische Reaktion unseres Körpers auf jegliche Art von (körperlicher oder seelischer) Anforderung (Seyle, 1978, 1984). Sein „Allgemeines Adaptionssyndrom“ (1952) umfasst dabei drei Phasen: Die Alarmreaktion beinhaltet eine Sympathikusaktivierung mit Ausschüttung von Noradrenalin und Adrenalin aus dem Nebennierenmark. Folgen davon sind Pupillen- und Bronchiendilatation, Vasokonstriktion, Lipolyse, Glykogenolyse mit Mobilisierung von Glucosereserven, Zunahme von Blutdruck, Herzfrequenz und Muskelperfusion und Suppression sowohl von Speichelsekretion als auch gastrointestinaler und urogenitaler Peristaltik. In der Widerstandsphase vermag unser Körper die Balance zwischen Anforderungen und Ressourcen $\mathrm{zu}$ halten, wohingegen die Erschöpfungsphase durch Energiemangelzustände verbunden mit zunehmend geschwächter Immunabwehr - und somit der Manifestation stressbedingter Krankheiten - gekennzeichnet ist.

Die von Benson (1975) beschriebene „relaxation response“ beschreibt dagegen den entgegengesetzten Pol. Die Entspannungsreaktion als Bestandteil des natürlichen Verhaltensrepertoires eines Menschen kann unter bestimmten Lebens- und Umgebungsbedingungen automatisch eingeleitet werden (Derra, 2007). Physiologische Reaktionen sind ein Abfall des arteriellen Blutdrucks bei peripherer Vasodilatation (vor allem in den Hautarealen) mit Wärmeempfinden, Verlangsamung von Atem- und Herzfrequenz, Gleichmäßigkeit der Atemzyklen, Verminderung des Sauerstoffverbrauchs und des Muskeltonus, Abnahme von Hautleitfähigkeit, Spontanfluktuationen und Reflextätigkeit, Veränderungen der hirnelektrischen und neurovaskulären Aktivität und eine Zunahme der Magen-Darm-Peristaltik (Petermann und Vaitl, 2009).

Neuromuskuläre Veränderungen sind Angriffsziel der Progressiven Relaxation im Speziellen. Diese können mittels Elektromyogramm aufgezeichnet werden. Obwohl bei entspannten Muskelgruppen keine Aktionspotenziale mehr registriert werden können, ist ein gewisser Muskeltonus auch in entspannten Muskeln erhalten (Basmajian, 1963). Entspannungsverfahren erreichen somit lediglich eine Annäherung an das elektromyographische Null-Niveau in bestimmten Muskelpartien (Petermann und Vaitl, 2009). Aus dem neurophysiologischen In- 
stanzenmodell aus Motoneuronen, Muskelspindeln und supraspinalen Zentren ergibt sich, dass eine Entspannungsübung nicht nur auf bloße Ruhigstellung von Muskeln ausgelegt sein kann. Durch die Wahl gezielter Körperpositionen und geeigneter Umgebungsbedingungen sollen sowohl afferente Signale aus der Stützmotorik als auch störende Reize aus der Umgebung, die über eine Schreckreaktion die Formatio reticularis stimulieren können, reduziert werden (Petermann und Vaitl, 2009).

Kardiovaskuläre Veränderungen sind sowohl Ansatzpunkt als auch Ergebnis vieler Entspannungsverfahren. Dabei stehen periphere Vasodilatation und Blutdruckregulation im Fokus. Die Gefäßweite wird durch (alpha- und beta-) adrenerge Nervenendigungen in der glatten Gefäßmuskulatur, über vasoaktive Substanzen und lokale Faktoren wie die Blutgasverteilung reguliert. Durch Entspannungstechniken kann sowohl die adrenerge Aktivität gedämpft, als auch eine synergistische Wirkung bei Applikation vasoaktiver Substanzen und gleichzeitiger Anwendung einer Entspannungsübung erzielt werden. Dabei ist dieser Synergismus bei Anfängern weniger stark ausgeprägt als bei Geübten (Diehl, 1987). Die Empfindlichkeit der alpha- und beta-Rezeptoren und damit auch der lokale Blutfluss ist abhängig von der Umgebungstemperatur. Das Empfinden von Wärmesensationen - vorwiegend Ausdruck einer zunehmenden Sympathikolyse - kann somit durch die Wahl geeigneter Zimmertemperaturen $\left(30-36^{\circ} \mathrm{C}\right)$ erleichtert werden (Petermann und Vaitl, 2009). Während der Tastsinn im Rahmen einer Entspannungsübung eher herabreguliert wird, kann der „geschärfte“ Temperatursinn als Gradmesser für einen fortschreitenden Entspannungsprozess fungieren. Die genauen Mechanismen der antihypertensiven Wirkung verschiedener Entspannungstechniken, die durch zahlreiche Studien belegt werden konnte, bleiben jedoch bisher ungeklärt. Der verminderten Sympathikusaktivität wird auch diesbezüglich die Hauptwirkung zugeschrieben (Petermann und Vaitl, 2009). Dabei kommt abermals dem Training eine effektstabilisierende Bedeutung zu.

Entspannungstechniken haben neben den neuromuskulären und kardiovaskulären Effekten Auswirkungen sowohl auf die Atmung als auch auf elektrodermale und hirnelektrische Prozesse. Zusammenfassend lässt sich eine dämpfende Wirkung auf Atmungsparameter feststellen: Die Atemfrequenz verlangsamt sich, der Sauerstoffverbrauch nimmt ab, die Atemzyklen werden gleichmäßiger (Möller et al., 2005). Innerhalb eines Atemzyklus verlängert sich die Inspiration gegenüber der Exspiration - die Atmung wird flacher (Luthe, 1969). Es kommt zu einer Zunahme der abdominellen gegenüber der thorakalen Atmung (Petermann und Vaitl, 2009). Während diese Effekte im Allgemeinen durch das Fehlen körperlicher Aktivität ausgelöst werden, konnte bei der Transzendentalen Meditation spezifische Veränderun- 
gen - nämlich eine Atemfrequenz-Abnahme um 50\% und eine Verminderung der SauerstoffAufnahme um 40\% (Jevning et al., 1992) ermittelt werden.

Im Rahmen von Untersuchungen des ATs und anderen Entspannungstechniken fielen folgende EEG-Veränderungen auf: Israel et al. (1958) verzeichneten beispielsweise eine Abnahme von Alphawellen, die zusammen mit Betawellen den Wachzustand einer Person anzeigen, bis hin zum Verschwinden von Alphawellen über einige Minuten. Außerdem waren unregelmäßig auftretende Thetawellen ersichtlich, die physiologischer Weise im eingeschränkten Wachzustand beim Übergang zum Einschlafen oder bei Fokussierung auf ein bestimmtes Problem auftreten (Schacter, 1977). Bei Langzeit-Trainierten (sechs bis 36 Wochen) fehlten Einschlafzeichen wie K-Komplexe oder Schlafspindeln, die bei Kurzzeit-Trainierten durchaus vorkamen (Israel et al., 1958, Vaitl und Petermann, 2000). Durch regelmäßiges Training waren die Probanden in der Lage, den Übergang vom Wach- in den Schlafzustand zu unterbinden. Verminderte Schmerzempfindlichkeit und Dissoziation verschiedener Schmerzkomponenten unter Hypnose belegten Friedrich et al. (2001). Verschiedene neuere Studien zu Meditationsverfahren untermauern die Plastizität unserer Gehirnstrukturen (Davidson et al., 2003; Cahn und Polich, 2006; Hölzel et al., 2007, 2008), wobei strukturelle Veränderungen in meditationsspezifischen Regionen mithilfe bildgebender Verfahren identifiziert werden konnten.

Neben diesen hauptsächlichen Effekten sind je nach Entspannungsverfahren Veränderungen auf gastrointestinaler und humoraler Ebene - wie etwa Veränderungen des Cholesterin-, Cortison-, Prolactin- (Jevning et al., 1977, 1978) oder Blutglucosespiegels - nachgewiesen. Für die Progressive Relaxation im Speziellen konnte ein Rückgang der Cortisol-Konzentration und ein Anstieg der sIgA-Konzentration im Speichel nach einem einstündigen PR-Training ermittelt werden (Pawlow und Jones, 2005). Entspannungsverfahren wird somit auch eine gewisse Bedeutung hinsichtlich der Stärkung der Immunabwehr zugewiesen. 


\subsubsection{Anwendungsbereiche}

Entspannungsverfahren werden sowohl ambulant als auch unter stationären Bedingungen zur Prävention, Therapie und Rehabilitation zahlreicher somatischer und psychischer Störungsbilder eingesetzt. Petermann und Vaitl (2009) nennen folgende allgemeine Anwendungsfelder (s. Tab. 1). Dabei ist zu unterscheiden, ob Entspannungsverfahren als zentrale grundlegende Therapiemethode, als Baustein in einem multimodalen Programm oder als adjuvante Maßnahme in der Krankheitsbewältigung eingesetzt werden (Ohm, 2004). Als zusätzliche Behandlungsmodule können sie helfen, einen Therapieerfolg zu beschleunigen oder zu stabilisieren. In erfolgsorientierten Berufen oder generell im Alltag dienen Entspannungsverfahren dem Erhalt der Leistungs- und Arbeitsfähigkeit und leisten damit einen Beitrag zur Prävention somatischer und psychischer Erkrankungen (Petermann und Vaitl, 2009).

Tab.1: Anwendungsfelder von Entspannungsverfahren nach Petermann und Vaitl (2009)

Somatische Störungen:

- Bluthochdruck

- Koronare Herzkrankheit

- Periphere Durchblutungsstörungen (z. B. M. Raynaud)

- Asthma bronchiale

- Gastrointestinale Beschwerden

- Spannungskopfschmerzen und Migräne

- Akute und chronische Schmerzzustände

- Schlafstörungen

- Sexuelle Funktionsstörungen

- Rheumatische Erkrankungen
Psychische u. psychosomatische Störungen:

- Somatoforme Störungen

- Stressbedingte Störungsformen

- Angststörungen

- Aggressives Verhalten

- Leichte bis mittelgradige depressive Störungen

- Belastungs- und Anpassungsstörungen

- Sprechstörungen

- Aufmerksamkeitsdefizit-/ Hyperaktivitätsstörungen

- Störungen infolge Substanzmissbrauchs 


\subsection{Progressive Relaxation nach Jacobson (PR)}

\subsubsection{Die ursprüngliche Form der PR}

„There is perhaps no more general remedy than rest.”

(Jacobson, 1929)

Edmund Jacobson, der „Vater“ der Progressiven Relaxation, wurde am 22. April 1888 in Chicago geboren. Als Grundlagenforscher und praktisch tätiger Arzt sah er die Progressive Relaxation als ein Verfahren, das als Basistherapeutikum bei jeglichen Spannungszuständen auf körperlicher oder seelischer Ebene - einzusetzen sei. Dabei war er der Meinung, dass sich durch gezielte Anspannung und folgende Entspannung ein Zustand muskulärer Entspannung einstellen würde, der auf die mentale Ebene ausgedehnt werden könne. Das Entspannungsniveau als „,direkte Umkehrung von nervöser Erregung“ fungierte für Jacobson als Indikator eines entsprechenden Therapieerfolgs (Jacobson, 2006).

Sein erstes Buch aus dem Jahre 1929 - „Progressive Relaxation. A physiological and clinical investigation of muscular states and their significance in psychology and medical practice“ liefert eine detaillierte Darstellung „seiner“ Entspannungsmethode inklusive ihrer klinischen Anwendungsbereiche in Form von Fallbeispielen. Sein Ratgeber „You must relax“ (1934) fand in Amerika rasche Verbreitung. 1955 erschien mit „Immer mit der Ruhe - eine praktische Methode, die Anspannungen des modernen Lebens zu lindern“ die erste deutsche Übersetzung. Ziel seines Werkes war es, durch eine verbesserte innere Organisation und einen optimierten Energiehaushalt den Anforderungen des täglichen Lebens in entspannterer Weise gerecht zu werden - und zwar mit Hilfe dieses ,eingebauten Tranquilizers“ (Jacobson, 2006). Entspannung war für ihn ein Lebensstil und nicht nur eine Disziplin der Medizin.

Im Rahmen seiner vielfältigen Veröffentlichungen versuchte Jacobson stets, sein Konzept möglichst allen Bereichen menschlichen Lebens anzupassen, so in der Behandlung von Schlafstörungen („You can sleep well“, 1938b), als Hilfsmittel in der Mutterrolle („How to relax and have your baby“, 1959) oder im Arbeitsleben („,Tension control for businessmen“, 1963) - bis hin zur Umsetzung von Entspannung auf politischer Ebene („The peace we Americans need“, 1944). Die universelle Anwendbarkeit war Grundprinzip seines Schaffens. So sollten umfassende Kasuistiken die Wirksamkeit des Entspannungsverfahrens für affektive Störungen - wie beispielsweise Phobien oder Depression - und somatische Beschwerden wie Colitis ulcerosa, Insomnie oder Ösophagusspasmen belegen (Hamm, 2009; Derra, 2007). 
Im Mittelpunkt des PR-Konzeptes stand daher die kontinuierliche - also „progressive“ im Sinne von „fortschreitende“ - und willentliche Spannungsreduktion einzelner Muskelgruppen. Jacobson lehnte dabei explizit die Verwendung suggestiver Elemente ab (Hamm, 2009). Die Kultivierung eines Muskelsinns stand als wichtigstes Ziel im Fokus (Jacobson, 1929; Derra, 2007). Aufgabe des Übenden war dabei zuerst das Erlernen einer verbesserten Körperwahrnehmung. Durch Anspannung und Fokussierung auf das Gefühl der Verspannung sollte er diese erstmals wahrnehmen, um dann im zweiten Schritt eine gezielte Entspannung erreichen zu können. Die Sensibilisierung für muskuläre Gegebenheiten war Grundlage für das weitere Vorgehen. Das Prinzip der Spannungsminderung stellte einen weiteren Schritt auf dem Weg der Entspannung dar: Der Übende wurde mit zunehmender Erfahrung dazu angeleitet, immer feinere Kontraktionsunterschiede wahrzunehmen - zuerst mittels bestimmter Muskelbewegungen, dann durch Konzentration auf den ruhenden Muskel - bis schließlich die verbleibende Restspannung auch noch Auflösung erfahren sollte (Derra, 2007). Dabei betonte Jacobson die Unabhängigkeit von Therapeuten sowie die Ablehnung jeglicher Suggestion bei unabdingbarer Eigenständigkeit des Übenden (Jacobson, 1929).

Die Muskelkontraktion umfasste bei Jacobson jeweils ein bis zwei Minuten, wobei er besonderen Wert auf die Differenzierung zwischen Verspannung (,strain“) und Spannung (,tension“) legte. Daran anschließend folgte eine drei- bis vierminütige Phase der Entspannung, wobei Jacobson mit der Formulierung „go negative“ die gewünschte Passivität besonders betonte. Neue Empfindungen sollten sich ausbreiten können, während der Übende die passive Konzentration auf das Körperinnere richtete. Nach 25-30 Minuten und zweimaliger Wiederholung der Anspannungs-Entspannungszyklen sollte der Patient den Rest der Stunde ruhen - wobei eine Übungsstunde 50-60 Minuten dauerte.

Die Übungen wurden zuerst im Liegen, dann im Sitzen durchgeführt, wobei die Stellung der Augenlider flexibel gehandhabt werden konnte (Derra, 2007). Nach umfassender Anleitung der Trainierenden konnte selbstständig anhand eines umfangreichen Übungsplans gearbeitet werden. Das Gesamtprogramm umfasste dabei mehr als 80 Übungen und zweimal wöchentliche Einzelsitzungen zur Besprechung von Übungsproblemen anhand von Protokollen. Pro Sitzung sollte mit nur einer Muskelgruppe geübt werden - und dies täglich mindestens 60 Minuten. Der dritte Tag - die „Null-Periode“ - wurde nur der Ruhe gewidmet (Jacobson, 1934; Derra, 2007).

Jacobson führte die erste Übung mit der linken Hand durch. Die rechte Arbeitshand stellte er bewusst zurück. Von den gezielten Handübungen ging er zum gesamten Arm über; den Ar- 
men folgten die Beine. Das Vorgehen beinhaltete Übungen von außen nach innen, von großen Muskelgruppen zu kleineren, von der Peripherie nach zentral, von grober zu feiner Wahrnehmung (Derra, 2007). Mit Übergang vom Liegen in die Sitzposition - verbunden mit Übungen für Rumpf- und Nackenmuskulatur (inklusive Atemübung) - führte Jacobson das Prinzip der differentiellen Entspannung ein. Der Übende sollte dabei versuchen, während alltäglicher Arbeiten sekundäre Muskelspannungen - nämlich die Muskelverspannungen - zu lösen und nur die zur Ausführung der Tätigkeit notwendigen primären Muskelspannungen aufrecht zu erhalten. Dieses Prinzip der differentiellen Entspannung findet sich in einigen neueren Versionen der PR wieder (Hamm, 2009).

Nach Jacobson setzte mit den Übungen zu Augen- und Sprechmuskulatur die übertragende Wirkung der körperlichen und nun mehr seelischen Entspannung ein. Im Rahmen der nachfolgenden Imaginationsübungen schlossen sich an die Imagination eines emotionsfreien $\mathrm{Ob}$ jektes Imaginationen aus vertrauter Umgebung und schließlich emotionsgeladener, subjektiv berührender Inhalte an (Derra, 2007).

Die Hauptproblematik lag damals wie heute in der mangelnden Motivation zu regelmäßiger Anwendung und kontinuierlichem Training. Auch wenn Jacobson selbst durch seinen hohen Selektionseffekt (Privatpatienten und Mitarbeiter) eher mit motivierten Probanden arbeitete, blieb seine Methode aus praktischer Sicht zu umfangreich und erfuhr dementsprechend keine umfassende Durchsetzung. Wenn auch die Evaluation seiner Techniken aus heutiger wissenschaftlicher Sicht zu wünschen übrig ließ, gebührt Edmund Jacobson als Begründer der Progressiven Relaxation und Pionier auf dem Gebiet der Entspannungsforschung hohe Anerkennung (Payne, 1998; Derra, 2007).

\subsubsection{Modifizierte Versionen der PR}

Die heute angewandte Form der Progressiven Relaxation nach Jacobson basiert auf vereinfachenden und alltagsnäheren Modifikationen der Originalform. So entwickelte Wolpe in den 50er Jahren das Konzept der Gegenkonditionierung nach dem Prinzip der reziproken Hemmung zur Behandlung von Angststörungen im Rahmen der Systematischen Sensibilisierung (Wolpe, 1958; Derra, 2007). Der Patient sollte in Therapiesitzungen angehalten werden, einen Entspannungszustand aufzubauen, der als inkompatible Reaktion physiologischer Weise der Furcht entgegenstünde (stufenweise Angstreduktion nach Angsthierarchie). Wolpes Kurzform 
beinhaltete nach umfassender Einführung insgesamt sechs Sitzungen mit Übungen zu maximal 20 Muskelgruppen unter Zuhilfenahme unterstützender Formulierungen zur vertiefenden Loslösung von äußeren Reizen. Schließlich wurden verschiedene Muskelgruppen zusammengefasst, um eine alltagstauglichere Variante anbieten zu können. Aufgrund des erleichterten Alltagstransfers und der therapeutenunabhängigen selbstständigen Durchführung erfuhr diese Version regen Zuspruch (Hamm, 2009; Derra, 2007).

Die von Wolpe bereits angesprochenen Suggestionen wurden im Verlauf vor allem durch Paul (1969) besonders hervorgehoben. Signalworte während der Exspirationsphase sah er als geeignetes Mittel an, um das Prinzip der konditionierten Entspannung zu verwirklichen. Der Patient sollte durch regelmäßiges Üben fähig sein, sich in stressigen Situationen durch sein Signalwort sozusagen ,auf Kommando“ in einen Entspannungszustand zu versetzen („,cuecontrolled-relaxation“). Goldfried (1971) kombinierte schließlich drei Verfahren: Im Rahmen der sogenannten angewandten Entspannung sollte es dem Patienten gelingen, Kurzformen möglichst rasch in den Alltag zu integrieren, sein eigenes Signalwort im Sinne der konditionierten Entspannung einzusetzen und erste Anzeichen von Anspannung im Alltag zu erkennen und ganz nach dem Prinzip der differenziellen Entspannung zu lösen.

Bernstein und Borkovec kritisierten die vielen unterschiedlichen Anwendungsformen und die mangelnde wissenschaftlich belegte Wirksamkeit (Bernstein und Borkovec, 1975, 2002) und schufen ein Standardwerk, das als Grundlage des heutigen Vorgehens gilt. Als Ratgeber und Lehrbuch liefert ihr Werk „Entspannungstraining - Handbuch der progressiven Relaxation“ (in deutscher Ausgabe 1975, Original 1973) detaillierte Informationen über diese Entspannungstechnik in patientengerechter Form mit vielen Anwendungshinweisen.

Bernstein und Borkovec legten wie Wolpe Jahre zuvor den Schwerpunkt auf die Muskulatur der Extremitäten und gingen von einem Pendeleffekt aus (Hamm, 2009; Derra, 2007): Durch immer stärker werdende Anspannung sollte sich eine stetig vertiefende Entspannung einstellen. Die beiden amerikanischen Psychologen verkürzten die Anzahl der Übungen - von 16 auf sieben und schließlich vier Muskelgruppen - und die Zykluszeit auf 45-50 Sekunden. Neutrale Äußerungen des Therapeuten wurden suggestiven Formulierungen vorgezogen. Der Therapeut sollte durch gezielte Sätze dem Übenden lediglich den Entspannungsprozess verdeutlichen (Bernstein und Borkovec, 2002). Im Gegensatz zur Jacobson`schen Variante des Einzelunterrichts empfahlen sie auch die Durchführung von Gruppensitzungen. Besonderen Wert wurde auf das klärende Gespräch nach den 6-10 Sitzungen und die Wahl geeigneter Rahmenbedingungen gelegt. In der abschließenden Phase der Entspannung durch Vergegen- 
wärtigung sollte ohne Anspannungsphase nur aus der Status quo-Situation heraus entspannt werden, um aus dem vorhandenen Anspannungsniveau heraus Erleichterung zu erfahren. Zum Erlernen der differenziellen Entspannung im Alltag wurde ein Stufenplan erarbeitet (Derra, 2007).

Zu Beginn der 1930er Jahre wurde das Augenmerk auf kognitive Verfahren und Selbstkontrolltechniken gelegt. In diesem Sinne gewann die Progressive Relaxation als „,coping skill“ zunehmende Bedeutung in der Bewältigung stress- oder angstauslösender Situationen (Lazarus und Launier, 1981; Hamm, 2009). Östs (1987) „,angewandte Entspannung“ und Lichtsteins (1988) „Selbstkontroll-Entspannung“ entstanden vor diesem Hintergrund. Öst (1987) betonte in Anlehnung an die Arbeiten Goldfrieds (1971) die notwendige Alltagstauglichkeit des Entspannungsverfahrens. Eine praktikable Kurzversion sollte im Sinne eines „Selbstkontrolltrainings“ fungieren (Derra, 2007; Öst, 1987). Seine „angewandte Entspannung“ umfasste fünf Schritte - meist in Gruppensetting zu erlernen - wobei dem Entspannungstraining ein Aufklärungsgespräch über die Grundlagen und Ziele des Entspannungstrainings vorausging (Hamm, 2009; Derra, 2007).

Tab.2: Angewandte Entspannung nach Öst

1. PR-Kurzform mit 12 oder 16 Muskelgruppen im Sitzen, 2x 15min täglich

2. Lösende Entspannung: ohne vorherige Anspannung mit besonderer Beobachtung der Atmungszyklen (5-7min)

3. Stichwortgesteuerte Relaxation: durch konditionierte Entspannungsreaktion mittels Schlüsselwort (2-3min)

4. Differenzielle Entspannung: im Alltag im Sitzen, Stehen und Umhergehen (60-90sek)

5. Schnelle Entspannung: innerhalb 20-30sek, 15-20x täglich

Durch regelmäßiges Üben könne auf diese Weise ein niedrigeres alltägliches Anspannungsniveau erreicht werden. Erst nachdem diese Phasen im Rahmen von 8-12 Sitzungen sehr individuell vermittelt und erlernt wurden, schloss sich die Phase des Anwendungstrainings im Sinne der Angstreduktion in Gefahrensituationen an. Aufgrund des speziellen, an individuelle Bedürfnisse angepassten und spezifisch auf Angststörungen ausgerichteten Aufbaus konnte sich diese Version der Progressiven Relaxation jedoch nicht durchsetzen. 


\subsection{Wirksamkeit der Progressiven Relaxation}

Die Progressive Relaxation stellt das am besten untersuchte Psychotherapieverfahren dar, dessen klinische Wirksamkeit durch vielfältige Studien belegt ist (Grawe et al., 1994; Derra, 2007). Während Jacobson bei der Evaluation seines Verfahrens auf Kasuistiken zurückgriff, existieren heute zahlreiche Studien zur Wirksamkeit der modifizierten Versionen. Problematisch ist jedoch die unüberschaubare Vielfalt der Anwendungsmöglichkeiten, sodass die Vergleichbarkeit der vorhandenen Studien nur annähernd gegeben ist (Derra, 2007). Umfassende Standardisierungen zu sämtlichen Komponenten fehlen bis heute. Widersprüchliche Befunde - gerade zu physiologischen Effekten der PR - sind fast zwangsläufige Konsequenz (Hamm, 2009). Die Vielfalt von Einzeluntersuchungen mit teilweise gegensätzlichen Ergebnissen steht einer fundierten Evaluation entgegen (Hamm, 2009). Diese Inkonsistenz lässt sich zum Teil durch die unterschiedlichen Induktionsprozesse erklären. Instruktionen vom Tonband haben geringere physiologische Effekte als Instruktionen direkt vom Therapeuten selbst (Paul und Trimble, 1970), im Einzelsetting erhält der Wirkfaktor „Arzt-Patient-Beziehung“ gegenüber Gruppenprogrammen stärkere Bedeutung (Grawe, 1994).

Das Ziel von Leitlinienkonferenzen war daher der Abschluss eines Minimalkonsenses zur PRAnwendung. Dieser liefert bisher Vorgaben zur Schaffung strukturierter Rahmenbedingungen im klinischen Alltag mit besonderer Betonung aufklärender Vorinformationen und regelmäßiger Effektkontrolle durch Nachbefragung sowie Problemklärung beim eigenständigen Üben (Ohm, 2004). Grundlagenstudien, die die physiologischen Effekte der PR in verschiedenen Indikationsbereichen ermitteln, stehen klinischen Studien zur Wirksamkeit der PR in der Therapie verschiedener Störungsbilder gegenüber, auf die im Folgenden näher eingegangen werden soll.

\section{Klinische Effektivität}

Hyman et al. (1989) zeigten bereits im Jahre 1989 in ihrer Meta-Analyse zur klinischen Wirksamkeit von Entspannungsverfahren, dass die Progressive Relaxation einen Großteil der angewandten Entspannungstechniken ausmacht. In 48 eingeschlossenen Studien wurde die PR in 38,2\% der Fälle angewandt. Die Hauptanwendungsbereiche der Metaanalyse von Carlson und Hoyle (1993) waren Kopfschmerzen, Bluthochdruck und Nebenwirkungen von Chemotherapie. Die durchschnittliche Effektstärke betrug r=0.40. Individuelle Vermittlung, häusliche Unterstützung mittels Tonbändern sowie Trainingsdauer und Anzahl der Trainings- 
stunden wurden als Moderatorvariablen ermittelt, die zu einer Verbesserung der PR-Effekte führten.

Die Anwendungsbereiche der Progressiven Relaxation lassen sich zu drei Teilbereichen zusammenfassen: chronische Schmerzzustände, somatische Erkrankungen und psychische Störungen.

Chronische Schmerzzustände. Die Progressive Relaxation findet in der Therapie akuter und chronischer Schmerzzustände häufige Anwendung. Die klinische Effektivität variiert dabei erheblich je nach Schmerzform. Kwekkeboom und Gretarsdottir (2006) fassten in ihrem systematischen Review Studien zur Entspannungstherapie von chronischen Schmerzzuständen bei Erwachsenen ab dem Jahre 1996 zusammen. In acht von 15 Studien zeigten sich signifikant-positive Effekte von Entspannung, wobei sich die PR in den Bereichen Arthritis, chronische Rückenschmerzen und Beinschmerzen in der Schwangerschaft als effektiv erwies (Kwekkeboom und Gretartsdottir, 2006). Weitere Studien zur Wirksamkeit Progressiver Relaxation bei verschiedenen Schmerzformen fasst die Tabelle 3 zusammen. Die schmerzreizdämpfende Wirkung der PR konnte jüngst durch die Arbeit von Emery et al. (2008) objektiviert werden. Der nozizeptive Flexionsreflex (NFR) war Gegenstand der Untersuchung. Die jungen, gesunden Probanden zeigten nach einem PR-Training einen signifikanten Anstieg der NFR-Schwelle im Vergleich zur Kontrollgruppe. Außerdem gaben sie ein vermindertes Stressempfinden nach dem Kurs an (Emery et al., 2008).

Somatische Erkrankungen. Im Rahmen körperlicher Symptome finden Entspannungsverfahren vielfältige Anwendung. Die Tabelle 4 liefert eine Übersicht einiger Studien zur Wirksamkeit von Progressiver Relaxation auf somatischer Ebene.

Schwangerschaft. Beispielhaft soll hier die Studie von Janke aus dem Jahre 1999 genannt werden, die positive Effekte hinsichtlich des Risikos einer Frühgeburt belegen konnte: Frauen, die Progressive Relaxation zuhause praktizierten, hatten ein besseres Outcome bezüglich Geburtsgewicht des Neugeborenen, Gestationsalter bei Geburt und Anzahl verlängerter Schwangerschaften. 
Tab.3: Studien zur Wirksamkeit bei verschiedenen Schmerzformen und deren Ergebnisse Schmerzform:

Spannungskopfschmerz:

- Verbesserung von Schmerzverhalten und Schmerzempfinden (Philips und Hunter, 1981; Murphy et al., 1990)

- Abnahme der maximalen Kopfschmerzintensität, Zunahme schmerzfreier Tage (Blanchard et al., 1982)

- Reduktion von Analgetika-Gebrauch (Blanchard et al., 1990)

Migräne:

- Verminderung der Dauer und Häufigkeit von Migräneattacken (Mitchell und Mitchell, 1971; Turner und Chapman, 1982a, 1982b; Janssen und Neutgens, 1986)

Rückenschmerzen:

- Linderung von Beschwerden, Verbesserungen hinsichtlich Depression, Aktivität und Medikamenteneinnahme (Linton und Götestam, 1984)

- Positive Effekte auf Gesundheitsstatus und subjektives Schmerzempfinden (Turner, 1982)

Rheumatische Beschwerden:

- Signifikante Schmerzreduktion und Verminderung der Mobilitätseinschränkungen bei Osteoarthritis (Baird und Sands, 2004) 
Tab.4: Studien zur Wirksamkeit bei somatischen Erkrankungen und deren Ergebnisse

\section{Somatische Erkrankungen:}

Essentielle Hypertonie:

- Antihypertensive Wirkung (17,8/9,7mmHg bei Brauer et al., 1979; Southam et al., 1982; Hoelscher, 1987; Jacobson, 1947)

- Herzfrequenz-Reduktion (2,35Schläge/min), RR-Reduktion $(5,44 / 3,48 \mathrm{mmHg})$, Verringerung des Stressempfindens und verbesserte Wahrnehmung der eigenen Gesundheit (Sheu et al., 2003)

Kardiovaskuläre Erkrankungen:

- Positive Effekte auf Herzfrequenz, Ängstlichkeit, Stressreduktion und subjektive Zufriedenheit (Wilk und Turkoski, 2001)

- Aufrechterhaltung der Hauttemperatur angesichts eines Kälteereignis bei M. Raynaud (Keefe et al., 1980)

- Verminderte Ruheherzfrequenz, erhöhte Herzfrequenzbreite, Anstieg des HDL-Cholesterins, bessere Belastbarkeit nach einem ischämischen Ereignis, verringerte Häufigkeit von Anginapectoris-Anfällen, Arrhythmie-Phasen und belastungsinduzierten Ischämien, schnellere Rückkehr in die Arbeitswelt, weniger kardiale Ereignisse und Todesfälle (Van Dixhoorn und White, 2005)

- Positive Auswirkungen auf Distress und Lebensqualität bei chronischer Herzinsuffizienz (Yu et al., 2007a, 2007b, 2010)

HIV:

- Positive Effekte auf T-Zell-Aktivität, Selbstachtung, Stimmung, Angst (Taylor, 1995)

- Verbesserungen bezüglich Symptomen, Lebensqualität und Immunsystem (Eller, 1999)

Bronchopulmonale Erkrankungen:

- Anstieg exspiratorischer Peak-Flow- und FEV1-Wertebei Asthmatikern (Huntley et al., 2002)

- Rückgang von Asthma-Symptomen, Angst, Depression und Verbesserung der Lebensqualität (Grover et al., 2002)

- Positive Effekte hinsichtlich Blutdruck, Herzfrequenzbreite und Lungenparametern bei AsthmaPatientinnen (Nickel et al., 2005) und schwangeren Asthmatikerinnen (Nickel et al., 2006)

- Reduktion von Angst und Dyspnoe, Verbesserungen hinsichtlich Blutdruck, Herz- und Atemfrequenz bei exazerbierter COPD (Singh et al., 2009) 
Haut- und Gastrointestinale Erkrankungen:

- Positive Auswirkungen auf dermatologische Erkrankungen wie Psoriasis und Akne (Fried und Hussein, 2008)

- Abnahme subjektiver Stresssymptome und verbesserte Ulkus-Heilung im Rahmen eines integrativen Stress-Management-Programms bei Patienten mit Magenulcera (Han, 2002)

- Niedrigere Blutglucosespiegel und verminderte Stresslevel bei insulinpflichtigen Diabetikern (Lammers et al., 1984)

Schlafstörungen:

- Verbesserungen hinsichtlich Zeit bis zum Einschlafen, Anzahl der Aufwachphasen und Gefühl des Ausgeruhtseins am Morgen (Borkovec und Fowles, 1973)

- Verbesserte Schlafqualität und weniger Wachzeiten während der Nacht bei Studenten mit Schlafstörungen (Means et al., 2000)

Maligne Erkrankungen:

- Schmerzreduktion bei onkologischen Patienten unter Radio-/Chemotherapie und Opioid-Einnahme (Kwekkeboom et al., 2008)

- Anxiolytische Wirkung und signifikante Verbesserungen depressiver Symptome, wirksame Alternative zur Therapie mit Benzodiazepinen (Holland et al., 1991)

- Rückgang von Chemotherapie-assoziierter Übelkeit und Erbrechen (Molassiotis, 2000; Molassiotis et al., 2002; Yoo et al., 2005)

- Positive Auswirkungen auf Schlafqualität und Fatigue-Symptome bei Brustkrebspatientinnen (Demiralp et al., 2010)

Psychische Erkrankungen. Es liegen zahlreiche Studien zur Wirksamkeit von Progressiver Relaxation in der Therapie psychischer Erkrankungen - der Hauptdomäne dieser Entspannungstechnik - vor. In der Behandlung depressiver Erkrankungen und akuter Belastungsreaktionen kommt diesem Entspannungsverfahren vor allem als adjuvantes und unterstützendes Behandlungsmodul zur Ergänzung psychologischer Basisversorgung besondere Bedeutung zu (Jorm et al., 2008; Freyth et al., 2010). Im Folgenden soll schließlich detaillierter auf den Einsatz von PR in der Therapie von Angst- und Essstörungen eingegangen werden. 


\section{Angststörungen}

Als effektives Mittel, Selbstvertrauen und Selbstkontrolle aufzubauen und Angststörungen zu lindern, konnte PR von Weber (1996) bei hospitalisierten Patienten der Psychiatrie identifiziert werden. McGlynn et al. (1999) dokumentierten im Rahmen ihrer Untersuchung von Patienten mit Schlangenphobie die positiven PR-Wirkungen während der Exposition in vivo: Im Rahmen der Metaanalyse von Manzoni et al. (2008) konnte zusammenfassend die konsistente und signifikante Wirksamkeit dieses Entspannungsverfahrens in der Verminderung von Angstgefühlen belegt werden. Verminderte Ängstlichkeit nach einem PR-Kurs bei Patienten mit akuter Schizophrenie wurde von Chen et al. (2009) publiziert. Darüber hinaus wurde ein Anstieg der durchschnittlichen Körpertemperatur nach dem Entspannungstraining ermittelt. Laut Jorm et al. (2004) liegen die besten Wirksamkeitsevidenzen für PR bezüglich Generalisierter Angststörung, Panikstörung, Zahnphobie und Prüfungsangst vor.

\section{Essstörungen}

Ein erster Schritt in der Behandlung von Anorexia nervosa ist regelmäßige Ernährung zum Erreichen eines festgelegten Mindestgewichts. Solche Interventionen rufen bei Patienten große Angstgefühle hervor. Shapiro et al. (2008) untersuchten daher die Auswirkungen von Entspannungstechniken auf postprandiale Angstzustände von 64 Anorexie-Patientinnen. 15 Tage lang erhielten sie nach dem Mittagsessen einen Entspannungskurs. Sowohl die Kontrollgruppe, die irgendeiner Beschäftigung wie Lesen o. ä. nachging, als auch die Interventionsgruppen erlebten Verbesserungen hinsichtlich Angst, Entspannung, Völlegefühlen und Grübeln über das eigene Gewicht. Die aktiven Entspannungstechniken - nämlich PR, Gelenkte Imagination (GI) und selbstgeführte Entspannung - konnten jedoch zu einer signifikanten Reduktion von Angst und einer signifikanten Zunahme von Entspannung gegenüber der Kontrollgruppe führen.

Zusammenfassend lässt sich feststellen, dass Progressive Relaxation nicht nur in der Behandlung der eigentlichen Erkrankung, sondern auch in der Therapie bestimmter Krankheitskomponenten oder sekundärer Symptome hilfreich ist - wie gerade das letzte Beispiel noch einmal verdeutlicht. In der Therapie der Krankheit selbst, aber auch im Verlauf der Krankheitsbewältigung kann Progressive Relaxation als effektive, kostengünstige und nebenwirkungsarme Alternative oder adjuvante Intervention wirkungsvoll eingesetzt werden. 


\subsection{Anwendungsbereiche der Progressiven Relaxation}

Bereits Edmund Jacobson war sich des breiten Anwendungsspektrums der Progressiven Relaxation bewusst. So plädierte er für den Einsatz dieses Entspannungsverfahrens bei allen spannungsbedingten Erkrankungen - ganz gleich ob somatisch oder psychisch bedingt (Jacobson, 2006). Derra (2007) bezeichnete es gar als „Basispsychotherapeutikum“, das zu einer konditionierten Entspannungsreaktion, differenzierten Körperwahrnehmung, mentalen Frische, verbessertem Stressabbau, Distanzierung von inneren und äußeren Störvariablen, trainierter Aufmerksamkeitslenkung und erlebter Selbstkontrolle führt. Allerdings können diese Ressourcen nur durch regelmäßiges Üben und kontinuierliche Anwendung ausgeschöpft werden. Integration in den Alltag, am besten gezielt durch persönliche Triggerfaktoren, und tägliche Übungsroutine sind Voraussetzungen für eine prophylaktische und erfolgreiche Anwendung.

Gerade im Rahmen einer präventiven Anwendung favorisiert Derra (2007) den möglichst frühen Beginn des PR-Trainings: So können bereits Kinder ab dem vierten Lebensjahr in Kinderkursen angeleitet werden. Progressive Relaxation eignet sich besonders für den präventiven Einsatz in leistungsorientierten Bereichen wie Beruf, Schule, Studium, Sport und Prüfungsvorbereitung, aber auch im Rahmen anderer spannungsgeladener Situationen wie Schwangerschaft, Geburt oder operativen Eingriffen. Die jeweilige Erwartungshaltung sollte vorher genau eruiert werden. Erfolgsdruck durch zu hohe Erwartungshaltung ist ebenso wenig förderlich wie Motivationsmangel (Derra, 2007). Krampen (2002b) konnte belegen, dass bei Patienten mit intrinsischen Teilnahmemotiven Kursabbrüche seltener sind als bei Patienten mit extrinsischen Motiven und dass der Lern- und Transfererfolg auch von den bestehenden Vorerfahrungen abhängig ist (vgl. Kap. 5.2).

Ohm (2004) fasst die klinischen Anwendungsgebiete zusammen, die von der Fachgruppe „Entspannungsverfahren“ des Berufsverbandes deutscher Psychologinnen und Psychologen (BDP) im Rahmen der Leitlinienkonferenzen im Jahre 2001 erstellt wurden (s. Tab. 5). Dem präventiven Einsatz von PR in der allgemeinen Gesundheitsvorsorge zur Bereitstellung von Ressourcen kommt dabei besondere Bedeutung zu (Ohm, 2004). Favorisiert werden Gruppen bis maximal 12 Personen und 8-12 Sitzungen mit jeweils 25-100 Minuten.

Es ist zu unterscheiden, ob die Progressive Relaxation zu einem direkten Beschwerdeabbau etwa wie bei Spannungskopfschmerz - oder aber zur Unterstützung in der Krankheitsbewältigung wie beispielsweise im Falle einer HIV-Infektion eingesetzt wird. 
Tab.5: Anwendungsgebiete der Progressiven Relaxation (Ohm, 2004)

- Aggression

- Alkoholismus

- Alzheimer Erkrankung

- Angststörungen (adjuvant bei Phobien)

- Asthma

- Bruxismus

- Chronische Müdigkeit

- Colon irritable

- Depression

- Diabetes mellitus Typ II

- Essstörungen

- Gedächtnisstörungen

- Glaukom

- Hauterkrankungen

- HIV
- Hypertonie

- Koronare Herzkrankheit

- Krebs

- Migräne

- Multiple Sklerose

- Parkinson-Erkrankung

- Phantomschmerz

- Posttraumatische Belastungsstörung

- Schlafstörungen

- Schmerzen, (Rücken-, rheumatische, Spannungskopfschmerzen, Migräne)

- Schwindel

- Sprachstörungen

- Störungen des Immunsystems

- Tinnitus

- Zwangsstörungen

Desweiteren werden unspezifische Beschwerden wie Beklemmungen oder Verspannungen durch Distress genannt. Ergänzende Indikationsbereiche finden sich bei Olschewski (1996):

- Allgemeine Gesundheitsprophylaxe

- Nervosität

- Prüfungsangst und frei flottierende Ängste

- Stressulkus

- Absetzen oder Reduzieren von Schmerzmitteln oder Tranquilizern

Krampen (2004) hält Progressive Relaxation bei folgenden Störungsbildern aus dem F-Kapitel nach ICD-10 indiziert (s. Tab.6). Er differenziert hierbei in Vor- (V), Begleit- (B) und Nachbehandlung (N) und setzt somit das Entspannungsverfahren als adjuvante Therapie in den Kontext anderer (Basis-) Therapien. 
Tab.6: Anwendungsbereiche von PR bei psychischen Störungen nach Krampen (2004)

- Somatisierungsstörung $(V / B / N)$

- Direkt durch Muskelverspannungen bedingte Störungen $(\mathrm{V} / \mathrm{B} / \mathrm{N})$

- Phobische Störungen (V/B/N)

- Belastungsreaktionen und Anpassungsstörungen $(B / N)$

- Neurasthenie (V/B/N)

- Sexuelle Funktionsstörungen (V/B/N)

- Substanzabusus ohne Abhängigkeit $(\mathrm{B} / \mathrm{N})$
- Störung der Impulskontrolle (V/B/N)

- Intelligenzminderungen (V/B/N)

- Entwicklungsstörungen hinsichtlich schulischer Fähigkeiten (B/N) und motorischer Funktionen (V/B/N)

- Enuresis (V/B/N)

- Enkopresis (V/B/N)

- Stereotype Bewegungsstörungen $(\mathrm{V} / \mathrm{B} / \mathrm{N})$

- Poltern (V/B/N)

Krampen (2004) favorisiert Progressive Relaxation als wenig autosuggestives, aktives Verfahren, das primär die körperliche Ebene der Entspannungsreaktion betont, wenn muskulär bedingte Beschwerden vorhanden sind, ein Wunsch nach körperlicher Aktivität und differenzierter Schulung des Muskelsinns und ein hoher kurzfristiger Interventionsbedarf besteht. Dabei plädiert Krampen für ein mehrstufiges differenzialdiagnostisches Vorgehen zur Optimierung der differentiellen Indikation von PR und AT. Nach dem ersten Schritt der Erfassung einer allgemeinen Indikationsstellung schließt sich im zweiten Schritt die Erfassung der Vorerfahrungen mit Entspannungsverfahren an. Progressive Relaxation sollte dabei vor allem bei positiven Vorerfahrungen mit PR oder anderen körperorientierten Verfahren oder negativen Vorerfahrungen mit AT oder anderen meditativ/imaginativen Verfahren eingesetzt werden. Die Teilnahmemotive können im dritten Schritt erfragt werden. Der vierte und letzte Schritt beinhaltet die Erfassung änderungssensitiver Symptome, wobei Progressive Relaxation als Methode der ersten Wahl bei erhöhter Nervosität und innerer Anspannung identifiziert werden konnte. Als gute Screening-Fragebögen können dabei die auch in der vorliegenden Studie eingesetzten Fragebögen ET-ANAM (Krampen, 2002a) und ET-SYM (2003) - modifiziert nach AT-EVA (Krampen, 1991) - Anwendung finden.

Kontraindikationen bestehen in den seltensten Fällen. Anwendungsprobleme wie innerliche Blockaden haben meist lediglich zur Folge, dass Entspannungsübungen nicht wirksam werden und sich eine gewisse Enttäuschung beim Übenden einstellt (Derra, 2007). Es kann sinnvoll sein, Hilfestellungen anzubieten, das Gruppen- durch ein Einzelsetting zu ersetzen, das Ent- 
spannungsverfahren $\mathrm{zu}$ wechseln oder $\mathrm{zu}$ einem späteren Zeitpunkt erneut $\mathrm{zu}$ beginnen. Ernsthafte oder gar schädliche Folgen durch Progressive Relaxation sind sehr selten. Absolute Kontraindikationen sind schwere neurologische Störungen und Krankheitsbilder, die mit einem anhaltend gestörten Realitätskontakt einhergehen (Derra, 2007).

Ohm (2004) nennt folgende absolute und relative Kontraindikationen, wobei sich relative Kontraindikationen auf akute Störungen beziehen, die zeitweilig gegen die Durchführung von Entspannungsübungen sprechen (s. Tab.7). Krampen führt desweiteren hypochondrische Störungen, autonome Funktionsstörungen, parasympathisch geprägte Störungen, wahnhafte und affektive Störungen, psychische Störungen im Wochenbett, Zwangs-, Ess- und Persönlichkeitsstörungen als relative Kontraindikationen und dissoziative Störungen und das Depersonalisationssyndrom als absolute Kontraindikationen an (Krampen, 2004).

Tab.7: Absolute und relative Kontraindikationen der PR nach Ohm (2004)

Absolute Kontraindikationen:

- Schwere Intelligenzeinschränkungen (Oligophrenie, Demenz)

- Chronische neurologische Störungen, Myotonien, Muskelspastiken, generalisierte Torsionsdystonien

- Chronifizierte Psychosen wie anhaltende wahnhafte Störungen oder Schizophrenien
Relative Kontraindikationen:

- Verwirrtheitszustände

- Neigung zu Überanstrengung

- Muskelentzündungen oder Muskelerkrankungen, die sich durch Muskelkontraktion verschlechtern könnten

- Neurologische Systemerkrankungen wie Kleinhirn- oder extrapyramidale Störungen, die sich bei Muskelkontraktionen verschlechtern könnten

- Akute Psychosen wie Manie oder Schizophrenie

Grundsätzlich verfügt die Progressive Relaxation als einfaches und alltagstaugliches Entspannungsverfahren über ein breites Anwendungsspektrum und bietet aufgrund seiner Ähnlichkeit zum Krafttraining auch gerade jungen Menschen einen guten Einstieg. 


\subsection{Psychosomatische Rehabilitation in Deutschland}

Prävention, Therapie, Pflege und Rehabilitation stellen die wesentlichen Säulen eines funktionierenden Gesundheitssystems dar. Das Ziel der Rehabilitation (Tertiärprävention) ist es, Behinderungen, bleibende Funktionsverluste oder eine Verschlimmerung der bestehenden Beschwerden zu verhüten, kompensierende Ersatzstrategien zu entwickeln, die Leistungsfähigkeit soweit möglich wieder herzustellen und eine adaptive Krankheitsverarbeitung zu ermöglichen (Walter et al., 2008; Schwartz et al., 2003; Schmid-Ott et al., 2008). Neben diesen allgemeinen Rehabilitationszielen sind folgende Ziele auf psychosomatischer Ebene zu nennen:

- Förderung des angemessenen Umgangs mit der psychosomatischen Erkrankung und deren Folgen

- Rückkehr ins Berufsleben bzw. Wiederherstellung/ Erhalt von Erwerbsfähigkeit

- Erlangen von sozialkommunikativer Kompetenz und Autonomie

- Vermeidung sozialer Abhängigkeit und zukünftiger Pflegebedürftigkeit

Die Grundlagen, Aufgaben und Ziele der Rehabilitation sind darüber hinaus im „Rahmenkonzept zur medizinischen Rehabilitation in der gesetzlichen Rentenversicherung“ (Erstversion 1992, aktualisiert 1996 und 2007) dargelegt (VDR, 2007). Das flexible System der Rehabilitation setzt sich aus ambulanten, teilstationären und stationären Angebotsformen zusammen. Mit 16.000 Betten in den Rehabilitationskliniken gegenüber 2500 Betten in der Akutversorgung findet die psychosomatische Behandlung überwiegend in rehabilitativen Einrichtungen statt (Schmid-Ott et al., 2008). In der psychosomatischen Rehabilitation liegen die stationären Aufenthaltszeiträume zwischen vier und acht Wochen, bei Abhängigkeitserkrankungen auch deutlich länger.

Diagnostik und Therapie verfolgen einen ganzheitlichen und interdisziplinären Rehabilitationsansatz, der die aktive Mitarbeit des Rehabilitanden fördert (Schmid-Ott et al., 2008). Dabei gilt der Bezugstherapeut als zentraler Ansprechpartner und steuert in Kooperation mit dem Patienten den therapeutischen Prozess (Wiegand-Grefe und Mans, 2008).

Die Gruppenarbeit - in Form von Stations-Basisgruppen, störungsspezifischen Gruppen, psychoedukativen Gruppen und eben auch Entspannungsgruppen - stellt ein weiteres wesentliches Kennzeichen psychosomatischer Rehabilitation dar.

Allgemeine und spezielle Indikationen stationärer psychosomatischer Rehabilitation werden im Folgenden tabellarisch aufgeführt (Paar und Grohmann, 2008; s. Tab.8). 
Tab.8: Allgemeine und spezielle Indikationen stationärer psychosomatischer Rehabilitation

Allgemeine Indikationen:

- Drohende Berufs- oder

Erwerbsunfähigkeit

- Therapeutisch notwendige Distanzierung zum persönlichen Umfeld

- Problemverhalten mit Verlust der Selbstkontrolle

- Vermeidungsverhalten

- Psychosomatische Erkrankungen mit somatischer Komorbidität

- Funktionsstörungen, die einer ambulanten Therapie entgegenstehen
Spezielle Indikationen:

- Depressive Störungen

- Anpassungsstörungen und Belastungsreaktionen

- Angststörungen

- Somatoforme Störungen

- Körperliche Störungen mit starker psychischer Komponente

- Teilgruppen anderer psychischer oder psychosomatischer Störungen (Persönlichkeitsstörungen, Essstörungen)

Der Phase einer intensiven medizinischen Rehabilitation kann sich eine Zeit spezifischer Nachsorgeprogramme, beispielsweise zur beruflichen Wiedereingliederung, anschließen.

Die abschließende Evaluation durchgeführter Rehabilitationsmaßnahmen ist im Sozialgesetzbuch IX vorgeschrieben (Schmid-Ott et al., 2008).

\subsubsection{Entspannungsverfahren als Bestandteil psychosomatischer Rehabilitation}

Ein wesentliches Element stationärer psychosomatischer Rehabilitation ist die Gruppenarbeit. Entspannungsverfahren stellen dabei einen effektiven und wichtigen Bestandteil der verschiedenen, störungsspezifischen und störungsunspezifischen Gruppentherapien dar. Ihnen kommt ein großer Nutzen hinsichtlich Ressourcenaktivierung und Selbstwirksamkeitserleben zu (Klinkenberg, 2008).

Entspannungsverfahren gehören als Evidenzbasierte Therapiemodule (ETM) zu den RehaTherapiestandards der Deutschen Rentenversicherung bezüglich folgender Krankheitsbilder:

- $\quad$ Koronare Herzkrankheit (I20-I25, Z95.1, Z95.5)

- Diabetes mellitus Typ II (E11) 
- $\quad$ Brustkrebs (C50)

- $\quad$ Hüft- und Knie-TEP (M16, M17, Z96.6, Z96.8, Z98.8)

- Chronischer Rückenschmerz (M42.1, M42.9, M43.0-5, M43.8-9, M47.2, M47.8-9, M48.8-9, M51.0-4, M53.8-9, M54.1, M54.3-9, M96.1, Z96.7)

In der Pilotphase:

- Alkoholabhängigkeit (ab Dezember 2009) (F10.2)

- Schlaganfall (ab 2010) (G46, I60-64)

- Depressive Störungen (ab 2010) (F32-34.1)

- Neurodermitis bei Kindern und Jugendlichen (ab Juni 2010) (L20, L28, L85.3)

Die therapeutischen Inhalte bzw. Zielsetzungen umfassen jeweils das Vermitteln und Einüben einer Entspannungstechnik (namentlich Autogenes Training oder Progressive Relaxation in Einzel- oder Gruppensetting sowie Biofeedback oder sonstige Entspannungsverfahren), das Erarbeiten individueller Nutzungsmöglichkeiten und den Transfer in den Alltag. Hinsichtlich formaler Ausgestaltung sind folgende Vorgaben zu finden:

- Mindestdauer pro Rehabilitation: mind. 180 min (bei Kindern 80 min, bei Alkoholabhängigkeit und depressiven Störungen 240 min)

- Mindesthäufigkeit pro Rehabilitation: mind. 4-mal. (bei Alkoholabhängigkeit und depressiven Störungen mind. 6-mal)

Der Mindestanteil entsprechend zu behandelnder Rehabilitanden beträgt je nach Krankheitsbild mind. 10-75\% (vgl. Reha-Therapiestandards, www.deutsche-rentenversicherung-bund .de). 


\subsubsection{Progressive Relaxation in der Fachklinik für Psychosomatische Rehabili- tation und Psychotherapie der MediClin Bliestal Kliniken, Blieskastel}

Der Kurs der Progressiven Relaxation nach Jacobson, welcher langjähriges Therapiemodul des multimodalen Behandlungsprogramms der MediClin Bliestal Kliniken und Grundlage der vorliegenden Arbeit ist, erfuhr im Laufe der Jahre einige Modifikationen. Gegenstand der Studie von Arndt aus dem Jahre 2005/2006 war die Evaluation des ursprünglichen Entspannungskurses. Dieser umfasste 6 Sitzungen zweimal wöchentlich in Gruppen von 8 - 12 Teilnehmern. Es wurde weder standardisiertes Informationsmaterial ausgegeben noch erfolgte die Durchführung nach einem systematischen Konzept mit regelmäßiger Nachbesprechung der einzelnen Übungen.

Im Jahre 2008 wurde das Entspannungstraining modifiziert. Es konnte ein umfassendes Konzept zum Aufbau des Kurses mit Ausarbeitung eines Manuals für Therapeuten entwickelt werden (Maierhofer et al., 2008). Die Abbildung 1 zeigt eine Übersicht dieses Kursaufbaus. Vor Beginn des eigentlichen Entspannungskurses absolvieren die Teilnehmer ein Grundlagenseminar zum Thema „Entspannung“. Darin erhalten sie Informationen über die Merkmale von An- und Entspannung, zu Entspannungsverfahren im Allgemeinen und Progressiver Relaxation im Speziellen, den Ablauf, die Rahmenbedingungen und die Wirkungen und Nebenwirkungen des Entspannungsprozesses. Im Anschluss erhalten die Patienten ein ausführliches Skript mit allen wichtigen Informationen des Grundlagenseminars.

Der Entspannungskurs an sich beinhaltet sechs Sitzungen zu je 45 Minuten. Ungefähr 30 Minuten sind für die jeweiligen Übungen, die restliche Zeit zur Vor- und Nachbesprechung vorgesehen. Dabei sollen etwaige Fragen und individuelle Probleme in der Gruppe geklärt sowie die Patienten zum eigenständigen Üben auch außerhalb der Kursstunden kontinuierlich motiviert werden. Die sechs Sitzungen umfassen aufeinander aufbauende Übungen. Den eigentlichen Muskelübungen geht jeweils eine Einleitungsphase voraus, in der sich durch beruhigende Instruktionen des Entspannungstherapeuten ein Gefühl des Zur-Ruhe-Kommens einstellen soll. Den eigentlichen Übungen - wobei die Aufmerksamkeit besonders auf den Wechsel von anstrengender Anspannung und erlösender Entspannung gerichtet werden soll schließt sich eine Phase des „Zurückholens“ aus dem Entspannungszustand an. Dies geschieht in vier Schritten, wobei zuerst die Hände, dann Hände und Arme und schließlich der ganze Körper bewegt werden soll, bevor im letzten Schritt mit Öffnen der Augen der Abschluss der Entspannungsübung erfolgt. 


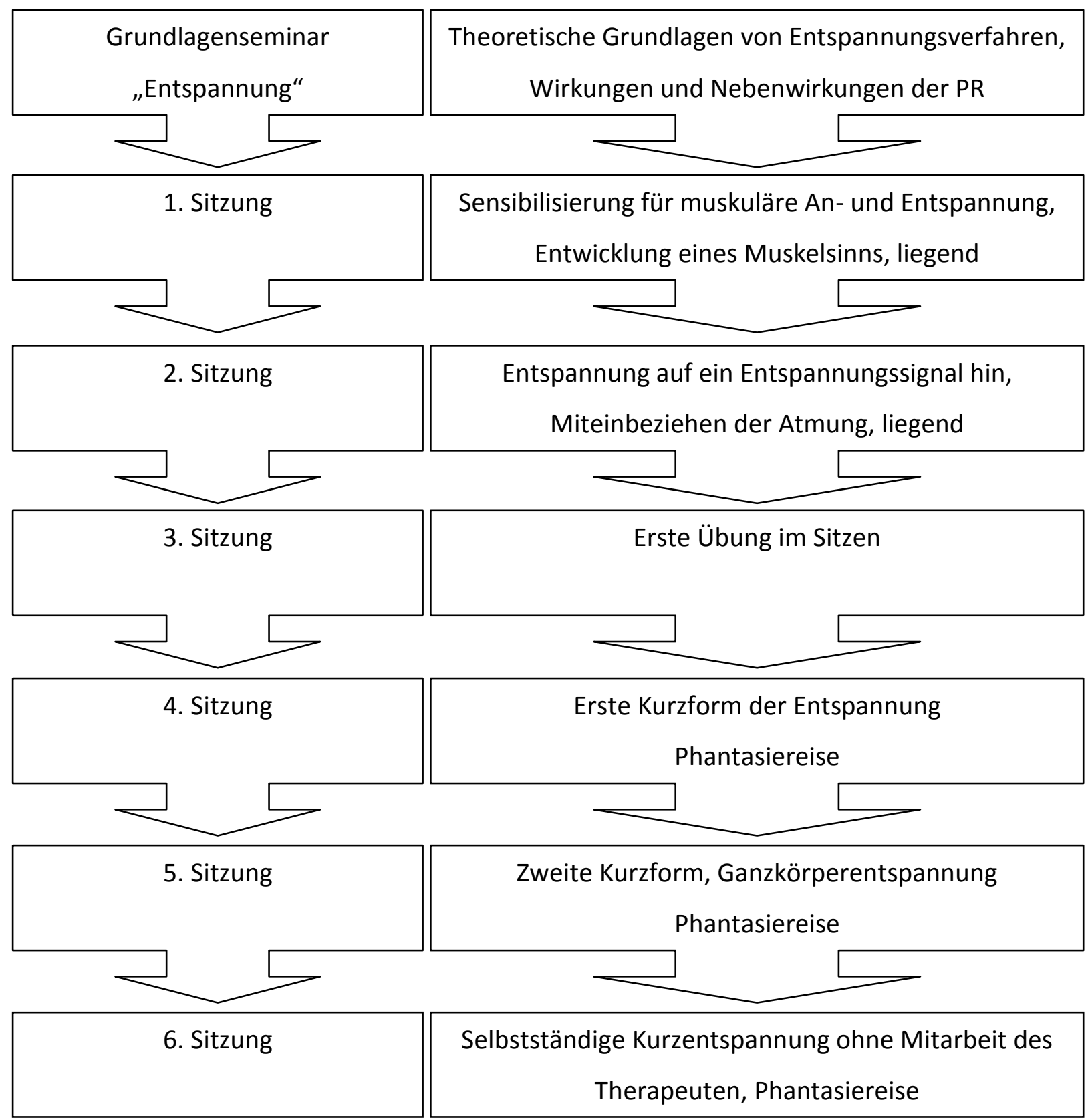

Abb.1: Aufbau des Entspannungskurses der Psychosomatischen Fachklinik der MediClin Bliestal Kliniken in Blieskastel

Die erste Stunde dient der Klärung möglicher organisatorischer Fragen sowie der Einführung der Patienten. Es wird im Liegen mit einigen wenigen Muskelgruppen - intensiv mit beiden Händen - gearbeitet, um erstmals ein deutliches Gefühl für An- und Entspannung empfinden zu lernen. Eine erste Schulung des Muskelsinns soll ermöglicht werden.

In der zweiten Sitzung wird abermals im Liegen mit allen wesentlichen Muskelgruppen gearbeitet - diesmal unter Zuhilfenahme eines individuellen Entspannungssignals. Darüber hinaus wird die Atmung gezielt an den Zyklus aus An- und Entspannung gekoppelt (einatmen und gleichzeitig anspannen - ruhige Atmung während der Anspannungsphase - Entspan- 
nungssignal geben - entspannen und gleichzeitig ausatmen). Im Sinne der stichwortgesteuerten Entspannung nach Öst kann sich durch Verwendung eines Schlüsselwortes eine konditionierte Entspannungsreaktion aufbauen.

In der dritten Sitzung finden die Entspannungsübungen erstmals im Sitzen statt, um eine bessere Alltagstauglichkeit und damit auch einen verbesserten Alltagstransfer zu ermöglichen. Die Patienten werden dabei kontinuierlich auf die Notwendigkeit des regelmäßigen Übens hingewiesen.

Eine erste Kurzform über zweimal fünfzehn Minuten mit Zusammenfassung verschiedener Muskelgruppen wird in der vierten Stunde vermittelt. Nachdem der erste Durchgang von leitenden Instruktionen des Therapeuten begleitet war, sollen im zweiten Durchgang nur noch die zu entspannenden Muskelgruppen vorgegeben werden. Die Patienten versuchen, die Übungen an sich selbstständig durchzuführen. Diese Sitzung wird schließlich mit einer Phantasiereise (Spaziergang über eine Wiese) beendet.

In der fünften Sitzung wird die Kurzform der Ganzkörperentspannung vermittelt. Alle Muskelgruppen sollen gleichzeitig an- und schließlich entspannt werden. Die Patienten werden dazu angeleitet, sich dabei in eine reale Situation zu versetzen, um den Alltagstransfer zu erleichtern. Auch diese Stunde besteht aus zwei Durchgängen. Der zweite Durchgang wiederholt die Kurzform der letzten Stunde, ist erneut durch selbstständige Durchführung bei starker Zurückhaltung des Therapeuten gekennzeichnet und geht nahtlos in eine Phantasiereise über (Spaziergang am Strand).

Die sechste und letzte Sitzung des Entspannungskurses wiederholt nochmals die Kurzform der Ganzkörperentspannung. Die Patienten sollen sich dabei abermals in eine konkrete, reale Situation versetzen. Zu Beginn wird ein letztes Mal unter Anleitung des Therapeuten geübt, bevor der letzte Durchgang völlig eigenständig und nur noch auf sein Startsignal hin erfolgt. Die anschließende Phantasiereise führt die Patienten auf eine Insel. Die Abschlussbesprechung dient abermals der Klärung offener Fragen und der Motivation zum regelmäßigen Üben. Um einen erfolgreichen Alltagstransfer weiterhin zu unterstützen, erhalten die Patienten standardisierte Übungstexte zum Nachlesen. 


\subsection{Fragestellung und Zielsetzung der Dissertation}

Die vorliegende Studie soll folgende Fragestellungen klären und die daraus formulierten Hypothesen auf ihre Gültigkeit hin überprüfen:

1. Wie hoch ist der jeweilige Anteil der Patienten, die im Rahmen des PR-Kurses Entspannungsübungen während des Klinikaufenthalts außerhalb des Kurses durchführten und in welcher Häufigkeit geschah dies? Wie hoch ist der jeweilige Anteil nach optimistischer vs. konservativer Schätzung im Vergleich zur Vorgängerstudie von Arndt (2006)?

Hypothese 1: Im Rahmen der Vorgängerstudie von Arndt (2006) übten 82,5\% der Patienten außerhalb der Übungsstunden mindestens einmal pro Woche (optimistische Schätzung). In der vorliegenden Studie kann im Rahmen des modifizierten Kurses ein ähnlich hoher (nach optimistischer Schätzung) bzw. ein signifikant höherer Anteil (nach konservativer Schätzung) erzielt werden.

2. Wie hoch ist der jeweilige Anteil der Patienten, die im Rahmen des PR-Kurses Entspannungsübungen nach dem Klinikaufenthalt durchführten und wie häufig geschah dies? Wie hoch ist der jeweilige Anteil nach optimistischer vs. konservativer Schätzung im Vergleich zur Vorgängerstudie von Arndt (2006)?

Hypothese 2: Der in der Vorgängerstudie von Arndt (2006) ermittelte Anteil der Patienten mit erfolgreichem Alltagstransfer war 52,5\% nach optimistischer und 31,6\% nach konservativer Schätzung. Im Rahmen der Evaluation des modifizierten Kurses kann ein ähnlich hoher (optimistische Schätzung) bzw. ein signifikant höherer Anteil (konservative Schätzung) erzielt werden.

3. Welche Prädiktoren sagen die Übungshäufigkeit zu Hause voraus?

Hypothese 3: Die Übungshäufigkeit während des Klinikaufenthalts außerhalb der Kursstunden (3a), das veränderte Entspannungserleben nach dem Kurs und nach drei Monaten (3b) als auch die Bewertung der Gruppengröße (3c), die Benotung des Entspannungstrainings (3d), Alter und Geschlecht (3e) und das aktuelle subjektive Wohlbefinden zu T3 (3f) sind wertvolle Prädiktoren eines erfolgreichen Alltagstransfers. 
4. Besteht ein Zusammenhang zwischen der Übungshäufigkeit nach drei Monaten und der Belastung durch anspannungsbezogene Symptome?

Hypothese 4: Die Patienten können einen signifikanten Symptomrückgang nach Abschluss der Rehabilitation und drei Monate nach Entlassung verzeichnen (4a). Sowohl die Beschwerden an sich als auch der Symptomrückgang korrelieren mit der Übungshäufigkeit zuhause (4b). Die Symptome, die Symptomentwicklung und das veränderte Entspannungserleben unterscheiden sich signifikant im Vergleich „Übende“ vs. „Nicht-Übende“ (4c) sowie im Vergleich der extremen Quartile (4d).

Die vorliegende Arbeit möchte einen Beitrag zur Verbesserung des Alltagstransfers von Progressiver Relaxation leisten. Die Evaluation des modifizierten PR-Entspannungstrainings nach einem festgelegten Konzept sowie die Identifikation entsprechender Prädiktoren eines erfolgreichen Alltagstransfers bilden die Grundlage.

Die Pilotstudie von Arndt aus dem Jahre 2005/2006 beinhaltete keine repräsentative Stichprobe, da von insgesamt 316 stationären Aufnahmen lediglich 182 Patienten (57,6\%) sich zur Teilnahme an der Studie bereiterklärten und zum Zeitpunkt T3 nur 80 Patienten (25,3\%) die Befragung abschlossen. Desweiteren war keine genaue Drop-out-Analyse möglich.

Das Ziel der vorliegenden Studie war es daher, einen repräsentativen Anteil der Patientenpopulation in die Studie einzuschließen und eine genaue Drop-out-Analyse zu ermöglichen. 


\section{Material und Methodik}

\subsection{Stichprobe}

Bei der vorliegenden Studie handelt es sich um eine prospektive Längsschnittstudie, die in der Fachklinik für Psychosomatische Medizin der MediClin Bliestal Kliniken Blieskastel im Saarland durchgeführt wurde.

Die Probanden wurden zu drei verschiedenen Zeitpunkten mittels standardisierter Fragebögen befragt: bei Ankunft (T1), bei Abreise (T2) und drei Monate nach Abreise postalisch (T3). Zu Beginn wurde bei 447 Patienten von 469 stationären Aufnahmen die Indikation zur Teilnahme am PR-Kurs gestellt. 411 Patienten (91,9\%) erklärten sich zur Teilnahme an der Studie bereit. Diese nahmen im Rahmen ihres vier- bis sechswöchigen stationären Aufenthalts an dem Kurs „Progressive Relaxation“ teil. 303 Patienten waren weiblichen Geschlechts (73,7\%), 108 Patienten waren Männer (26,3\%). Das Alter lag im Mittel bei 49,14 \pm 8,48 Jahren. Der älteste Patient war 74 Jahre, der jüngste 19 Jahre alt. 360 Patienten wurden jeweils einer der folgenden vier Hauptdiagnosen zugeordnet: Schmerzstörung (43 Probanden), Angststörung (50 Probanden), Depression (119 Probanden) und Anpassungsstörung (148 Probanden). Bei 51 Patienten war eine eindeutige Zuordnung nicht möglich (Welsch, 2010).

Bei der Abreise umfasste die vorliegende Stichprobe 350 Patienten. Einschließendes Kriterium war dabei die Teilnahme an mindestens vier der sechs angebotenen Entspannungssitzungen. Bei geringerer Teilnahmehäufigkeit muss davon ausgegangen werden, dass das Entspannungsverfahren in der Kürze der Zeit nicht ausreichend vermittelt werden kann, um einen langfristigen Übungserfolg zu gewährleisten. Zum Zeitpunkt T2 umfasste die Studie noch 85,2\% der ursprünglich eingeschlossenen Probanden. Drei Monate nach Abreise wurden die Patienten mittels Fragebögen postalisch zu Ihrem Entspannungsverhalten im Alltag befragt (siehe Kapitel 3.2). 274 Probanden schickten die Fragebögen zurück, was einer Rücklaufquote von $\mathbf{7 8 , 3} \%$ entspricht. Eine Übersicht über eingeschlossene Patientenzahlen sowie eine Drop-out-Analyse liefert die Abbildung 2. 


\section{Stationäre Aufnahmen: 469 Patienten}

PR-Indikation: 447 Patienten

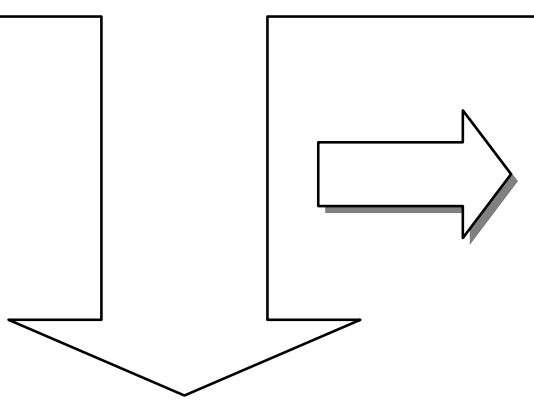

Drop out-Analyse:

Keine Teilnahme an der Studie:

36 Patienten $(8,1 \%)$

T1 (bei Ankunft): 411 Patienten (91,9\%)

$303 w(73,7 \%), 108 m(26,3 \%)$

$\mathrm{MW}($ Alter $)=49,14(\mathrm{SD}=8,48)$

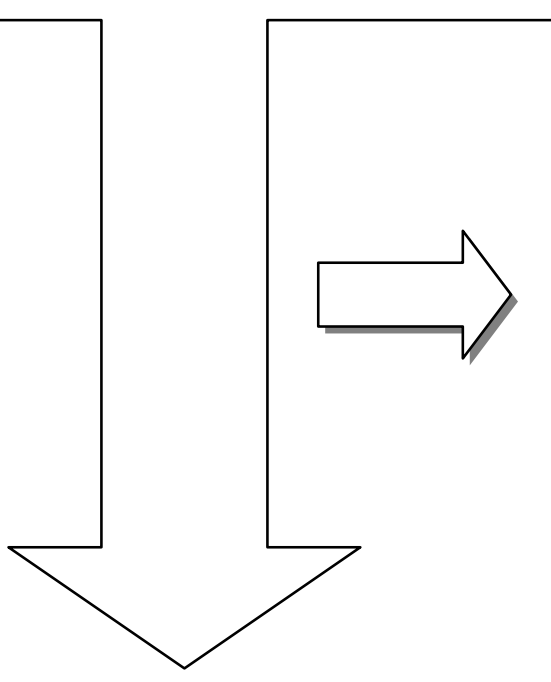

T2 (bei Abreise): 350 Patienten $(\mathbf{8 5 , 2 \% )}$

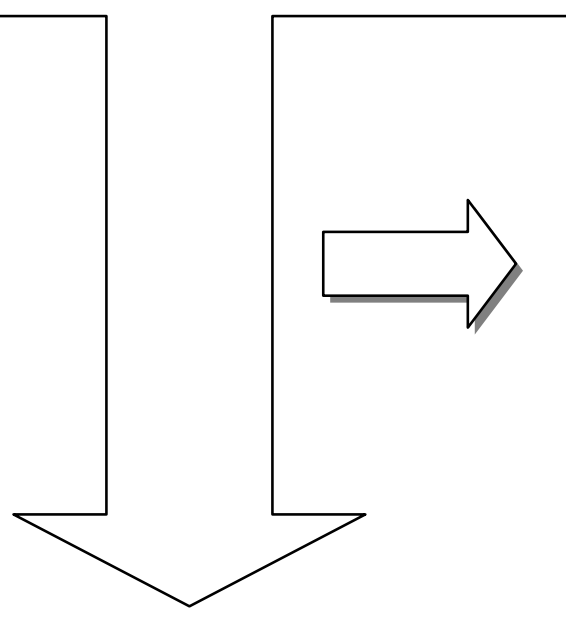

Drop out-Analyse:

Nicht zum Kurs erschienen:

12 Patienten (2,9\%)

$<4$ PR-Sitzungen:

20 Patienten (4,9\%)

Ausstieg aus dem PR-Kurs:

12 Patienten (2,9\%)

Ausstieg aus der Studie:

17 Patienten $(4,1 \%)$

Drop out (T1-T2):

61 Patienten (14,8\%)

Drop out-Analyse:

Offene Teilnahmeverweigerung:

keine

Verstorben:

keine Angaben

Drop out (T2-T3):

76 Patienten $(21,7 \%)$

Drop out (T1-T3) insgesamt:

137 Patienten $(33,3 \%)$

T3 (n. 3 Mo.): 274 Patienten (78,3\%)

Abb.2: Übersicht über Patientenzahlen und Drop out-Analyse 
Im Zeitfenster der Untersuchung (20 Kalenderwochen) wurden insgesamt 469 Patienten zur stationären Behandlung aufgenommen. Davon erklärten sich 411 Patienten zur Teilnahme an der vorliegenden Studie bereit. Bei 22 Patienten (4,7\%) wurde die Indikation zur Teilnahme am Kurs der Progressiven Relaxation nicht gestellt und 36 Patienten (7,7\%) lehnten die Mitarbeit an der Studie ab.

Bei Abreise aus der Klinik mussten insgesamt 61 Patienten $(14,8 \%)$ aus der Studie ausgeschlossen werden. 12 von ihnen (2,9\%) waren nicht zum Kurs erschienen, 20 Patienten $(4,9 \%)$ absolvierten weniger als vier der sechs angebotenen Entspannungssitzungen, $12 \mathrm{~Pa}$ tienten (2,9\%) stiegen aus verschiedenen Gründen (andere Entspannungsalternativen, Schmerzen bei der Durchführung, erfolgreichere Entspannung in Einzelsitzungen) aus dem Kurs der Progressiven Relaxation aus und 17 Patienten $(4,1 \%)$ brachen die Mitarbeit an der Studie ab.

Von den 350 Patienten zum Zeitpunkt T2 schickten 274 Patienten (78,3\%) die nach drei Monaten postalisch versandten Fragebögen zurück. Die 76 Drop-outs $(21,7 \%)$ unterschieden sich hinsichtlich Alter und Geschlecht nicht vom verbleibenden Patientenkollektiv ( $\mathrm{t}=0,461$ resp. t=0,594; n. s.). Kein Brief wurde als unzustellbar an die Klinik zurückversandt. Desweiteren liegen keine Angaben zu bereits verstorbenen Patienten vor. Die Drop-out-Quote betrug im Verlauf von T1 bis T3 insgesamt 33,3\% (137 Patienten), wobei auch hier keine Unterschiede hinsichtlich Alter und Geschlecht im Vergleich zum verbleibenden Patientenkollektiv bestanden ( $t=-1,191$ resp. $t=0,534 ; n$. s. $)$.

\subsection{Aufbau und zeitlicher Rahmen der Untersuchung}

Die vorliegende Studie wurde von März 2009 bis einschließlich Januar 2010 durchgeführt: Mitte März (KW 12) wurden die ersten Probanden eingeschlossen und Mitte Dezember (KW 50) die letzten Fragebögen verschickt. Die KW 4 (Ende Januar 2010) wurde als Endpunkt der Studie mit Annahme der letzten zurückgeschickten Fragebögen definiert.

Folgende Tabelle zeigt den Aufbau der Untersuchung, sowie die Anzahl der Probanden (N) zu T1, T2, T3 im Überblick. 
Tab.9: Übersicht Messzeitpunkte, eingesetzte Messinstrumente u. Anzahl der Probanden (N)

\begin{tabular}{|c|c|c|}
\hline Messzeitpunkte: & Messinstrumente: & $\mathrm{N}:$ \\
\hline - T1 (vor dem Kurs) & $\begin{array}{ll}\text { - } & \text { AT-ANAM } \\
\text { - } & \text { AT-SYM }\end{array}$ & 411 \\
\hline - T1a (nach jeder Sitzung) & - AT-STD & \\
\hline - T2 (nach dem Kurs) & $\begin{array}{ll}\text { - } & \text { AT-VFE } \\
\text { - } & \text { AT-SYM }\end{array}$ & $350(85,2 \%)$ \\
\hline - T3 (drei Monate nach Abreise) & $\begin{array}{l}\text { - } A T-K A T A M \\
\text { - } A T-V F E \\
\text { - } A T-S Y M\end{array}$ & $274(78,3 \%)$ \\
\hline
\end{tabular}

Am Anreisetag wurden die Patienten im Rahmen des allgemeinen Einführungsvortrags über Sinn und Ablauf der Untersuchung informiert. In der ersten Woche des Aufenthalts fand das bereits beschriebene „Grundlagenseminar Entspannung“ statt, in dem die Patienten über Entspannungsverfahren im Allgemeinen, über die Progressive Relaxation im Besonderen, über den Entspannungskurs in Blieskastel sowie über Wirkungen und Nebenwirkungen dieser Entspannungsmethode aufgeklärt wurden. Dieser Termin wurde als T1 definiert und umfasste das Ausfüllen des Anamnese- und Symptomfragebogens.

In der zweiten Woche begann der Entspannungskurs. Am Ende jeder Sitzung (T1a) wurde ein Stundenprotokoll (AT-STD) ausgefüllt, um den Entspannungszustand und etwaige Probleme bei den Übungen, aber auch die Teilnahmehäufigkeit an den Gruppensitzungen zu erfassen. Eine Auswertung dieser Bögen fand innerhalb der vorliegenden Studie nicht statt.

Am Vortag der Abreise (T2) wurde im Rahmen einer Abschiedsveranstaltung der Symptomund Veränderungsfragebogen verteilt und ausgefüllt.

Drei Monate nach Abreise (T3) wurden die Probanden postalisch erreicht und gebeten, den Katamnese-, Veränderungs- und Symptomfragebogen auszufüllen und zurückzuschicken. 


\subsection{Messinstrumente}

Die in der vorliegenden Studie eingesetzten standardisierten Fragebögen gehen auf das „Diagnostische und evaluative Instrumentarium zum Autogenen Training“ von Krampen zurück (AT-EVA, Krampen, 1991). Im Laufe einer 25jährigen Entwicklungsgeschichte entstand auf dieser Grundlage das „Diagnostische und evaluative Instrumentarium für Entspannungstraining und Entspannungstherapie“ (ET-EVA), da sich herausstellte, dass Teile des Instrumentariums AT-EVA in leicht modifizierter Version auch im Rahmen anderer systematisierter Entspannungsverfahren erfolgreich eingesetzt werden konnten (Krampen, 2002a).

Somit entstand ein Testmanual, das interventionsspezifisch zur Differentialdiagnostik und Evaluation eingesetzt werden kann - mit dem Ziel einer spezifischen Indikationsstellung und Effektkontrolle systematisierter Entspannungstherapien. Ausgangspunkte dieses Manuals blieben die von Schultz (1969) postulierten Zielbereiche des Autogenen Trainings (Krampen, 2002a):

- Erholung und Entspannung auf körperlicher und seelischer Ebene

- Innere Ruhe und Lösung zur Selbstentspannung

- Harmonisierung und Regulierung willkürlicher und autonomer Körperfunktionen

- Steigerung von Leistung und Konzentration durch Mobilisierung eigener Ressourcen

- Verminderung der Schmerzwahrnehmung

- Verbesserung der Selbstwirksamkeit (etwa in Krisensituationen)

- Verbesserte Selbstkontrolle durch ausgeprägte Selbstwahrnehmung

Bisher wurden zwei Bestandteile des Instrumentariums für Entspannungstraining und Entspannungstherapie (ET-EVA) als Testmanuale publiziert: ET-ANAM (Krampen 2002a) und ASS-SYM (Krampen 2006). Die übrigen standardisierten Fragebögen wurden mit Genehmigung des Autors und in Kooperation mit diesem modifiziert und eingesetzt. In Anlehnung an das erste standardisierte Instrumentarium (AT-EVA) wurden die Messinstrumente dementsprechend benannt.

Vor der anonymisierten Datenerhebung lag eine unterschriebene Einverständniserklärung vor.

Die entsprechenden Fragebögen wurden mit einer Forschungsnummer versehen, die streng getrennt von den persönlichen Daten aufbewahrt wurde. 


\subsubsection{Anamnesefragebogen (AT-ANAM)}

Der erste Fragenkomplex beinhaltet Fragen zu individuellen Vorerfahrungen mit anderen Entspannungsverfahren (Autogenes Training, Progressive Relaxation, Yoga oder andere Meditations- und Entspannungsmethoden) sowie deren Anwendungs- und Lernmodus (Einzel- oder Gruppensitzung). Weiterhin werden die Patienten gebeten, den ungefähren Zeitpunkt des Erlernens sowie die aktuelle Anwendungshäufigkeit anzugeben.

Die Fragen fünf bis acht erfassen mögliche absolute oder relative Kontraindikationen durch Erfragung bestehender oder überstandener Herz-Kreislauf-Erkrankungen, psychiatrischer, neurologischer oder chronischer Krankheiten und des aktuellen Medikamentenkonsums.

Im letzen Abschnitt des vier Seiten umfassenden Fragebogens werden die Teilnahmemotive des Kursteilnehmers ermittelt. Der Proband hat die Möglichkeit, unter 16 vorgegebenen Teilnahmemotiven (mehrere) zu wählen oder im Rahmen freier Zeilen eigene Motive einzutragen bzw. zu ergänzen. Motivationslage und Erwartungshaltung können somit erfasst werden. Die strukturierte Liste der vorgegebenen Motive basiert sowohl auf dem Wunsch nach adaptierter und selektiver Indikationsstellung als auch auf empirischen Studien zu Prädiktoren eines erfolgreichen Entspannungstrainings, in denen Teilnahmemotive und Vorerfahrungen als wichtige Faktoren identifiziert wurden (Krampen 2002b, 2004; Krampen und von Eye, 2006; Bernardy et al., 2008).

\subsubsection{Symptomfragebogen (AT-SYM)}

Der Symptomfragebogen beinhaltet Fragen nach subjektiver Beschwerdebelastung und erfasst sechs Indikationsbereiche auf sechs Subskalen mit jeweils acht Items:

1. Körperliche und psychische Erschöpfung

2. Nervosität und innere Anspannung

3. Psychophysiologische Dysregulation

4. Leistungs- und Verhaltensschwierigkeiten

5. Schmerzbelastung

6. Probleme mit Selbstbestimmung und Selbstkontrolle 
Dieser Fragebogen wurde vor Beginn des Kurses, nach Abschluss sowie drei Monate nach Entlassung im Rahmen des Katamneseschreibens eingesetzt. Er dient somit der indirekten ergebnisorientierten Veränderungsmessung (Krampen, 2002a).

\subsubsection{Stundenfragebogen (AT-STD)}

Der Protokollbogen zum Ende jeder Entspannungssitzung dient der ökonomischen Prozessevaluation (Krampen, 2002a). Im Rahmen der vorliegenden Studie lag diesbezüglich der Schwerpunkt auf der Erfassung etwaiger Probleme bei den Entspannungsübungen sowie der Teilnahmehäufigkeit (Teilnahme an vier von sechs Sitzungen als Einschlusskriterium). Die ersten fünf Fragen des AT-Stundenbogens wurden übernommen und erfassen allgemeine Einschätzungen zum individuellen Entspannungszustand und zum Wohlbefinden in der Gruppe bzw. der wahrgenommenen Gruppenatmosphäre (Krampen, 2002a). Die Fragen sechs bis zwölf des ursprünglichen AT-Stundenbogens, die sich auf die sieben AT-Vorsatzformeln beziehen, wurden durch Fragen zur subjektiv erlebten Entspannung der einzelnen Muskelgruppen ersetzt.

\subsubsection{Veränderungsfragebogen (AT-VFE)}

Der Veränderungsfragebogen erfasst, ob und inwieweit die Probanden im Verlauf der Teilnahme und im Hinblick auf den Kurs eine negative, keine oder eine positive Veränderung ihres Entspannungserlebens und Wohlbefindens verzeichnen können. Die Stärke der Veränderung variiert auf einer Skala von 1 (starke negative Veränderung) bis 7 (starke positive Veränderung). Jeweils zwei Items der insgesamt zwölf Fragen beziehen sich auf die sechs Indikationsbereiche des Symptomfragebogens (zwei Fragen zur veränderten Fähigkeit zur körperlichen und seelischen Entspannung, zwei Fragen zur inneren Lösbarkeit, zur Selbstregulation von Körperfunktionen, zu Medikamenteneinnahme und Schlafverhalten, zwei Fragen zur Leistungssteigerung und abschließend zur veränderten Fähigkeit zur Selbstkontrolle). Somit ist es möglich, die indirekte Veränderungsmessung des Symptombogens durch eine direkte Veränderungsmessung zu ergänzen. 
Sechs Fragen zur Übungshäufigkeit außerhalb des Kurses während des Klinikaufenthalts, zum Hilfsmaterialienangebot und dem Wunsch nach Anleitungen, zur Gruppengröße und zu Verbesserungsvorschlägen vervollständigen diesen Fragenkomplex. Dieser Veränderungsbogen wurde zum Zeitpunkt T2 und im Rahmen des Katamneseschreibens eingesetzt.

\subsubsection{Katamnesefragebogen (AT-KATAM)}

Mittels Katamnesefragebogen werden die aktuelle Übungshäufigkeit, die Bewertung der Entspannungsmethode und das aktuelle persönliche Befinden erfragt. Die bevorzugte Körperhaltung während des Trainings und die Wirkung der Übungen auf bestimmte Körperregionen - sofern das Entspannungstraining noch angewandt wird - können angegeben werden.

Darüber hinaus wird der Fragebogen durch einen speziellen Fragenkomplex zum Entspannungstraining in Blieskastel ergänzt. Dieser umfasst eine Beurteilung des Grundlagenseminars, des Kurses, des Kursleiters und der Therapie im Allgemeinen - jeweils auf einer Notenskala von 1 (sehr gut) bis 6 (ungenügend). Außerdem werden die Probanden zum Verhalten des Kursleiters (Ermutigung zum eigenständigen Üben und zum Erfahrungsaustausch), zu Materialen und zur Gruppengröße befragt. Abschließend bieten freie Zeilen Platz für individuelle Anmerkungen.

\subsection{Auswertung der Daten}

Die ausgehändigten Fragebögen lagen in maschinell lesbarer Form vor und konnten mittels Scanner erfasst und digitalisiert werden. Die statistische Auswertung erfolgte computergestützt mittels des Programms SPSS (Superior Performing Statistical Software) Version 17 für Microsoft Windows. Insgesamt wurden 367 Variablen im Rahmen von 2344 Fragebögen erfasst. Dabei wurden die String-Variablen mit Freitext von Hand nachgetragen. Da die Fragebögen nahezu vollständig beantwortet waren, konnte die Anzahl der Missing Items (unbeantwortete Fragen) vernachlässigt werden.

Die Ergebnisse wurden mit denen der Vorgängerstudie von Arndt (2006) hinsichtlich absoluter und relativer Häufigkeiten (optimistische vs. konservative Schätzung) im Rahmen des t-Tests für unabhängige Stichproben verglichen. Mittels Levene-Test wurde eine mög- 
liche Homogenität der Varianzen ermittelt. Bei ungleichen Varianzen fand der Welch-Test Anwendung.

Als Maß der Veränderung der Symptome wurden Differenzwerte der Skalenmittelwerte zwischen den Messzeitpunkten berechnet. Anhand des t-Tests für verbundene Stichproben konnte ein Mittelwertvergleich der Skalen des Symptomfragebogens durchgeführt und somit die Entwicklung der Beschwerden zu drei verschiedenen Zeitpunkten (T1, T2, T3) erfasst werden. Effektstärken wurden nach der Formel für abhängige Stichproben von Dunlap et al. (1996) bestimmt, die die Korrelation der beiden Messwerte berücksichtigt. Nach Cohen (1988) kann die Größe eines Effekts von 0,2 bis <0,5 als klein, von 0,5 bis <0,8 als mittel und $\geq 0,8$ als groß eingeschätzt werden.

Um die Veränderung des Entspannungserleben und Wohlbefindens nach dem PR-Kurs und drei Monate nach Entlassung quantifizieren zu können, wurden ebenfalls Mittelwerte der 12 Items des Veränderungsfragebogens gebildet. Zudem wurden verschiedene Gruppen innerhalb des Patientenkollektivs gebildet und diese hinsichtlich anspannungsbezogener Symptombelastung zu T3, Symptomentwicklung und verändertem Entspannungserleben gegenüber gestellt. Die folgende Tabelle liefert eine Übersicht über die verschiedenen Gruppen, deren definierter Übungshäufigkeit sowie die Anzahl der entsprechenden Studienteilnehmer.

Tab.10: Gruppen innerhalb des Patientenkollektivs, definierte Übungshäufigkeit und Anzahl der Probanden (N)

\begin{tabular}{|c|c|c|}
\hline $\begin{array}{l}\text { Gruppen innerhalb des Patien- } \\
\text { tenkollektivs: }\end{array}$ & Definierte Übungshäufigkeit: & $\mathrm{N}:$ \\
\hline $\begin{array}{l}\text { Übende insgesamt: } \\
\text { - } \quad \text { Häufig Übende } \\
\text { - } \quad \text { Regelmäßig Übende }\end{array}$ & $\begin{array}{l}\text { Mindestens einmal pro Woche } \\
\text { - Einmal bis mehrmals am Tag } \\
\text { - Einmal bis mehrmals pro Woche }\end{array}$ & $\begin{array}{l}138 \\
43 \\
95\end{array}$ \\
\hline $\begin{array}{l}\text { Nicht-Übende insgesamt: } \\
\text { - Selten Übende } \\
\text { - Nicht Übende }\end{array}$ & $\begin{array}{l}\text { Weniger als einmal pro Woche } \\
\begin{array}{l}\text { - Selten bis seit dem Einführungs- } \\
\text { kurs nicht (mehr) } \\
\text { - Einmal bis mehrmals pro Monat }\end{array}\end{array}$ & $\begin{array}{l}136 \\
75\end{array}$ \\
\hline
\end{tabular}

Um Prädiktoren eines erfolgreichen Alltagstransfers zu identifizieren, wurden die Übungshäufigkeit während des Reha-Aufenthalts, das veränderte Entspannungserleben nach dem PR- 
Kurs und drei Monate nach Entlassung, die Belastung durch anspannungsbezogene Symptome bzw. deren Entwicklung im Verlauf der Erhebung als auch die Bewertung der Gruppengröße, die Benotung des Entspannungskurses, Alter und Geschlecht sowie das subjektiv eingeschätzte Wohlbefinden zu T3 mit der Übungshäufigkeit im Alltag korreliert. Dabei wurden bivariate Korrelationen nach Spearman-Rho mit einem Signifikanzniveau von $\mathrm{p}<0,05$ bzw. p $<0,01$ berechnet. Signifikante Ergebnisse liegen bei einer Irrtumswahrscheinlichkeit von maximal 5\% vor $(\mathrm{p}<0,05)$, bei einer Irrtumswahrscheinlichkeit von maximal $1 \%(\mathrm{p}<0,01)$ wird von einem hochsignifikanten Ergebnis ausgegangen.

Um herauszufinden, durch welche als signifikant identifizierte Prädiktoren die Übungshäufigkeit im Alltag hauptsächlich erklärt werden kann, wurde abschließend eine lineare Regressionsanalyse durchgeführt. 


\section{Ergebnisse}

\section{1. Übungshäufigkeit während des Klinikaufenthalts außerhalb des PR-Kurses}

\subsection{1. Übungshäufigkeit im Rahmen des modifizierten PR-Kurses}

Im Rahmen des modifizierten Entspannungskurses sollen die Patienten regelmäßig durch gezielte Motivationsförderung zum selbstständigen Üben außerhalb der Kursstunden angeleitet werden. Bei Abreise (T2) wurden die verbleibenden 350 Patienten gebeten, ihre Übungshäufigkeit während des Reha-Aufenthaltes anzugeben. Die Ergebnisse stellt die folgende Abbildung zusammen.

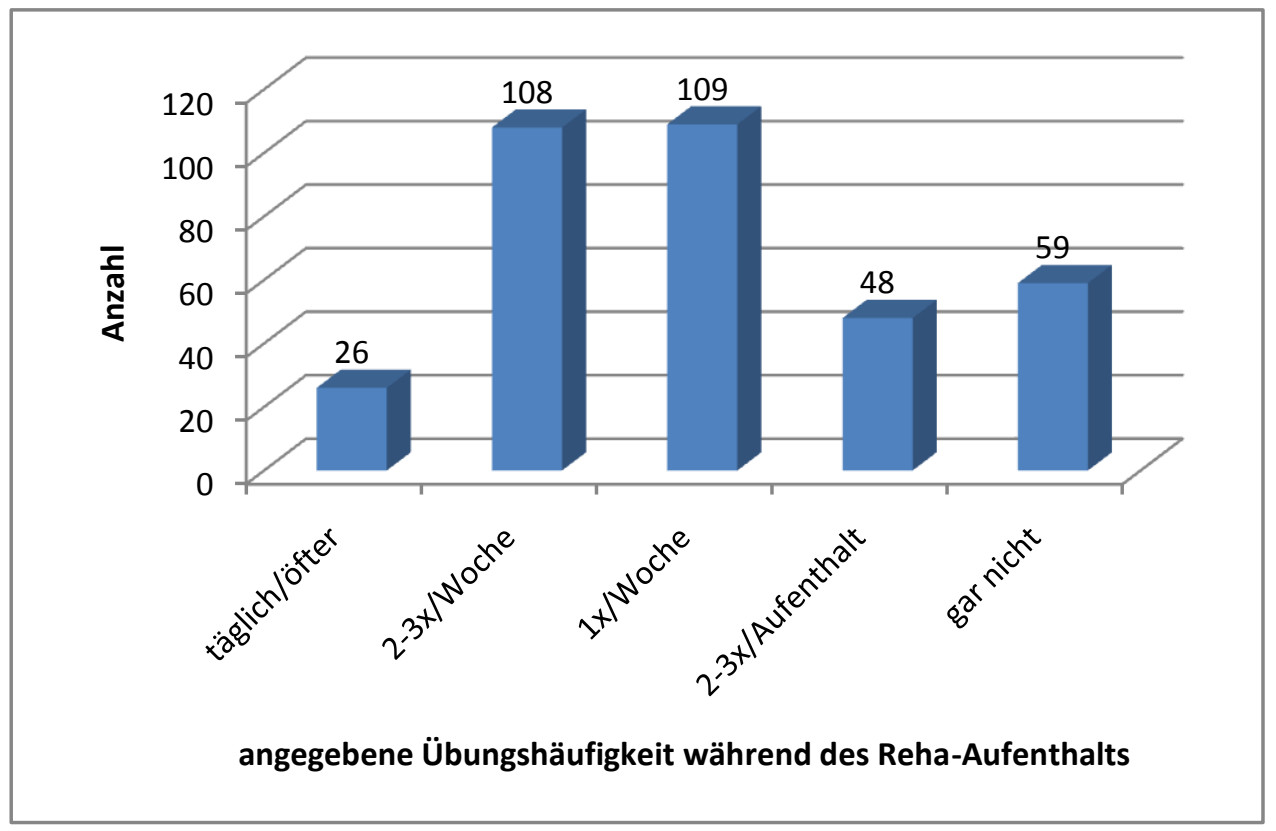

Abb.3: Häufigkeitsangaben zum selbstständigen Üben zu T2 - MW=3,02; SD=1,19; N=350

350 Patienten gaben ihre Übungshäufigkeit während des Reha-Aufenthalts an. Die mittlere Übungshäufigkeit lag bei 3,02 (SD=1,19), d. h. durchschnittlich übten die Patienten ungefähr einmal pro Woche; 38,3\% übten häufiger, 30,6\% seltener. Als Zielkriterium einer erfolgreichen selbstständigen Übungsroutine wurde die Übungshäufigkeit von mindestens einmal pro Woche definiert. Zusammenfassend konnten somit 69,4\% der Patienten (243 Patienten) die Entspannungsübungen erfolgreich in ihren Klinikalltag integrieren (nach optimistischer Schätzung 243 von 274 Patienten zum Zeitpunkt T3, also 88,7\%). 
Motivation zum selbstständigen Üben und Erfahrungsaustausch. Die Patienten wurden im Rahmen des Katamnesefragebogens befragt, wie häufig sie der Kursleiter zum selbstständigen Üben aufforderte und inwieweit die Möglichkeit bestand, im Kurs über die Erfahrungen beim selbstständigen Üben zu sprechen. Diese Fragen wurden von allen verbliebenen Studienteilnehmern zu T3 beantwortet (N=274). Die Auswertung der angegebenen Häufigkeiten ergab, dass die Kursteilnehmer regelmäßig zum selbstständigen Üben motiviert wurden (MW=1,28; $\mathrm{SD}=0,71)$ und dass regelmäßig die Gelegenheit zum Erfahrungsaustausch bestand $(\mathrm{MW}=1,45 ; \mathrm{SD}=0,75)$.

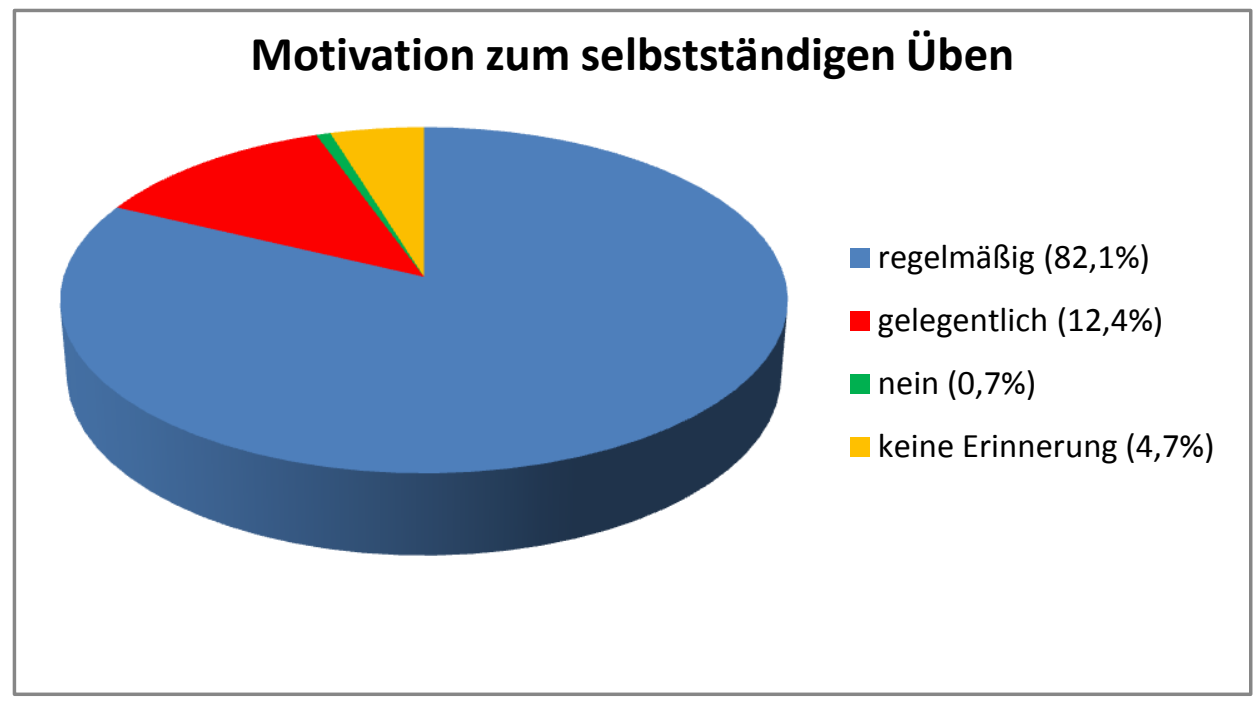

Abb.4: Motivation zum selbstständigen Üben durch den Kursleiter

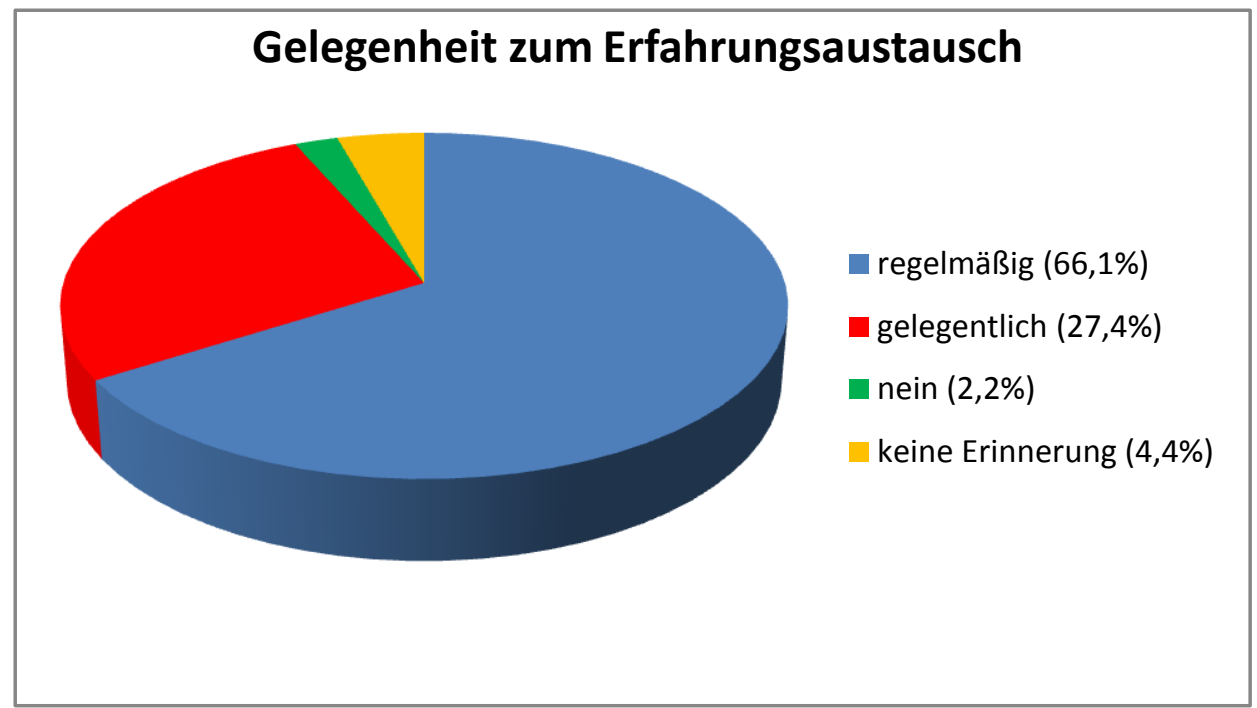

Abb.5: Gelegenheit zum Austausch von eigenen Erfahrungen beim selbstständigen Üben 


\subsubsection{Vergleich mit Ergebnissen der Vorläuferstudie}

Auch im Rahmen der Vorläuferstudie von Arndt (2006) wurden die Studienteilnehmer bei Entlassung zu ihrer Übungshäufigkeit außerhalb des Entspannungskurses befragt. Das Patientenkollektiv der Pilotstudie (N=80) übte im Mittel 1-2x/Woche (MW=2,59; $\mathrm{SD}=1,12)$. Insgesamt konnten nach optimistischer Schätzung 66 von insgesamt 80 Teilnehmern (82,5\%), die die Fragebögen zu allen drei Messzeitpunkten ausfüllten, die Entspannungsübungen erfolgreich in ihren Klinikalltag integrieren. Setzt man diese 66 erfolgreichen Studienteilnehmer ins Verhältnis zu den 133 Patienten, die den Entspannungskurs absolvierten, ist nach konservativer Schätzung von einer „Erfolgsquote“ von $\mathbf{4 9 , 6 \%}$ auszugehen. Folgende Tabelle stellt die absoluten und relativen Häufigkeiten der beiden zu vergleichenden Studien gegenüber.

Tab.11: Vergleich der beiden Studien hinsichtlich Übungshäufigkeit in der Klinik

\begin{tabular}{|l|l|l|l|}
\hline $\begin{array}{l}\text { Übungshäufigkeit in der } \\
\text { Reha }\end{array}$ & Arndt (2006) & Klosterhalfen (2010) & Sign. (2-seitig) \\
\hline Täglich/öfter (1) & $10 \%$ (8 Pat.) & $7,4 \%$ (26 Pat.) & \\
\hline $2-3 x /$ Woche (2) & $50 \%$ (40 Pat.) & $30,9 \%$ (108 Pat.) & \\
\hline $1 x /$ Woche (3) & $22,5 \%$ (18 Pat.) & $31,1 \%$ (109 Pat.) & \\
\hline $2-3 x /$ Aufenthalt (4) & $6,3 \%(5$ Pat.) & $13,7 \%$ (48 Pat.) & \\
\hline Gar nicht (5) & $11,3 \%$ (9 Pat.) & $16,9 \%(59$ Pat.) & \\
\hline Optimistische Schätzung & $82,5 \% ; N=80$ & $88,7 \% ; N=274$ & $t=1,320 ; n . s$. \\
\hline Konservative Schätzung & $49,6 \% ; N=133$ & $69,4 \% ; N=350$ & $t=3,959 ; p<0,01$ \\
\hline
\end{tabular}

Anhand des t-Tests für unabhängige Stichproben wurden die relativen Häufigkeiten nach optimistischer und konservativer Schätzung verglichen. Mittels Levene-Test wurde die Gleichheit der Varianzen ausgeschlossen; der Welch-Test fand Anwendung.

Nach konservativer Schätzung konnte ein signifikant höherer Anteil der Studienteilnehmer von 2010 die erlernten PR-Übungen in den Klinikalltag integrieren $(t=3,959 ; p<0,01)$. 


\subsubsection{Prädiktoren eines erfolgreichen Kliniktransfers}

Um mögliche Prädiktoren einer erfolgreichen Übungsroutine während des Klinikaufenthalts zu identifizieren, wurde die Übungshäufigkeit in der Klinik mit den Variablen ,,verändertes Entspannungserleben zu T2“ sowie mit der Symptombelastung zu T1 korreliert (SpearmanRho). Die folgende Tabelle fasst die Ergebnisse zusammen.

Tab.12: Mögliche Prädiktoren eines erfolgreichen Kliniktransfers

\begin{tabular}{|l|l|}
\hline Variablen & Übungshäufigkeit in der Klinik \\
\hline Verändertes Entspannungserleben (T2) & $r=-0,184 ; p<0,01$ \\
\hline Körperliche u. psychische Erschöpfung (T1) & $r=-0,131 ; p<0,05$ \\
\hline Nervosität u. innere Anspannung (T1) & $r=-0,039 ; n . s$. \\
\hline Psychophysiologische Dysregulation (T1) & $r=-0,119 ; p<0,05$ \\
\hline Leistungs- u. Verhaltensschwierigkeiten (T1) & $r=-0,002 ; n . s$. \\
\hline Schmerzbelastung (T1) & $r=-0,166 ; p<0,01$ \\
\hline Selbstbestimmung u. Selbstkontrolle (T1) & $r=-0,043 ; n . s$. \\
\hline
\end{tabular}

Es fanden sich aufgrund der hohen Fallzahl zwar signifikante, jedoch nur sehr schwach ausgeprägte Zusammenhänge: Demnach übten jene Patienten während des Klinikaufenthalts häufiger, die bereits im Laufe des Kurses eine Verbesserung ihres Entspannungserlebens bemerkten und/ oder zu Beginn ihres Reha-Aufenthalts (auf bestimmten Skalen) besonders belastet waren. 


\section{2. Übungshäufigkeit im Alltag nach dem Klinikaufenthalt}

\subsubsection{Erfolgreicher Alltagstransfer im Rahmen des modifizierten PR-Kurses}

Bezüglich der PR-Übungshäufigkeit im Alltag liegen die Angaben von allen 274 Patienten vor, die die Fragebögen ausgefüllt zurückschickten. Die folgende Abbildung liefert eine Übersicht der Ergebnisse.

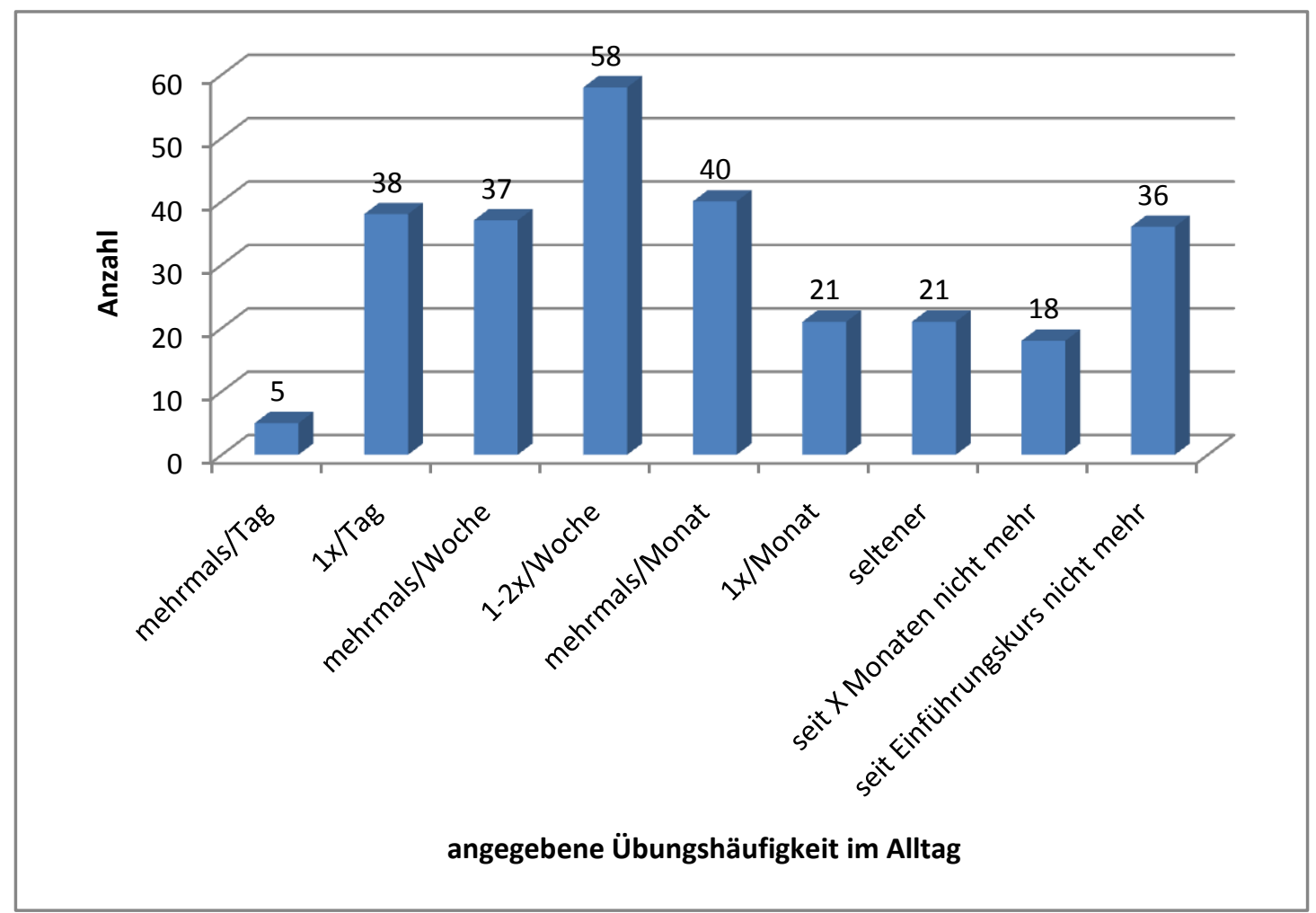

Abb.6: Häufigkeitsangaben zum Üben im Alltag (T3) - MW=4,98; SD=2,32; N=274

Bei einem Mittelwert von 4,98 übten die Patienten im Durchschnitt mehrmals im Monat. Insgesamt konnten 50,4\% der Patienten (138 Studienteilnehmer) zu T3 die Entspannungsmethode erfolgreich in ihren Alltag integrieren, was einer Übungshäufigkeit von mindestens einmal pro Woche entspricht (optimistische Schätzung). Setzt man die 138 erfolgreichen Probanden ins Verhältnis zu allen Patienten, die den Entspannungskurs zum Zeitpunkt T2 absolvierten $(\mathrm{N}=350)$, erhält man nach konservativer Schätzung einen Anteil von 39,4\% der Patienten mit einer Übungshäufigkeit von mindestens einmal pro Woche. 


\subsubsection{Vergleich mit Ergebnissen der Vorläuferstudie}

Arndt konnte im Jahre 2006 einen Anteil von 52,5\% der Patienten mit erfolgreichem Alltagstransfer verzeichnen - 42 der insgesamt 80 Patienten zu T3 übten mindestens einmal pro Woche (optimistische Schätzung). Geht man von der Annahme aus, dass diejenigen Patienten, die das Entspannungstraining absolvierten und zu T3 jedoch nicht antworteten, am Transfer des Gelernten in den Alltag scheiterten, erhält man nach konservativer Schätzung einen Anteil von 31,6\% der Studienteilnehmer mit erfolgreichem Alltagstransfer (nämlich 42 von 133 zu T2). Ein Patient machte zu T3 keine Angaben zu seiner Übungshäufigkeit. Die folgende Tabelle stellt die beiden zu vergleichenden Studien gegenüber.

Tab.13: Vergleich der beiden Studien hinsichtlich Übungshäufigkeit im Alltag

\begin{tabular}{|l|l|l|l|}
\hline Übungshäufigkeit im Alltag & Arndt (2006) & Klosterhalfen (2010) & Sign. (2-seitig) \\
\hline Mindestens 1x/Tag (1/2) & $15 \%$ (12 Pat.) & $15,7 \%$ (43 Pat.) & \\
\hline Mindestens 1-2x/Woche (3/4) & $37,5 \%$ (30 Pat.) & $34,7 \%$ (95 Pat.) & \\
\hline Mindestens 1x/Monat (5/6) & $13,8 \%$ (11 Pat.) & $22,3 \%(61$ Pat.) & \\
\hline Seltener (7) & $16,3 \%(13$ Pat.) & $7,7 \%(21$ Pat.) & \\
\hline Gar nicht (mehr)(8/9) & $16,3 \%(13$ Pat.) & $19,7 \%(54$ Pat.) & \\
\hline Optimistische Schätzung & $52,5 \% ; N=80$ & $50,4 \% ; N=274$ & $t=-0,335 ;$ n. s. \\
\hline Konservative Schätzung & $31,6 \% ; N=133$ & $39,4 \% ; N=350$ & $t=1,629 ; n . s$. \\
\hline
\end{tabular}

Auch im Rahmen der Arndt-Studie übten die Patienten im Mittel etwa mehrmals im Monat ( $M W=4,91, S D=2,40, N=79$ ). Der Vergleich der relativen Häufigkeiten (t-Test für unabhängige Stichproben) erbrachte kein signifikantes Ergebnis. 


\subsection{Prädiktoren eines erfolgreichen Alltagstransfers}

Als mögliche Prädiktoren wurden folgende Angaben mit der alltäglichen Übungsroutine korreliert (bivariate Korrelationen nach Spearman-Rho):

- Übungshäufigkeit während des Klinikaufenthalts außerhalb der Kursstunden

- Verändertes Entspannungserleben zu T2 und T3

- Bewertung der Gruppengröße

- Benotung des Entspannungskurses

- Alter und Geschlecht der Patienten

- Aktuelles subjektives Wohlbefinden zu T3

Die Ergebnisse der bivariaten Korrelation stellt die folgende Tabelle zusammen.

Tab.14: Mögliche Prädiktoren eines erfolgreichen Alltagstransfers

\begin{tabular}{|l|l|}
\hline Variablen & Übungshäufigkeit zu T3 \\
\hline $\begin{array}{l}\text { Übungshäufigkeit während des Klinikaufenthalts außer- } \\
\text { halb der Kursstunden }\end{array}$ & $r=+0,558 ; p<0,01$ \\
\hline Verändertes Entspannungserleben zu T2 & $r=-0,240 ; p<0,01$ \\
\hline Verändertes Entspannungserleben zu T3 & $r=-0,356 ; p<0,01$ \\
\hline Bewertung der Gruppengröße & $r=-0,056 ; n . s$. \\
\hline Benotung des Entspannungskurses & $r=+0,243 ; p<0,01$ \\
\hline Alter der Patienten & $r=-0,127 ; p<0,05$ \\
\hline Geschlecht der Patienten & $r=-0,004 ; n . s$. \\
\hline Aktuelles subjektives Wohlbefinden zu T3 & $r=-0,165 ; p<0,01$ \\
\hline
\end{tabular}

Übungshäufigkeit während des Klinikaufenthalts. Es zeigte sich eine mittelgradige Korrelation zwischen der Übungshäufigkeit im Alltag und der Übungshäufigkeit während des Klinikaufenthalts $(\mathbf{r}=+\mathbf{0 , 5 5 8} ; \mathrm{p}<0,01)$. Diejenigen Patienten, die bereits während des Klinik- 
aufenthalts erfolgreich übten, konnten auch im Alltag die Entspannungsübungen erfolgreich umsetzen.

Verändertes Entspannungserleben. Das Entspannungserleben und Wohlbefinden zeigte bei Abreise (T2) eine positive Veränderung (Mittelwert der 12 Items zu T2=5,044; SD=0,98; $\mathrm{N}=349$ ), die auch nach drei Monaten in abgeschwächter Form noch nachweisbar war (Mittelwert der 12 Items zu T3=4,79; SD=1,1; N=262). Eine Übersicht über die Mittelwerte der einzelnen Items zu T2 und T3 liefert die Tabelle 15.

Die stärksten Veränderungen - sowohl bei Abreise als auch nach drei Monaten - waren auf den Skalen „Fähigkeit zur körperlichen Entspannung“, „Fähigkeit zur seelischen Erholung“, „Wohlbefinden insgesamt“ und „Fähigkeit zur inneren Gelassenheit“" ersichtlich. Die unterschiedliche Anzahl (N) ergibt sich daraus, dass nicht alle Fragen von allen Patienten beantwortet wurden.

Es zeigte sich eine gering- bis mittelgradige Korrelation der Mittelwerte der 12 Items mit der Übungshäufigkeit im Alltag nach drei Monaten (r(T2)=-0,240; r(T3)=-0,356; p<0,01). Demnach war der Alltagstransfer bei jenen Patienten am erfolgreichsten, die die stärkste Verbesserung ihres Entspannungserlebens und Wohlbefindens verzeichnen konnten.

Bewertung der Gruppengröße. Die durchschnittliche Gruppengröße lag zwischen acht und zwölf Personen, wobei auch „Ausreißergruppen“ von fünf und bis zu 25 Personen vorkamen. Sie konnte als „,viel zu groß“, „etwas zu groß“, ,genau angemessen“, „etwas zu klein“ und „,viel zu klein“ bezeichnet werden. Die durchschnittliche Bewertung lag zwischen „etwas zu groß“ und ,angemessen“ (MW=2,66; $\mathrm{SD}=0,62 ;$ Varianz=0,380).

Da diese bipolare Skala mit einer unipolaren Skala (nämlich der Übungshäufigkeit nach drei Monaten) korreliert werden sollte, wurde eine Umkodierung der Variablen vorgenommen. Die Angaben „,viel zu groß“ und „,viel zu klein““ wurden zum Wert 1=,Gruppengröße stark verbesserungswürdig“, die Angaben „etwas zu groß“ und „etwas zu klein“ zum Wert 2=,,Gruppengröße verbesserungswürdig“ zusammengefasst. Die Bewertung 3=,Gruppengröße angemessen“" wurde beibehalten. Die durchschnittliche Bewertung lag auf dieser unipolaren Skala bei 2,64 (SD=0,61; Varianz=0,368). Die folgenden Abbildungen (Abb. 7 und 8) veranschaulichen die angegebenen Häufigkeiten nach bi- und unipolarer Skalenausrichtung.

Die Korrelation der Übungshäufigkeit nach drei Monaten mit der Bewertung der Gruppengröße (unipolar) erbrachte kein signifikantes Ergebnis. 
Tab.15: Übersicht der Mittelwerte der Skalen des AT-VFE zu T2 und T3 (1=,,starke negative Veränderung“ bis 7=,,starke positive Veränderung“)

\begin{tabular}{|c|c|c|}
\hline & $\mathrm{T} 2(\mathrm{MW})$ & T3 (MW) \\
\hline $\begin{array}{l}\text { Fähigkeit zur körperlichen } \\
\text { Entspannung }\end{array}$ & 5,$30 ; S D=1,16 ; N=350$ & 5,$00 ; S D=1,23 ; N=263$ \\
\hline $\begin{array}{l}\text { Fähigkeit zur seelischen Er- } \\
\text { holung }\end{array}$ & 5,$20 ; S D=1,18 ; N=346$ & 4,$91 ; S D=1,23 ; N=264$ \\
\hline $\begin{array}{l}\text { Gesundheitliches Wohlbe- } \\
\text { finden }\end{array}$ & 5,$13 ; S D=1,25 ; N=349$ & 4,$77 ; S D=1,37 ; N=262$ \\
\hline Wohlbefinden insgesamt & 5,$38 ; S D=1,25 ; N=350$ & 4,$93 ; S D=1,31 ; N=262$ \\
\hline Zukunftssorgen & 4,$89 ; \mathrm{SD}=1,36 ; N=349$ & 4,$55 ; S D=1,49 ; N=262$ \\
\hline $\begin{array}{l}\text { Fähigkeit zur inneren Gelas- } \\
\text { senheit }\end{array}$ & 5,$26 ; S D=1,29 ; N=347$ & 4,$92 ; S D=1,32 ; N=262$ \\
\hline Schlafverhalten & 4,$84 ; S D=1,43 ; N=349$ & 4,$75 ; S D=1,46 ; N=262$ \\
\hline Medikamenteneinnahme & 4,$50 ; S D=1,68 ; N=324$ & 4,$51 ; S D=1,66 ; N=235$ \\
\hline Steigerung der Ausdauer & 5,$16 ; S D=1,22 ; N=349$ & 4,$74 ; S D=1,25 ; N=261$ \\
\hline $\begin{array}{l}\text { Steigerung des Konzentra- } \\
\text { tionsvermögens }\end{array}$ & 4,$92 ; S D=1,21 ; N=348$ & 4,$75 ; S D=1,29 ; N=260$ \\
\hline $\begin{array}{l}\text { Fähigkeit zur Gelassenheit } \\
\text { gegenüber Unerwartetem }\end{array}$ & 4,$88 ; S D=1,25 ; N=345$ & 4,$74 ; S D=1,34 ; N=261$ \\
\hline $\begin{array}{l}\text { Fähigkeit zur Selbstkontrolle } \\
\text { gegenüber Stress und } \\
\text { Belastungen }\end{array}$ & 4,$99 ; S D=1,29 ; N=347$ & 4,$80 ; S D=1,30 ; N=264$ \\
\hline Mittelwert aller 12 Items & 5,$044 ; S D=0,98 ; N=349$ & 4,$79 ; S D=1,1 ; N=262$ \\
\hline
\end{tabular}




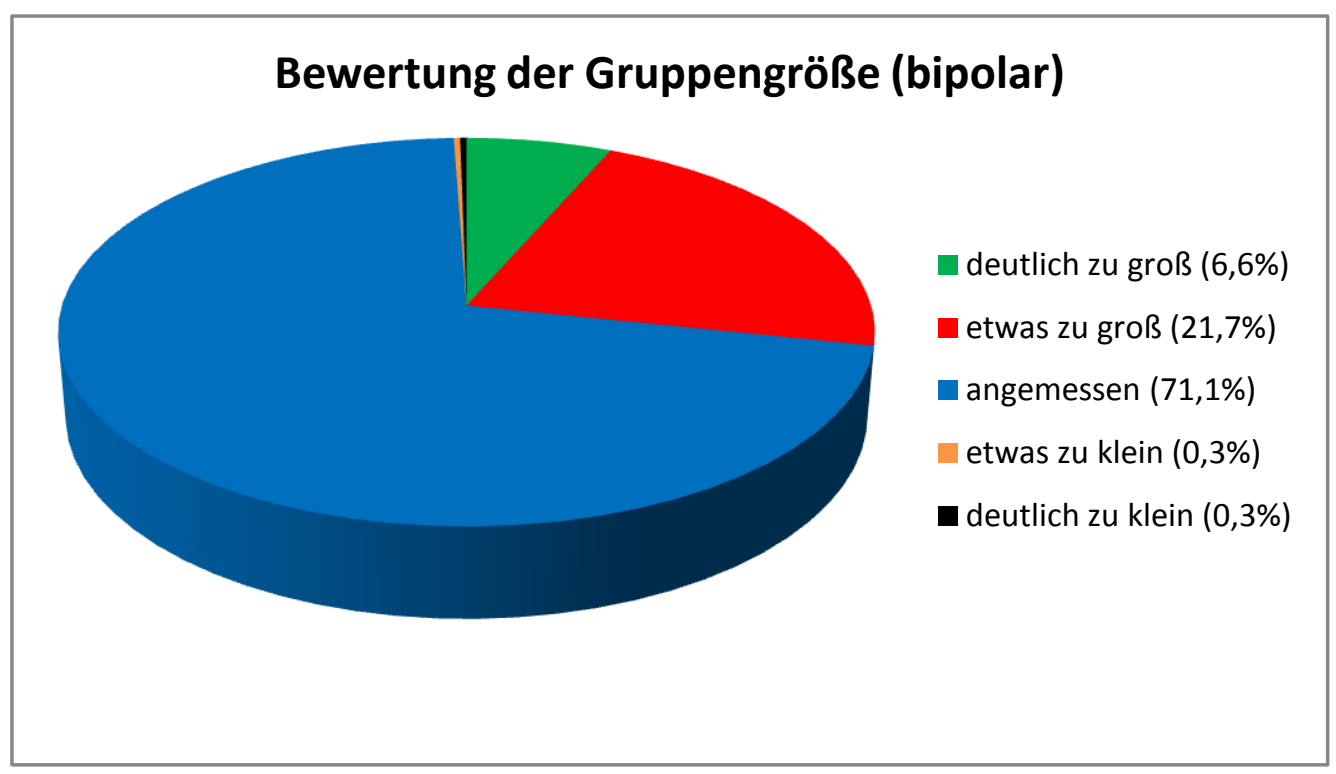

Abb.7: Bewertung der Gruppengröße auf bipolarer Skala (MW=2,66; SD=0,62; N=350)

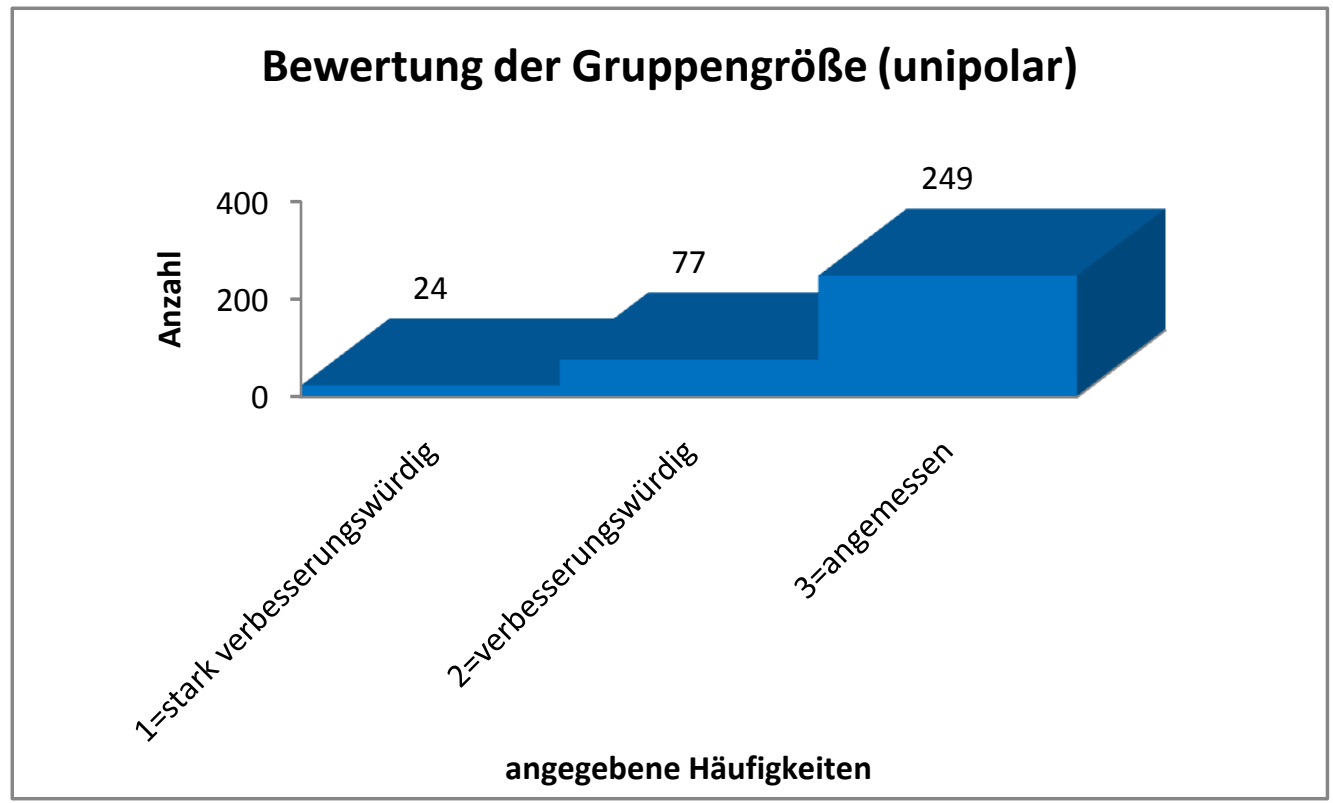

Abb.8: Bewertung der Gruppengröße auf unipolarer Skala (MW=2,64; SD=0,61; $\mathrm{N}=350$ )

Benotung des Entspannungskurses. Im Rahmen des Katamnesefragebogens wurden die Studienteilnehmer gebeten, eine Bewertung des Entspannungskurses auf einer Schulnotenskala von 1-6 vorzunehmen. Dazu waren auch alle verbliebenen 274 Patienten bereit. Die durchschnittliche Benotung des Entspannungstrainings ergab eine „gute“ 2 (MW=1,97; SD=0,99). Darüber hinaus hatten die Kursteilnehmer die Möglichkeit, den Inhalt des Grundlagenseminars (,viel zu schwierig“ bis „viel zu einfach“), den Vortragsstil (auf einer Notenskala von „sehr gut" bis „ungenügend") sowie die ausgegebenen Materialien (auch mittels Schulnoten) zu bewerten. Es zeigte sich eine schwache Korrelation der alltäglichen Übungs- 
häufigkeit nach drei Monaten mit der Variable „Benotung des Entspannungstrainings“ $(\mathbf{r}=+\mathbf{0 , 2 4 3} ; \mathrm{p}<0,01)$. Die positive Bewertung des Entspannungskurses hatte demnach einen positiven Einfluss auf den Alltagstransfer. Die folgende Abbildung veranschaulicht die Notenverteilung in Form eines Säulendiagramms.

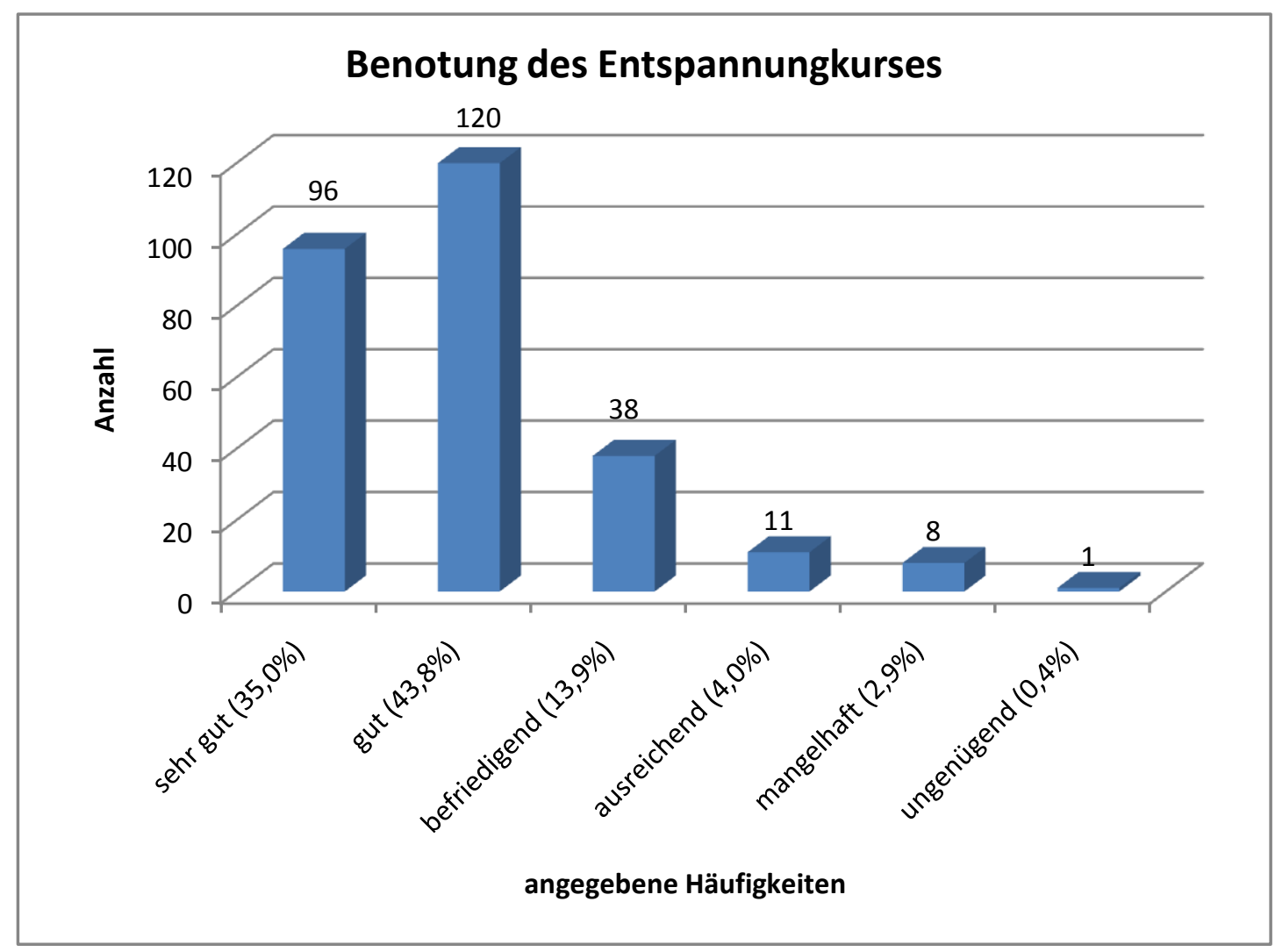

Abb.9: Benotung des Entspannungskurses ( $\mathrm{MW}=1,97 ; \mathrm{SD}=0,99 ; \mathrm{N}=274$ )

Durchschnittlich wurde der Inhalt des Grundlagenseminars mit ,gerade richtig“ eingeschätzt (MW=3,00; SD=0,28; N=274, s. Tab.16). Der Vortragsstil des Entspannungstherapeuten wurde durchschnittlich mit 1,84 ( $\mathrm{SD}=0,81 ; \mathrm{N}=274)$, die ausgeteilten Übungstexte mit 2,44 $(\mathrm{SD}=1,03 ; \mathrm{N}=246)$ bewertet.

Alter und Geschlecht. Das Alter lag im Mittel bei 49,14 \pm 8,48 Jahren. Der älteste Patient war 74 Jahre, der jüngste 19 Jahre alt. Zu Beginn der Studie waren 303 Patienten weiblichen Geschlechts (73,7\%), 108 Patienten waren Männer (26,3\%).

Es zeigte sich hinsichtlich des Alters eine schwache Korrelation $(\mathbf{r}=\mathbf{- 0 , 1 2 7} ; \mathrm{p}<0,05)$ - somit scheint es älteren Patienten etwas leichter zu fallen, die Entspannungsübungen erfolgreich in ihren Alltag zu integrieren. Das Geschlecht hatte dahingegen keinerlei Einfluss auf die Übungshäufigkeit zuhause. 
Die durchschnittliche Übungshäufigkeit der Patienten über und unter 40 Jahren unterschied sich nicht signifikant voneinander $(\mathrm{t}=0,584 ; \mathrm{n} . \mathrm{s}$.$) .$

Tab.16: Bewertung des Inhalts des Grundlagenseminars

\begin{tabular}{|l|l|}
\hline Benotung des Grundlagenseminars & Anzahl \\
\hline Viel zu schwierig & 0 \\
\hline Etwas zu schwierig & $3,3 \%$ (9 Pat.) \\
\hline Gerade richtig & $94,2 \%$ (258 Pat.) \\
\hline Etwas zu einfach & $1,8 \%$ (5 Pat.) \\
\hline Viel zu einfach & $0,7 \%$ (2 Pat.) \\
\hline
\end{tabular}

Aktuelles subjektives Wohlbefinden zum Zeitpunkt T3. Das aktuelle Wohlbefinden konnte auf einer Skala von 1 (,sehr unwohl“) bis 7 (,sehr wohl“) eingeschätzt werden und lag nach drei Monaten im Mittel bei 4,24 ( $\mathrm{SD}=1,48, \mathrm{~N}=268$; s. Abb.10).

Es fand sich allerdings nur eine schwach ausgeprägte Korrelation dieser Variablen mit der Übungshäufigkeit zu T3 (r=-0,165; p<0,01). Ein gutes persönliches Befinden scheint einen positiven Einfluss auf die Alltagstransfer zu haben.

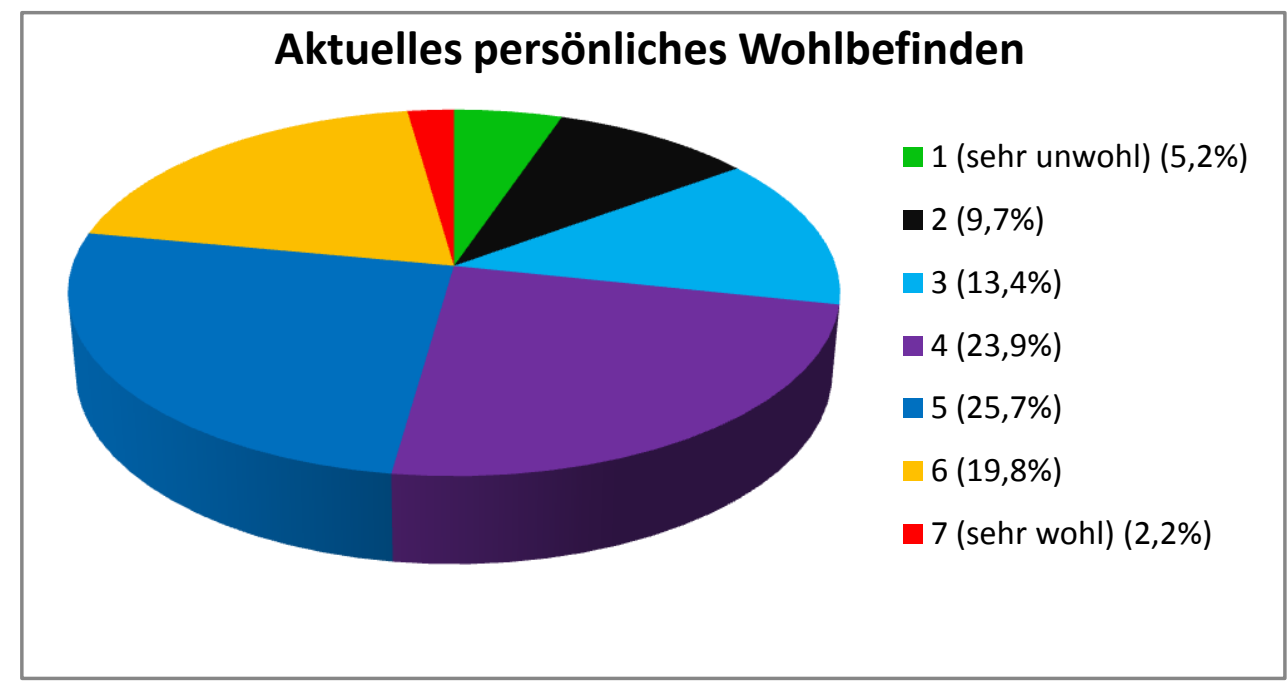

Abb.10: Bewertung des aktuellen persönlichen Wohlbefindens zu T3 
Lineare Regression. Um herauszufinden, durch welche als signifikant identifizierte Prädiktoren die Übungshäufigkeit im Alltag hauptsächlich erklärt werden kann, wurde den bivariaten Korrelationen nach Spearman-Rho eine lineare Regressionsanalyse angeschlossen. Die nachfolgende Tabelle veranschaulicht die Resultate.

Tab.17: Übersicht über die Ergebnisse der linearen Regressionsanalyse

\begin{tabular}{|l|c|c|l|}
\hline Mögliche Prädiktoren & Beta & $t$ & Sign. \\
\hline $\begin{array}{l}\text { Übungshäufigkeit während des Klinik- } \\
\text { aufenthalts außerhalb der Kursstunden }\end{array}$ & 0,506 & 9,937 & $p<0,01$ \\
\hline Verändertes Entspannungserleben zu T2 & 0,001 & 0,016 & n. s. \\
\hline Verändertes Entspannungserleben zu T3 & $-0,275$ & $-3,927$ & p<0,01 \\
\hline Benotung des Entspannungskurses & 0,077 & 1,391 & n. s. \\
\hline Alter der Patienten & $-0,061$ & $-1,221$ & n. s. \\
\hline Aktuelles subjektives Wohlbefinden zu T3 & 0,056 & 0,889 & n. s. \\
\hline $\mathbf{R}$ & $\mathbf{0 , 6 4 0}$ & $\mathbf{0 , 4 0 9}$ & \\
\hline $\mathbf{R}^{\mathbf{2}}$ & & & \\
\hline
\end{tabular}

Die multiple Korrelation beträgt $\mathrm{R}=0,640.40,9 \%$ der Varianzen im Kriterium können durch die Prädiktoren erklärt werden $\left(\mathrm{R}^{2}=0,409\right)$. Der signifikante Effekt der Variablen „Übungshäufigkeit während des Klinikaufenthalts außerhalb der Kursstunden“ (Beta=0,506; p<0,01) und „,verändertes Entspannungserleben zu T3“ (Beta=-0,275; p<0,01) blieb auch im Rahmen der linearen Regressionsanalyse signifikant. Der signifikante Effekt der übrigen Variablen ging im Rahmen der gemeinsamen Betrachtung zurück. 


\subsection{Zusammenhang zwischen der Übungshäufigkeit im Alltag und anspannungsbezogenen Symptomen bzw. Entspannungserleben}

\subsubsection{Symptome und Symptomentwicklung im Patientenkollektiv allgemein}

Die Studienteilnehmer wurden zu allen drei Zeitpunkten im Rahmen des Symptomfragebogens $\mathrm{zu}$ ihren aktuellen Beschwerden hinsichtlich körperlicher und psychischer Erschöpfung, Nervosität und innerer Anspannung, psychophysiologischer Dysregulation, Leistungs- und Verhaltensschwierigkeiten, Schmerzbelastung, und Problemen mit Selbstbestimmung und Selbstkontrolle befragt. Diese konnten auf einer Skala von 0 (,nie“) bis 3 (,stark“) eingeschätzt werden. Um die durchschnittliche Symptombelastung sowie ihre Entwicklung zu erfassen, wurden Mittelwerte der Skalen dieses Fragebogens gebildet und diese zu den unterschiedlichen Messzeitpunkten mittels t-Tests für verbundene Stichproben miteinander verglichen. Zudem wurden Effektstärken berechnet, wobei die stärksten Effekte innerhalb der ersten beiden Skalen der anspannungsbezogenen Beschwerden ermittelt werden konnten (s. Tab.18).

Die Beschwerden aller sechs Symptomskalen nahmen von T1 zu T2 signifikant ab ( $<<0,01)$. Die Symptombelastung nach drei Monaten blieb signifikant unter dem Ausgangsniveau von $\mathrm{T} 1(\mathrm{p}<0,01)$. 
Tab.18: Mittelwertvergleiche der Skalen des AT-SYM, Veränderungen zwischen T1, T2 und T3 und Effektstärken

\begin{tabular}{|c|c|c|c|c|c|}
\hline Skala & T1 & $\mathrm{T} 2$ & T3 & T1 vs. T2 & T1 vs. T3 \\
\hline $\begin{array}{l}\text { Körperliche und psychische } \\
\text { Erschöpfung }\end{array}$ & $\begin{array}{l}2,22 \\
(S D=0,53)\end{array}$ & $\begin{array}{l}1,27 \\
(S D=0,63)\end{array}$ & $\begin{array}{l}1,54 \\
(S D=0,68)\end{array}$ & $\begin{array}{l}t=27,87 \\
p<0,01 ; \\
E S=1,63\end{array}$ & $\begin{array}{l}t=15,55 \\
p<0,01 ; \\
E S=1,09\end{array}$ \\
\hline $\begin{array}{l}\text { Nervosität und innere } \\
\text { Anspannung }\end{array}$ & $\begin{array}{l}1,84 \\
(S D=0,61)\end{array}$ & $\begin{array}{l}1,04 \\
(S D=0.68)\end{array}$ & $\begin{array}{l}1,24 \\
(S D=0,72)\end{array}$ & $\begin{array}{l}t=21,68 ; \\
p<0,01 ; \\
E S=1,24\end{array}$ & $\begin{array}{l}t=12,66 ; \\
p<0,01 ; \\
E S=0,85\end{array}$ \\
\hline $\begin{array}{l}\text { Psychophysiologische } \\
\text { Dysregulation }\end{array}$ & $\begin{array}{l}1,21 \\
(S D=0,62)\end{array}$ & $\begin{array}{l}0,82 \\
(S D=0,59)\end{array}$ & $\begin{array}{l}0,92 \\
(S D=0,62)\end{array}$ & $\begin{array}{l}t=13,70 ; \\
p<0,01 ; \\
E S=0,64\end{array}$ & $\begin{array}{l}t=8,28 \\
p<0,01 \\
E S=0,42\end{array}$ \\
\hline $\begin{array}{l}\text { Leistungs- und } \\
\text { Verhaltensschwierigkeiten }\end{array}$ & $\begin{array}{l}1,58 \\
(S D=0,67)\end{array}$ & $\begin{array}{l}1,07 \\
(S D=0,73)\end{array}$ & $\begin{array}{l}1,14 \\
(S D=0,74)\end{array}$ & $\begin{array}{l}t=13,41 ; \\
p<0,01 \\
E S=0,71\end{array}$ & $\begin{array}{l}t=9,96 \\
p<0,01 \\
E S=0,58\end{array}$ \\
\hline Schmerzbelastung & $\begin{array}{l}1,31 \\
(S D=0,65)\end{array}$ & $\begin{array}{l}0,87 \\
(S D=0,63)\end{array}$ & $\begin{array}{l}1,06 \\
(S D=0,67)\end{array}$ & $\begin{array}{l}t=16,33 ; \\
p<0,01 ; \\
E S=0,68\end{array}$ & $\begin{array}{l}t=7,17 \\
p<0,01 ; \\
E S=0,35\end{array}$ \\
\hline $\begin{array}{l}\text { Selbstbestimmung und } \\
\text { Selbstkontrolle }\end{array}$ & $\begin{array}{l}1,57 \\
(S D=0,70)\end{array}$ & $\begin{array}{l}0,99 \\
(S D=0,73)\end{array}$ & $\begin{array}{l}1,09 \\
(S D=0,77)\end{array}$ & $\begin{array}{l}t=15,46 ; \\
p<0,01 ; \\
E S=0,80\end{array}$ & $\begin{array}{l}t=9,79 \\
p<0,01 \\
E S=0,60\end{array}$ \\
\hline
\end{tabular}

Korrelation der anspannungsbezogenen Symptome mit der Übungshäufigkeit zu T3. Als anspannungsbezogene Symptome können die ersten beiden Skalen „körperliche und psychische Erschöpfung“ und „Nervosität und innere Anspannung“ bezeichnet werden. Die Mittelwerte dieser Skalen zu T1, T2 und T3 wurden mit der Übungshäufigkeit im Alltag nach drei Monaten korreliert (bivariate Korrelation nach Spearman-Rho). Die folgende Tabelle fasst die Ergebnisse zusammen. 
Tab.19: Korrelation der Übungshäufigkeit im Alltag mit anspannungsbezogenen Symptomen zu den drei Messzeitpunkten T1, T2, T3

\begin{tabular}{|l|l|}
\hline Variablen & $\begin{array}{l}\text { Übungshäufigkeit im Alltag nach drei } \\
\text { Monaten }(N=274)\end{array}$ \\
\hline Körperliche und psychische Erschöpfung & T1: $r=-0,082 ; n$. s.; $N=411$ \\
T2: $r=+0,083 ; n . ~ s . ; N=350$ \\
\hline T3: $r=+0,199 ; p<0,01 ; N=269$ \\
$T 1: r=-0,035 ; n . s . ; N=411$ \\
$T 2: r=+0,128 ; p<0,05 ; N=350$ \\
$T 3: r=+0,198 ; p<0,01 ; N=266$ \\
\hline
\end{tabular}

Hochsignifikante Korrelationen wurden zum Zeitpunkt T3 erfasst $(\mathrm{p}<0,01)$ - es bestand allerdings nur ein schwacher Zusammenhang ( $r=+0,199$ bzw. $r=+0,198)$. Je weniger anspannungsbezogene Beschwerden im Alltag bestanden, desto wahrscheinlicher war ein erfolgreicher Alltagstransfer der Entspannungsübungen.

Korrelation der Symptomreduktion mit der Übungshäufigkeit zu T3. Um herauszufinden, ob ein Zusammenhang zwischen der Übungshäufigkeit im Alltag und der Entwicklung anspannungsbezogener Symptome besteht, wurden Differenzwerte der Skalenmittelwerte zwischen den Messzeitpunkten berechnet und diese wiederum mit der Übungshäufigkeit korreliert. Eine Übersicht der Ergebnisse liefert die folgende Tabelle.

Es konnte eine schwache Korrelation zwischen der Übungshäufigkeit im Alltag und der

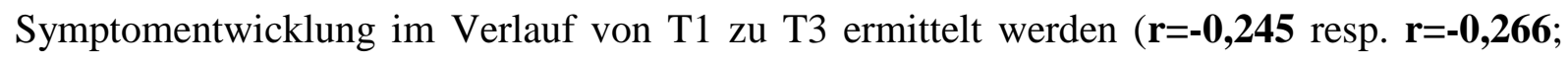
$\mathrm{p}<0,01)$. Je ausgeprägter die Symptomentwicklung, also die Symptomreduktion, zwischen Beginn der Reha und drei Monate nach Entlassung empfunden wurde, desto erfolgreicher war der Alltagstransfer. 
Tab.20: Korrelation der Symptomreduktion zwischen den Messzeitpunkten mit der Übungshäufigkeit im Alltag

\begin{tabular}{|l|l|}
\hline Variablen & $\begin{array}{l}\text { Übungshäufigkeit im Alltag nach drei } \\
\text { Monaten (N=274) }\end{array}$ \\
\hline $\begin{array}{l}\text { Reduktion körperlicher und psychischer Er- } \\
\text { schöpfung (T1-T2) }\end{array}$ & $r=-0,149 ; p<0,05(N=350)$ \\
\hline $\begin{array}{l}\text { Reduktion von Nervosität und innerer An- } \\
\text { spannung (T1-T2) }\end{array}$ & $r=-0,157 ; p<0,01(N=350)$ \\
\hline $\begin{array}{l}\text { Reduktion körperlicher und psychischer Er- } \\
\text { schöpfung (T2-T3) }\end{array}$ & $r=-0,139 ; p<0,05(N=269)$ \\
\hline $\begin{array}{l}\text { Reduktion von Nervosität und innerer An- } \\
\text { spannung (T2-T3) }\end{array}$ & $r=-0,065 ; n . s .(N=266)$ \\
\hline $\begin{array}{l}\text { Reduktion körperlicher und psychischer Er- } \\
\text { schöpfung (T1-T3) }\end{array}$ & $r=-0,245 ; p<0,01(N=269)$ \\
\hline spannung (T1-T3) & $r=-0,206 ; p<0,01(N=266)$ \\
\hline
\end{tabular}




\subsubsection{Symptome, Symptomentwicklung und verändertes Entspannungserleben im Vergleich „Übende“ vs. „Nicht-Übende“}

Um die Auswirkungen des regelmäßigen Trainings auf die (anspannungsbezogenen) Beschwerden gezielter zu erfassen, wurde das Patientenkollektiv in „Übende“ und „NichtÜbende“ eingeteilt: Studienteilnehmer mit einer Übungshäufigkeit von mindestens einmal pro Woche wurden in die Gruppe der „übenden Patienten“ aufgenommen (N=138) - Patienten mit weniger Trainingseinheiten zählten zu den „Nicht-Übenden“ ( $N=136)$.

Darüber hinaus wurden die Symptome, deren Entwicklung, sowie das veränderte Entspannungserleben in den extremen Quartilen erfasst. Zu den „häufig Übenden“ zählten jene Studienteilnehmer, die mindestens einmal pro Tag die Entspannungsübungen durchführten (N=43) - die „Nicht-Übenden“ waren diejenigen, die das Training seltener oder seit dem Einführungskurs gar nicht (mehr) anwandten (N=75).

Abschließend wurden die „häufig Übenden“ den „,regelmäßig Übenden“ (Trainingseinheiten von einmal bis mehrmals pro Woche, $\mathrm{N}=95$ ) und die „Nicht-Übenden“ den ,,selten Übenden“ (Übungshäufigkeit von einmal bis mehrmals pro Monat, $\mathrm{N}=61$ ) gegenübergestellt.

Mittelwerte zu T1, T2, T3 im Vergleich „Übende“ vs. „Nicht-Übende“. Die Skalenmittelwerte der „Übenden“ zu T1, T2 und T3 wurden denen der „Nicht-Übenden“ gegenübergestellt und mittels t-Tests für unabhängige Stichproben miteinander verglichen. Die folgende Tabelle liefert eine Übersicht der Symptombelastung zu den verschiedenen Messzeitpunkten (Tab.21). Die unterschiedliche Anzahl ergibt sich daraus, dass nicht alle Patienten Angaben zu ihren Beschwerden bei Ankunft, Abreise und nach drei Monaten machten.

Zu den Zeitpunkten T1 und T2 unterschieden sich die Symptome der „Übenden“ und „NichtÜbenden“ nicht signifikant voneinander - mit einer Ausnahme: bei Abreise gaben die „Übenden“ hochsignifikant weniger Beschwerden auf der Skala „Nervosität und innere Anspannung“ an als die „Nicht-Übenden“ $(\mathrm{t}=2,788 ; \mathrm{p}<0,01)$.

Nach drei Monaten bestanden hochsignifikante Unterschiede in der Symptombelastung der „Übenden“ vs. „Nicht-Übenden“ hinsichtlich „körperlicher und psychischer Erschöpfung“ $(\mathrm{t}=3,051 ; \mathrm{p}<0,01)$ und „Nervosität und innerer Anspannung“ $(\mathrm{t}=2,791 ; \mathrm{p}<0,01)$. Auf den übrigen Skalen konnten auch zu T3 keine signifikanten Unterschiede ermittelt werden. 
Tab.21: Mittelwertvergleiche der Skalen des AT-SYM zwischen „Übenden“ und „NichtÜbenden“ zu den verschiedenen Messzeitpunkten

\begin{tabular}{|c|c|c|c|}
\hline Skala & T1 & $\mathrm{T} 2$ & T3 \\
\hline \multirow{3}{*}{$\begin{array}{l}\text { Körperliche und } \\
\text { psychische } \\
\text { Erschöpfung }\end{array}$} & $\begin{array}{l}\text { Übende: } \mathbf{2 , 2 5} ; \\
S D=0,51 ; N=138\end{array}$ & $\begin{array}{l}\text { Übende: 1,18; } \\
\text { SD=0,61; N=138 }\end{array}$ & $\begin{array}{l}\text { Übende: 1,42; } \\
\text { SD=0,66; N=135 }\end{array}$ \\
\hline & $\begin{array}{l}\text { Nicht-Übende: 2,16; } \\
S D=0,53 ; N=136\end{array}$ & $\begin{array}{l}\text { Nicht-Übende: 1,30; } \\
S D=0,63 ; N=136\end{array}$ & $\begin{array}{l}\text { Nicht-Übende:1,66; } \\
\text { SD=0,68; N=134 }\end{array}$ \\
\hline & $\mathrm{t}=-1,328 ; \mathrm{n} . \mathrm{s}$. & $\mathrm{t}=1,636 ; \mathrm{n} . \mathrm{s}$. & $t=3,051 ; p<0,01$ \\
\hline \multirow{3}{*}{$\begin{array}{l}\text { Nervosität und innere } \\
\text { Anspannung }\end{array}$} & $\begin{array}{l}\text { Übende: 1,81; } \\
\text { SD=0,63; N=138 }\end{array}$ & $\begin{array}{l}\text { Übende: } \mathbf{0 , 8 9} ; \\
S D=0,63 ; N=138\end{array}$ & $\begin{array}{l}\text { Übende: 1,12; } \\
\text { SD=0,70; N=133 }\end{array}$ \\
\hline & $\begin{array}{l}\text { Nicht-Übende: 1,83; } \\
S D=0,64 ; N=136\end{array}$ & $\begin{array}{l}\text { Nicht-Übende: 1,12; } \\
\text { SD=0,70; N=136 }\end{array}$ & $\begin{array}{l}\text { Nicht-Übende: 1,36; } \\
S D=0,72 ; N=133\end{array}$ \\
\hline & $\mathrm{t}=0,199 ; \mathrm{n} . \mathrm{s}$. & $t=2,788 ; p<0,01$ & $t=2,791 ; p<0,01$ \\
\hline \multirow{3}{*}{$\begin{array}{l}\text { Psychophysiolog. } \\
\text { Dysregulation }\end{array}$} & $\begin{array}{l}\text { Übende: 1,22; } \\
\text { SD=0,66; N=138 }\end{array}$ & $\begin{array}{l}\text { Übende: } \mathbf{0 , 7 9 ;} \\
S D=0,62 ; N=138\end{array}$ & $\begin{array}{l}\text { Übende: } \mathbf{0 , 9 1 ;} \\
S D=0,63 ; N=133\end{array}$ \\
\hline & $\begin{array}{l}\text { Nicht-Übende: 1,15; } \\
\text { SD=0,59; N=136 }\end{array}$ & $\begin{array}{l}\text { Nicht-Übende: } \mathbf{0 , 8 3} ; \\
\text { SD=0,55; } N=136\end{array}$ & $\begin{array}{l}\text { Nicht-Übende: 0,92; } \\
\text { SD=0,61; N=133 }\end{array}$ \\
\hline & $t=-0,913 ; n . s$. & $\mathrm{t}=0,629 ; \mathrm{n} . \mathrm{s}$. & $\mathrm{t}=0,087 ;$ n. s. \\
\hline \multirow{3}{*}{$\begin{array}{l}\text { Leistungs- und } \\
\text { Verhaltens- } \\
\text { schwierigkeiten }\end{array}$} & $\begin{array}{l}\text { Übende: } 1,54 ; \\
S D=0,64 ; N=138\end{array}$ & $\begin{array}{l}\text { Übende: 0,98; } \\
\text { SD=0,70; N=138 }\end{array}$ & $\begin{array}{l}\text { Übende: } 1,09 ; \\
S D=0,71 ; N=133\end{array}$ \\
\hline & $\begin{array}{l}\text { Nicht-Übende: 1,57; } \\
\text { SD=0,72; N=136 }\end{array}$ & $\begin{array}{l}\text { Nicht-Übende: 1,08; } \\
\text { SD=0,73; N=134 }\end{array}$ & $\begin{array}{l}\text { Nicht-Übende: 1,18; } \\
S D=0,76 ; N=133\end{array}$ \\
\hline & $\mathrm{t}=0,397 ; \mathrm{n} . \mathrm{s}$. & $\mathrm{t}=1,142 ; \mathrm{n} . \mathrm{s}$. & $\mathrm{t}=0,978 ; n . s$. \\
\hline \multirow{3}{*}{ Schmerzbelastung } & $\begin{array}{l}\text { Übende: 1,34; } \\
S D=0,66 ; N=138\end{array}$ & $\begin{array}{l}\text { Übende: } \mathbf{0 , 8 4} ; \\
S D=0,65 ; N=138\end{array}$ & $\begin{array}{l}\text { Übende: } \mathbf{1 , 0 3} ; \\
S D=0,66 ; N=133\end{array}$ \\
\hline & $\begin{array}{l}\text { Nicht-Übende: 1,26; } \\
\text { SD=0,65; N=135 }\end{array}$ & $\begin{array}{l}\text { Nicht-Übende: } \mathbf{0 , 8 8} \\
S D=0,61 ; N=136\end{array}$ & $\begin{array}{l}\text { Nicht-Übende: 1,09; } \\
\text { SD=0,68; N=133 }\end{array}$ \\
\hline & $\mathrm{t}=-0,986 ; n . s$. & $\mathrm{t}=0,502 ; \mathrm{n} . \mathrm{s}$. & $\mathrm{t}=0,729 ; \mathrm{n} . \mathrm{s}$. \\
\hline
\end{tabular}




\begin{tabular}{|c|c|c|c|}
\hline Skala & $\mathrm{T} 1$ & $\mathrm{~T} 2$ & T3 \\
\hline \multirow{3}{*}{$\begin{array}{l}\text { Selbstbestimmung und } \\
\text { Selbstkontrolle }\end{array}$} & $\begin{array}{l}\text { Übende: 1,50; } \\
\text { SD=0,76; } N=138\end{array}$ & $\begin{array}{l}\text { Übende: } \mathbf{0 , 9 0 ;} \\
S D=0,72 ; N=137\end{array}$ & $\begin{array}{l}\text { Übende: 1,02; } \\
S D=0,76 ; N=131\end{array}$ \\
\hline & $\begin{array}{l}\text { Nicht-Übende: 1,56; } \\
\text { SD=0,69; N=136 }\end{array}$ & $\begin{array}{l}\text { Nicht-Übende: 1,02; } \\
S D=0,73 ; N=134\end{array}$ & $\begin{array}{l}\text { Nicht-Übende: 1,16; } \\
\text { SD=0,78; N=131 }\end{array}$ \\
\hline & $\mathrm{t}=0,592 ; \mathrm{n} . \mathrm{s}$ & $\mathrm{t}=1,403 ;$ n. $s$. & t=1,449; n. s. \\
\hline
\end{tabular}

Vergleich der „Übenden“ vs. „Nicht-Übenden“ bezogen auf die Übungshäufigkeit während des Klinikaufenthalts. Die vorausgegangene Tabelle basiert auf den Angaben der Studienteilnehmer nach drei Monaten (N=274). Hinsichtlich Übungshäufigkeit in der Klinik stehen 243 „Übenden“ 107 „Nicht-Übende“ gegenüber $\left(\mathrm{N}_{\text {gesamt }}=350\right)$. Weder der Vergleich der bestehenden anspannungsbezogenen Symptome am Ende der Rehabilitation noch die Symptomentwicklung (Berechnung von Differenzwerten) ergab signifikante Ergebnisse zwischen den Gruppen (s. Tab.22). Lediglich das veränderte Entspannungserleben am Ende des Klinikaufenthalts unterschied sich hochsignifikant $(t=-2,730 ; p<0,01)$.

Tab.22: Mittelwertvergleich (T1, T2), Vergleich der Differenzwerte (T1-T2) und des veränderten Entspannungserlebens zu T2 ( $\left.\mathrm{N}_{\text {gesamt }}=350\right)$ zwischen „Übenden“ und „Nicht-Übenden“

\begin{tabular}{|c|c|c|c|}
\hline Skala & $\mathrm{T} 1$ & $\mathrm{~T} 2$ & Sign. (2-seitig) \\
\hline \multirow{2}{*}{$\begin{array}{l}\text { Körperliche und psychi- } \\
\text { sche Erschöpfung }\end{array}$} & $\begin{array}{l}\text { Übende: 2,25; } \\
S D=0,51 ; N=243\end{array}$ & $\begin{array}{l}\text { Übende: 1,28; } \\
S D=0,62 ; N=243\end{array}$ & \multirow{2}{*}{$\begin{array}{l}t(T 1)=-1,297 ; n . s . \\
t(T 2)=-0,353 ; n . s .\end{array}$} \\
\hline & $\begin{array}{l}\text { Nicht-Übende: 2,17; } \\
\text { SD=0,57; N=107 }\end{array}$ & $\begin{array}{l}\text { Nicht-Übende: 1,25; } \\
\text { SD=0,64; N=107 }\end{array}$ & \\
\hline \multirow{2}{*}{$\begin{array}{l}\text { Nervosität und innere } \\
\text { Anspannung }\end{array}$} & $\begin{array}{l}\text { Übende: 1,84; } \\
S D=0,60 ; N=243\end{array}$ & $\begin{array}{l}\text { Übende: 1,02; } \\
\text { SD=0,67; N=243 }\end{array}$ & \multirow{2}{*}{$\begin{array}{l}t(T 1)=-0,165 ; n . s . \\
t(T 2)=0,597 ; n . s .\end{array}$} \\
\hline & $\begin{array}{l}\text { Nicht-Übende: 1,83; } \\
\text { SD=0,64; N=107 }\end{array}$ & $\begin{array}{l}\text { Nicht-Übende: 1,07; } \\
\text { SD=0,70; N=107 }\end{array}$ & \\
\hline
\end{tabular}




\begin{tabular}{|c|c|c|}
\hline \multirow{2}{*}{$\begin{array}{l}\text { Reduktion körperlicher } \\
\text { und psychischer Er- } \\
\text { schöpfung (T1-T2) }\end{array}$} & $\begin{array}{l}\text { Übende: } \mathbf{0 , 9 7} ; \\
S D=0,65 ; N=243\end{array}$ & \multirow{2}{*}{$\mathrm{t}=-0,720 ; \mathrm{n} . \mathrm{s}}$. \\
\hline & $\begin{array}{l}\text { Nicht-Übende: } \mathbf{0 , 9 2 ;} \\
S D=0,62 ; N=107\end{array}$ & \\
\hline \multirow{2}{*}{$\begin{array}{l}\text { Reduktion von Nervosität } \\
\text { und innerer Anspannung } \\
\text { (T1-T2) }\end{array}$} & $\begin{array}{l}\text { Übende: } \mathbf{0 , 8 2} \\
S D=0,70 ; N=243\end{array}$ & \multirow{2}{*}{$\mathrm{t}=-0,732 ; \mathrm{n} . \mathrm{s}$} \\
\hline & $\begin{array}{l}\text { Nicht-Übende: } \mathbf{0 , 7 6} ; \\
\text { SD=0,68; N=107 }\end{array}$ & \\
\hline \multirow{2}{*}{$\begin{array}{l}\text { Verändertes Entspan- } \\
\text { nungserleben (T2) }\end{array}$} & $\begin{array}{l}\text { Übende: 5,14; } \\
S D=0,96 ; N=242\end{array}$ & \multirow{2}{*}{$t=-2,730 ; p<0,01$} \\
\hline & $\begin{array}{l}\text { Nicht-Übende: 4,83; } \\
\text { SD=1,01; N=107 }\end{array}$ & \\
\hline
\end{tabular}

\section{Vergleich „Übende“ vs. „Nicht-Übende“ hinsichtlich Symptomentwicklung (T1-T3, T2-}

T3) und veränderten Entspannungserlebens zu T3. Im nächsten Schritt wurde die Entwicklung der anspannungsbezogenen Beschwerden, also die Symptomreduktion, sowohl von Beginn des Klinikaufenthalts bis zur postalischen Befragung nach drei Monaten (T1-T3) als auch vom Tag der Abreise bis zur postalischen Befragung (T2-T3) untersucht (s. Tab.23).

Die Ergebnisse können wie folgt zusammengefasst werden:

Vom Tag der Abreise bis zur Befragung nach drei Monaten (T2-T3) unterschieden sich die „Übenden“ nicht signifikant von den „Nicht-Übenden“ hinsichtlich einer möglichen Symptomreduktion. Betrachtet man die Entwicklung der anspannungsbezogenen Beschwerden vom Beginn des Rehabilitationsaufenthalts bis drei Monate nach Entlassung (T1-T3) besteht jedoch ein (hoch)signifikanter Unterschied zwischen den beiden Gruppen ( $t=-3,917 ; \mathrm{p}<0,01$ resp. $\mathrm{t}=-2,153 ; \mathrm{p}<0,05)$. Das veränderte Entspannungserleben und Wohlbefinden der „Übenden“ nach drei Monaten unterschied sich hochsignifikant von jenem der „Nicht-Übenden“ $(\mathrm{t}=-5,124 ; \mathrm{p}<0,01)$. 
Tab.23: Vergleich „Übende“ vs. „Nicht-Übende“ hinsichtlich Symptomreduktion (T1-T3, T2T3) und veränderten Entspannungserlebens zu T3

\begin{tabular}{|c|c|c|c|}
\hline Skala & & & Sign. (2-seitig) \\
\hline $\begin{array}{l}\text { Reduktion körperlicher } \\
\text { und psychischer Er- } \\
\text { schöpfung (T1-T3) }\end{array}$ & $\begin{array}{l}\text { Übende: } \mathbf{0 , 8 3} ; \\
S D=0,68 ; N=135\end{array}$ & $\begin{array}{l}\text { Nicht-Übende: } \mathbf{0 , 5 0} ; \\
\text { SD=0,69; N=134 }\end{array}$ & $t=-3,917 ; p<0,01$ \\
\hline $\begin{array}{l}\text { Reduktion von Nervosität } \\
\text { und innerer Anspannung } \\
\text { (T1-T3) }\end{array}$ & $\begin{array}{l}\text { Übende: } \mathbf{0 , 6 7} ; \\
\text { SD=0,73; N=133 }\end{array}$ & $\begin{array}{l}\text { Nicht-Übende: } \mathbf{0 , 4 8} ; \\
\text { SD=0,74; } N=133\end{array}$ & $t=-2,153 ; p<0,05$ \\
\hline $\begin{array}{l}\text { Reduktion körperlicher } \\
\text { und psychischer Er- } \\
\text { schöpfung (T2-T3) }\end{array}$ & $\begin{array}{l}\text { Übende: } \mathbf{- 0 , 2 4 ;} \\
\text { SD=0,62; N=135 }\end{array}$ & $\begin{array}{l}\text { Nicht-Übende: -0,36; } \\
\text { SD=0,59; N=134 }\end{array}$ & $\mathrm{t}=-1,601 ; \mathrm{n} . \mathrm{s}$ \\
\hline $\begin{array}{l}\text { Reduktion von Nervosität } \\
\text { und innerer Anspannung } \\
\text { (T2-T3) }\end{array}$ & $\begin{array}{l}\text { Übende: }-\mathbf{0 , 2 4} ; \\
S D=0,59 ; N=133\end{array}$ & $\begin{array}{l}\text { Nicht-Übende: -0,23; } \\
\text { SD=0,54; N=133 }\end{array}$ & $\mathrm{t}=0,147 ; \mathrm{n} . \mathrm{s}$. \\
\hline $\begin{array}{l}\text { Verändertes Entspan- } \\
\text { nungserleben (T3) }\end{array}$ & $\begin{array}{l}\text { Übende: } \mathbf{5 , 1 1} ; \\
S D=0,94 ; N=133\end{array}$ & $\begin{array}{l}\text { Nicht-Übende: 4,45; } \\
\text { SD=1,15; N=129 }\end{array}$ & $t=-5,124 ; p<0,01$ \\
\hline
\end{tabular}

Extreme Quartile. Im Folgenden wurden die anspannungsbezogenen Symptome zu T3, deren Entwicklung im Verlauf T2-T3 und T1-T3 sowie das veränderte Entspannungserleben nach drei Monaten in den extremen Quartilen erfasst.

Dabei standen 43 Patienten, die mehrmals pro Tag übten, 75 Rehabilitanden gegenüber, die PR gar nicht mehr praktizierten. Das Alter der ,häufig Übenden“ lag im Mittel bei 51 Jahren ( $\mathrm{SD}=7,12) .74,4 \%$ (32 Patienten) waren weiblichen Geschlechts, 25,6\% (11 Patienten) waren Männer. Die Gruppe der „Nicht-Übenden“ umfasste 77,3\% Frauen (58 Patienten) und 22,7\% Männer (17 Patienten) mit einem durchschnittlichen Alter von 48,39 Jahren (SD=8,21). Es wurden nicht alle Fragen von sämtlichen Studienteilnehmern dieser Gruppen beantwortet, sodass eine davon abweichende Anzahl je nach Variable vorliegen kann. Die folgende Tabelle liefert eine Übersicht der Ergebnisse (Tab.24). 
Die „häufig Übenden“ gaben zu T3 hochsignifikant weniger anspannungsbezogene Beschwerden an $(\mathrm{t}=-2,889$ resp. $\mathrm{t}=-2,741 ; \mathrm{p}<0,01)$, konnten im Verlauf von $\mathrm{T} 1 \mathrm{zu} \mathrm{T} 3$ eine stärkere Symptomreduktion erreichen $(\mathrm{t}=3,728$ resp. $\mathrm{t}=3,121 ; \mathrm{p}<0,01)$ und unterschieden sich hinsichtlich eines veränderten Entspannungserlebens und Wohlbefindens nach drei Monaten $(\mathrm{t}=5,211 ; \mathrm{p}<0,01)$ von jenen Patienten, die selten übten oder die Übungen gänzlich eingestellt hatten.

Tab.24: Vergleich der extremen Quartile hinsichtlich anspannungsbezogener Symptome zu T3, deren Entwicklung (T1-T3, T2-T3) und veränderten Entspannungserlebens zu T3

\begin{tabular}{|c|c|c|c|}
\hline Skala & & & Sign. (2-seitig) \\
\hline $\begin{array}{l}\text { Körperliche und psychi- } \\
\text { sche Erschöpfung (T3) }\end{array}$ & $\begin{array}{l}\text { Häufig Übende: 1,33; } \\
S D=0,70 ; N=42\end{array}$ & $\begin{array}{l}\text { Nicht-Übende: 1,72; } \\
\text { SD=0,71; } N=74\end{array}$ & $t=-2,889 ; p<0,01$ \\
\hline $\begin{array}{l}\text { Nervosität und innere } \\
\text { Anspannung (T3) }\end{array}$ & $\begin{array}{l}\text { Häufig Übende: 1,08; } \\
S D=0,69 ; N=42\end{array}$ & $\begin{array}{l}\text { Nicht-Übende: 1,45; } \\
\text { SD=0,70; } N=73\end{array}$ & $\mathrm{t}=-2,741 ; \mathrm{p}<0,01$ \\
\hline $\begin{array}{l}\text { Reduktion körperlicher } \\
\text { und psychischer } \\
\text { Erschöpfung (T1-T3) }\end{array}$ & $\begin{array}{l}\text { Häufig Übende: } 1,00 ; \\
S D=0,73 ; N=42\end{array}$ & $\begin{array}{l}\text { Nicht-Übende: } \mathbf{0 , 4 7} \\
\text { SD=0,74; } N=74\end{array}$ & $t=3,728 ; p<0,01$ \\
\hline $\begin{array}{l}\text { Reduktion von Nervosität } \\
\text { und innerer Anspannung } \\
\text { (T1-T3) }\end{array}$ & $\begin{array}{l}\text { Häufig Übende: } \mathbf{0 , 8 5} ; \\
S D=0,86 ; N=42\end{array}$ & $\begin{array}{l}\text { Nicht-Übende: } \mathbf{0 , 3 7} ; \\
S D=0,77 ; N=73\end{array}$ & $t=3,121 ; p<0,01$ \\
\hline $\begin{array}{l}\text { Reduktion körperlicher } \\
\text { und psychischer } \\
\text { Erschöpfung (T2-T3) }\end{array}$ & $\begin{array}{l}\text { Häufig Übende: } \mathbf{0 , 2 0} ; \\
S D=0,80 ; N=42\end{array}$ & $\begin{array}{l}\text { Nicht-Übende: } \mathbf{0 , 4 1} ; \\
S D=0,62 ; N=74\end{array}$ & $t=1,607 ;$ n. s. \\
\hline $\begin{array}{l}\text { Reduktion von Nervosität } \\
\text { und innerer Anspannung } \\
\text { (T2-T3) }\end{array}$ & $\begin{array}{l}\text { Häufig Übende: -0,21; } \\
\text { SD=0,78; } N=42\end{array}$ & $\begin{array}{l}\text { Nicht-Übende: } \mathbf{0 , 3 1} ; \\
S D=0,51 ; N=73\end{array}$ & $\mathrm{t}=0,783 ; \mathrm{n} . \mathrm{s}$ \\
\hline $\begin{array}{l}\text { Verändertes Entspan- } \\
\text { nungserleben (T3) }\end{array}$ & $\begin{array}{l}\text { Häufig Übende: 5,31; } \\
S D=0,82 ; N=41\end{array}$ & $\begin{array}{l}\text { Nicht-Übende: 4,22; } \\
\text { SD=1,18; N=69 }\end{array}$ & $t=5,211 ; p<0,01$ \\
\hline
\end{tabular}


„Häufig Übende“6 vs. „regelmäßig Übende“ und „Nicht-Übende“ vs. „selten Übende“. Abschließend wurden die „häufig Übenden“ $(\mathrm{N}=43)$, die mindestens einmal täglich übten, den „,regelmäßig Übenden“ ( $\mathrm{N}=95)$, die Entspannungsübungen mindestens einmal bis mehrmals pro Woche durchführten, hinsichtlich anspannungsbezogener Symptome, deren Entwicklung und des veränderten Entspannungserlebens nach drei Monaten gegenübergestellt, ebenso die „Nicht-Übenden“ ( $\mathrm{N}=75)$ den „selten Übenden“ $(\mathrm{N}=61)$. Unter den „Nicht-Übenden“ wurden - wie oben definiert - jene Patienten zusammengefasst, die seit dem Entspannungskurs seltener oder gar nicht (mehr) PR-Übungen praktizierten, wohingegen die „selten Übenden“" wenigstens einmal bis mehrmals im Monat das Entspannungstraining in ihren Alltag integrierten. Die folgende Tabelle liefert eine Übersicht der Ergebnisse (Tab.25).

Zwischen den häufig und regelmäßig Übenden bestanden keine signifikanten Unterschiede hinsichtlich Symptombelastung nach drei Monaten, Symptomentwicklung von T2 zu T3 und verändertem Entspannungserleben zu T3. Lediglich im Vergleich der Symptomentwicklung von Beginn der Reha bis zur Katamnese (T1-T3) erbrachte der t-Test für unverbundene Stichproben ein signifikantes Ergebnis: Die Entwicklung der Beschwerden auf der Skala „,körperliche und psychische Erschöpfung“ der häufig Übenden unterschied sich auf dem 5\%-Niveau signifikant von der der regelmäßig Übenden $(t=2,029 ; p<0,05)$. Auf der Skala „Nervosität und innere Anspannung“" war dieser Unterschied nicht abbildbar ( $\mathrm{t}=1,750$; n. s.).

Die Gegenüberstellung der „Nicht-Übenden“ ( $N=75)$ mit den „selten Übenden“ ( $N=61)$ mittels t-Tests für unverbundene Stichproben (s. Tab.26) erbrachte lediglich ein signifikantes Ergebnis: Das veränderte Entspannungserleben und Wohlbefinden der „,selten Übenden“ nach drei Monaten unterschied sich auf dem 5\%-Niveau signifikant von dem der „Nicht-Übenden“ $(\mathrm{t}=-2,456 ; \mathrm{p}<0,05)$. Die Patienten, die wenigstens ein- bis mehrmals im Monat übten, verspürten nach drei Monaten eine stärkere Verbesserung ihres Entspannungserlebens und Wohlbefindens als jene, die das Training gar nicht (mehr) anwandten. Hinsichtlich Symptombelastung und Symptomentwicklung unterschieden sich die beiden Gruppen nicht signifikant voneinander. 


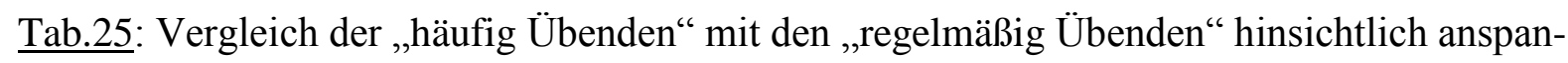
nungsbezogener Symptome zu T3, deren Entwicklung (T1-T3, T2-T3) und veränderten Entspannungserlebens zu T3

\begin{tabular}{|c|c|c|c|}
\hline Skala & & & Sign. (2-seitig) \\
\hline $\begin{array}{l}\text { Körperliche und psychi- } \\
\text { sche Erschöpfung (T3) }\end{array}$ & $\begin{array}{l}\text { Häufig Übende: 1,33; } \\
S D=0,70 ; N=42\end{array}$ & $\begin{array}{l}\text { Regelmäßig Übende:1,45; } \\
\text { SD=0,63; N=93 }\end{array}$ & $t=-1,020 ; n . s$. \\
\hline $\begin{array}{l}\text { Nervosität und innere } \\
\text { Anspannung (T3) }\end{array}$ & $\begin{array}{l}\text { Häufig Übende: 1,08; } \\
S D=0,69 ; N=42\end{array}$ & $\begin{array}{l}\text { Regelmäßig Übende: 1,14; } \\
\mathrm{SD}=0,71 ; \mathrm{N}=91\end{array}$ & $\mathrm{t}=-0,494 ; \mathrm{n} . \mathrm{s}$. \\
\hline $\begin{array}{l}\text { Reduktion körperlicher } \\
\text { und psychischer } \\
\text { Erschöpfung (T1-T3) }\end{array}$ & $\begin{array}{l}\text { Häufig Übende: 1,00; } \\
S D=0,73 ; N=42\end{array}$ & $\begin{array}{l}\text { Regelmäßig Übende: } \mathbf{0 , 7 5} \\
\mathrm{SD}=0,65 ; \mathrm{N}=93\end{array}$ & $\begin{array}{l}t=2,029 \\
p<0,05\end{array}$ \\
\hline $\begin{array}{l}\text { Reduktion von Nervosität } \\
\text { und innerer Anspannung } \\
\text { (T1-T3) }\end{array}$ & $\begin{array}{l}\text { Häufig Übende: } \mathbf{0 , 8 5} \text {; } \\
S D=0,86 ; N=42\end{array}$ & $\begin{array}{l}\text { Regelmäßig Übende: 0,59; } \\
\text { SD 0,65; N=91 }\end{array}$ & $\mathrm{t}=1,750 ;$ n. s. \\
\hline $\begin{array}{l}\text { Reduktion körperlicher } \\
\text { und psychischer } \\
\text { Erschöpfung (T2-T3) }\end{array}$ & $\begin{array}{l}\text { Häufig Übende: } \mathbf{- 0 , 2 0 ;} \\
S D=0,80 ; N=42\end{array}$ & $\begin{array}{l}\text { Regelmäßig Übende: -0,27; } \\
S D=0,53 ; N=93\end{array}$ & $\mathrm{t}=0,578 ; \mathrm{n} . \mathrm{s}$. \\
\hline $\begin{array}{l}\text { Reduktion von Nervosität } \\
\text { und innerer Anspannung } \\
\text { (T2-T3) }\end{array}$ & $\begin{array}{l}\text { Häufig Übende: -0,21; } \\
S D=0,78 ; N=42\end{array}$ & $\begin{array}{l}\text { Regelmäßig Übende: } \mathbf{- 0 , 2 6} ; \\
\mathrm{SD}=0,48 ; \mathrm{N}=91\end{array}$ & $\mathrm{t}=0,515 ; \mathrm{n} . \mathrm{s}$ \\
\hline $\begin{array}{l}\text { Verändertes Entspan- } \\
\text { nungserleben (T3) }\end{array}$ & $\begin{array}{l}\text { Häufig Übende: 5,31; } \\
S D=0,82 ; N=41\end{array}$ & $\begin{array}{l}\text { Regelmäßig Übende: 5,03; } \\
\mathrm{SD}=0,98 ; \mathrm{N}=92\end{array}$ & $\mathrm{t}=1,608 ;$ n. s. \\
\hline
\end{tabular}


Tab.26: Vergleich der „Nicht-Übenden“ mit den „,selten Übenden“ hinsichtlich anspannungsbezogener Symptome zu T3, deren Entwicklung (T1-T3, T2-T3) und veränderten Entspannungserlebens zu T3

\begin{tabular}{|c|c|c|c|}
\hline Skala & & & Sign. (2-seitig) \\
\hline $\begin{array}{l}\text { Körperliche und psychi- } \\
\text { sche Erschöpfung (T3) }\end{array}$ & $\begin{array}{l}\text { Nicht-Übende: 1,72; } \\
\text { SD=0,71; N=74 }\end{array}$ & $\begin{array}{l}\text { Selten Übende: 1,59; } \\
S D=0,65 ; N=60\end{array}$ & $\mathrm{t}=1,112 ; \mathrm{n} . \mathrm{s}$ \\
\hline $\begin{array}{l}\text { Nervosität und innere } \\
\text { Anspannung (T3) }\end{array}$ & $\begin{array}{l}\text { Nicht-Übende: } \mathbf{1 , 4 5} ; \\
\text { SD=0,70; N=73 }\end{array}$ & $\begin{array}{l}\text { SeltenÜbende: 1,26; } \\
S D=0,73 ; N=60\end{array}$ & $\mathrm{t}=1,478 ; \mathrm{n} . \mathrm{s}$. \\
\hline $\begin{array}{l}\text { Reduktion körperlicher } \\
\text { und psychischer } \\
\text { Erschöpfung (T1-T3) }\end{array}$ & $\begin{array}{l}\text { Nicht-Übende: } \mathbf{0 , 4 7} ; \\
S D=0,74 ; N=74\end{array}$ & $\begin{array}{l}\text { Selten Übende: } \mathbf{0 , 5 4} ; \\
S D=0,62 ; N=60\end{array}$ & $t=-0,504 ; n . s$. \\
\hline $\begin{array}{l}\text { Reduktion von Nervosität } \\
\text { und innerer Anspannung } \\
\text { (T1-T3) }\end{array}$ & $\begin{array}{l}\text { Nicht-Übende: } \mathbf{0 , 3 7} ; \\
S D=0,77 ; N=73\end{array}$ & $\begin{array}{l}\text { Selten Übende: } \mathbf{0 , 6 1} ; \\
S D=0,70 ; N=60\end{array}$ & $t=1,923 ; n . s$. \\
\hline $\begin{array}{l}\text { Reduktion körperlicher } \\
\text { und psychischer } \\
\text { Erschöpfung (T2-T3) }\end{array}$ & $\begin{array}{l}\text { Nicht-Übende: }-\mathbf{0 , 4 1} ; \\
S D=0,62 ; N=74\end{array}$ & $\begin{array}{l}\text { Selten Übende: }-\mathbf{0 , 3 0} \text {; } \\
S D=0,54 ; N=60\end{array}$ & $\mathrm{t}=-1,096 ; \mathrm{n} . \mathrm{s}$ \\
\hline $\begin{array}{l}\text { Reduktion von Nervosität } \\
\text { und innerer Anspannung } \\
\text { (T2-T3) }\end{array}$ & $\begin{array}{l}\text { Nicht-Übende: -0,31; } \\
S D=0,51 ; N=73\end{array}$ & $\begin{array}{l}\text { SeltenÜbende: }-\mathbf{0 , 1 4} ; \\
S D=0,58 ; N=60\end{array}$ & $\mathrm{t}=-1,809 ; \mathrm{n} . \mathrm{s}$ \\
\hline $\begin{array}{l}\text { Verändertes Entspan- } \\
\text { nungserleben (T3) }\end{array}$ & $\begin{array}{l}\text { Nicht-Übende: 4,22; } \\
\text { SD=1,18; N=69 }\end{array}$ & $\begin{array}{l}\text { Selten Übende: } \mathbf{4 , 7 1 ;} \\
S D=1,08 ; N=60\end{array}$ & $t=-2,456 ; p<0,05$ \\
\hline
\end{tabular}




\subsection{Zusammenfassung der Ergebnisse}

1. 69,4\% der Patienten konnten die Entspannungsübungen erfolgreich bereits während des Klinikaufenthalts umsetzen. Durchschnittlich übten sie einmal pro Woche außerhalb der Kursstunden. Nach konservativer Schätzung übte ein signifikant höherer Anteil der Studienteilnehmer von 2010 auch außerhalb der Kursstunden $(\mathrm{p}<0,01)$. Dem veränderten Entspannungserleben nach dem Kurs (T2) konnte ein - allerdings geringer - positiver Einfluss auf den erfolgreichen Kliniktransfer zugeschrieben werden $(r=-0,184 ; p<0,01)$.

2. 50,4\% der Studienteilnehmer konnten die Übungen der Progressiven Relaxation auch drei Monate nach Entlassung in ihren persönlichen Alltag integrieren. Im Durchschnitt übten sie mehrmals pro Monat. Die „Erfolgsquote“ von 2010 unterschied sich nicht von derjenigen der Pilotstudie aus dem Jahre 2006 (weder nach optimistischer noch nach konservativer Schätzung).

3. Die Übungshäufigkeit während des Klinikaufenthalts $(r=+0,558 ; p<0,01)$ und das veränderte Entspannungserleben und Wohlbefinden zu T3 $(r=-0,356 ; p<0,01)$ waren am stärksten mit einem erfolgreichen Alltagstransfer korreliert. Desweiteren fanden sich schwach ausgeprägte Korrelationen zwischen der Übungshäufigkeit im Alltag und dem veränderten Entspannungserleben bei Abreise $(r=-0,240 ; \mathrm{p}<0,01)$, der Benotung des Entspannungskurses $(\mathrm{r}=+0,243 ; \mathrm{p}<0,01)$ sowie dem aktuellen subjektiven Wohlbefinden zu T3 (r=-0,165; p<0,01). Das Alter der Patienten korrelierte nur schwach mit dem Alltagstransfer $(\mathrm{r}=-0,127 ; \mathrm{p}<0,05)$. Demnach war der erfolgreiche Alltagstransfer bei jenen Patienten wahrscheinlicher, die erfolgreich bereits während des Klinikaufenthalts übten, eine starke Veränderung ihres Entspannungserlebens und Wohlbefindens (zu T2 und vor allem zu T3) erfuhren, den Entspannungskurs positiv bewerteten und eine geringe Symptombelastung im Alltag aufwiesen. Zudem schien es älteren Patienten etwas leichter zu fallen, die Entspannungsübungen erfolgreich in ihren Alltag zu integrieren. Weder die Bewertung der Gruppengröße noch das Geschlecht hatten einen Einfluss auf die Übungshäufigkeit im Alltag.

4. Bei der gemeinsamen Betrachtung im Rahmen der linearen Regressionsanalyse blieb der Effekt der „Übungshäufigkeit während des Klinikaufenthalts außerhalb der Kursstunden“ (Beta=0,506; $p<0,01)$ und des ,veränderten Entspannungserlebens zu T3“ (Beta=-0,275; 
$\mathrm{p}<0,01)$ signifikant. Die multiple Korrelation betrug $\mathrm{R}=0,640$, d. h. 40,9\% der Varianzen im Kriterium können durch die Prädiktoren erklärt werden $\left(\mathrm{R}^{2}=0,409\right)$.

5. Das Entspannungserleben und Wohlbefinden aller Studienteilnehmer zeigte bei Abreise (T2) eine positive Veränderung (Mittelwert der 12 Items zu T2=5,044; SD=0,98; N=349), die auch nach drei Monaten in abgeschwächter Form noch nachweisbar war (Mittelwert der 12 Items zu T3=4,79; SD=1,1; N=262).

6. Die Beschwerden aller sechs Symptomskalen nahmen von $\mathrm{T} 1 \mathrm{zu}$ T2 signifikant ab $(\mathrm{p}<0,01)$. Die Symptombelastung nach drei Monaten blieb signifikant unter dem Ausgangsniveau von $\mathbf{T 1}(\mathbf{p}<\mathbf{0 , 0 1})$. Die größten Effektstärken konnten dabei innerhalb der Skalen der anspannungsbezogenen Beschwerden ermittelt werden ( $\mathrm{ES}=0,85$ bis $\mathrm{ES}=1,63)$. Es zeigten sich schwache Korrelationen zwischen der Übungshäufigkeit im Alltag und der Belastung durch anspannungsbezogene Symptome zu T3 ( $r=+0,199 ; \mathrm{p}<0,01)$ sowie der Entwicklung anspannungsbezogener Beschwerden - besonders im Verlauf von T1 zu T3 $(r=-0,245 ; p<0,01)$. Wenig belastete Patienten oder solche, die eine besonders ausgeprägte Verbesserung ihrer Beschwerden erfuhren, neigten dazu, PR im Alltag etwas häufiger einzusetzen.

7. Übende vs. Nicht-Übende: Die Untersuchungen zur Symptomentwicklung in Abhängigkeit von der Übungshäufigkeit im Alltag ergaben, dass diejenigen Patienten, die mindestens einmal pro Woche übten, im Vergleich $\mathrm{zu}$ den nicht übenden Probanden signifikant weniger anspannungsbezogene Beschwerden zu T3 angaben $(p<0,01)$, eine signifikant stärkere Abnahme der Beschwerden von T1 zu T3 zeigten $(\mathrm{p}<0,01)$ und eine signifikant stärkere Veränderung des Wohlbefinden und Entspannungserlebens zu T2 und T3 ( $\mathrm{p}<0,01)$ erzielen konnten. Entsprechende Ergebnisse konnten im Rahmen des Vergleichs der extremen Quartile ermittelt werden.

8. Häufig Übende vs. regelmäßig Übende und Nicht-Übende vs. selten Übende: Die täglich übenden Patienten unterschieden sich von den Patienten, die ein- bis mehrmals pro Woche übten, lediglich hinsichtlich der Symptomentwicklung auf der Skala „körperliche und psychische Erschöpfung“ von T1 zu T3 (p<0,05). Die „Nicht-Übenden“ unterschieden sich von den „selten Übenden“ lediglich im veränderten Entspannungserleben zu T3 ( $\mathrm{p}<0,05)$. Die übrigen Vergleiche erbrachten keine signifikanten Resultate. 


\section{Diskussion}

Ziel der vorliegenden Studie ist es, einen Beitrag zur Verbesserung des Alltagstransfers von Progressiver Relaxation zu leisten. Günstige Bedingungen des Lern-, Übungs- und Transferprozesses sind die Voraussetzungen der präventiven und kurativen Wirkungen dieser Entspannungsmethode, die einen erfolgreichen und selbstständigen Einsatz im Lebensalltag überhaupt erst ermöglichen (Krampen, 2002b). Vor diesem Hintergrund sollen im Folgenden die dokumentierten Ergebnisse dieser Arbeit - auch im Vergleich mit der Pilotstudie von Arndt (2006) - diskutiert werden.

\subsection{Selbstständiges Üben während des Klinikaufenthalts und im Alltag}

Bereits Jacobson war sich der Notwendigkeit des kontinuierlichen Übens bewusst. Ein erfolgreiches „Selbst-Management“ mit dem Wissen um die eigenen Ressourcen und deren ökonomischem Einsatz erfordert Unabhängigkeit und Selbstverantwortung einerseits sowie die konsequente Übungsroutine andererseits. Tägliches Üben stellte für ihn die Voraussetzung einer erfolgreichen Umsetzung dar. Gleichzeitig betonte Jacobson die Integration der Entspannung in den Alltag, um einen generell entspannteren Lebensstil zu erlernen (Jacobson, 2006). Auch Bernstein und Borkovec, Begründer der modifizierten Versionen der Progressiven Relaxation, empfahlen das zweimalige Üben täglich - wenngleich in kürzeren Phasen als es Jacobson vertrat (Bernstein und Borkovec, 1975).

Auch vergleichsweise jüngere Studien unterstreichen die Bedeutsamkeit des kontinuierlichen Übens: Carlson und Hoyle (1993) konnten in ihrem Review die Wirksamkeit dieser Entspannungsmethode in der Therapie psychophysiologischer und Stress-abhängiger Störungen bestätigen - betonten jedoch, dass das Ausmaß der klinischen Wirksamkeit besonders von der Anzahl der Unterrichtstunden und der Dauer des Kurses positiv beeinflusst wird. Die Entwicklung spezifischer Fähigkeiten zur Selbstregulation und somit die Wirksamkeit der Methode korrespondierten mit der Intensität der Übungsroutine. Hillenberg und Collins (1983) kamen zu dem Ergebnis, dass sich besonders durch regelmäßiges Entspannungstraining Beschwerden wie Ängstlichkeit und Anspannung effektiv reduzieren lassen. 
Auch die Pilotstudie von Arndt aus dem Jahre 2005/2006 konnte den Zusammenhang zwischen der Übungshäufigkeit während des Rehabilitationsaufenthalts und einem erfolgreichen Alltagstransfers bestätigen ( $r=0,344 ; \mathrm{p}<0,01)$.

Bevor ein Vergleich zwischen den beiden Studien in Bezug auf die Übungshäufigkeit während des Klinikaufenthalts und schließlich im Alltag gezogen wird, soll im Folgenden zunächst der Studienaufbau hinsichtlich teilnehmender Probanden analysiert und diskutiert werden. Eine Übersicht liefert die folgende Abbildung (Abb.11). Die Prozentzahlen beziehen sich dabei auf die Gesamtheit der Patienten mit PR-Indikation zum Zeitpunkt T0.

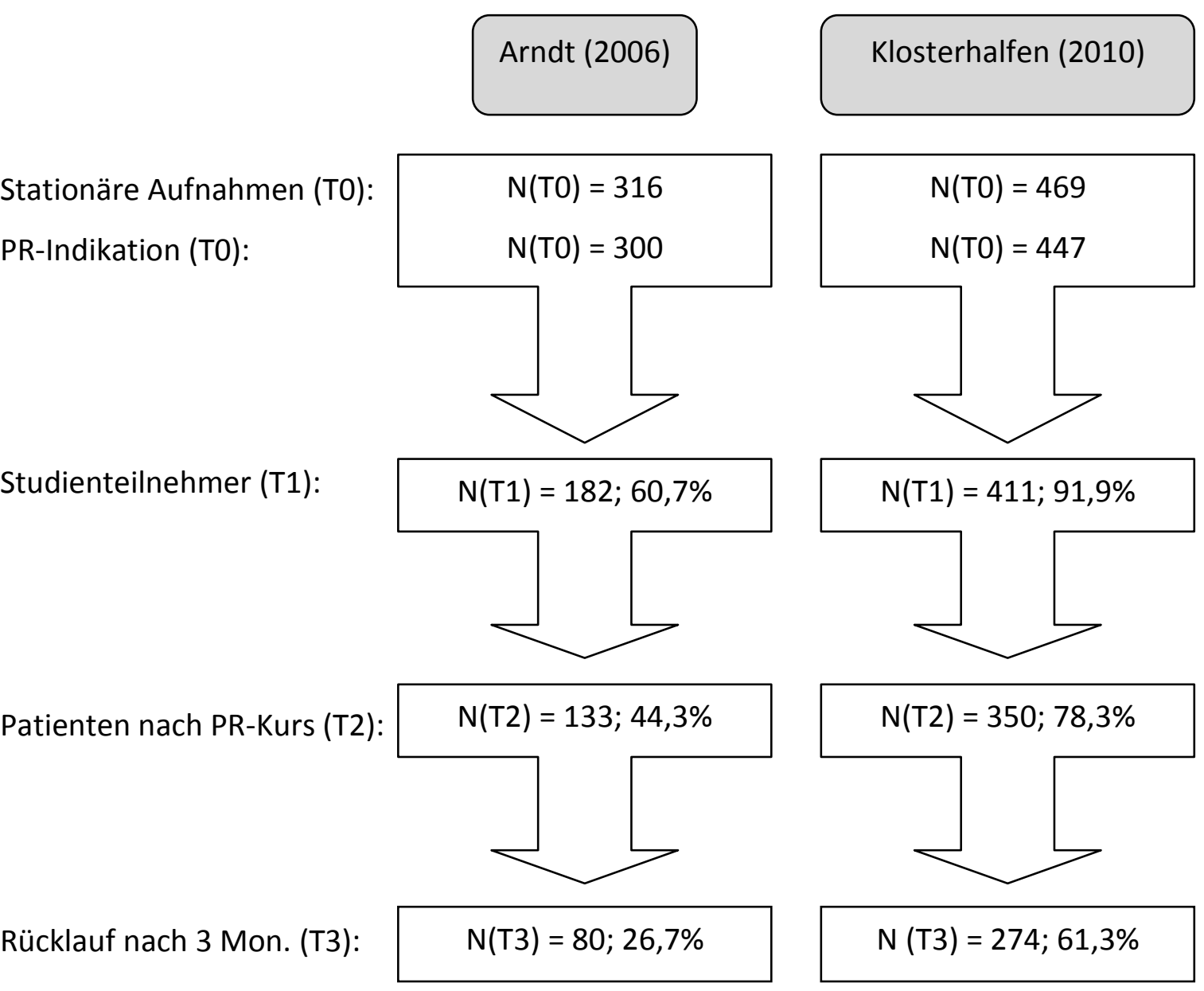

Abb.11: Übersicht über Studienteilnehmer und Drop-out-Quoten der beiden zu vergleichenden Studien

Die Schwächen der Pilotstudie, die schließlich Anlass zu einer Nachfolgestudie mit verbessertem Datensatz gaben, liegen zum einen in der mangelnden Repräsentativität der Stichprobe und zum andern in der fehlenden detaillierten Drop-out-Analyse. Es liegen keinerlei Angaben darüber vor, bei wie vielen Patienten der 316 stationären Aufnahmen die Indikation zur Teilnahme am PR-Kurs überhaupt gestellt wurde. Nach klinischer Erfahrung und Drop-out-Ana- 
lyse der vorliegenden Arbeit kann davon ausgegangen werden, dass bei ca. 5\% aller stationären Aufnahmen die Indikation zur Progressiven Relaxation nicht gegeben war. Da sich somit von den etwa 300 stationären Patienten der psychosomatischen Rehabilitationsklinik nur 60,7\% zur Teilnahme an der Studie bereiterklärten, ist davon auszugehen, dass vor allem solche Patienten eingeschlossen wurden, die generell Interesse an dieser Entspannungsmethode hatten, über gewisse Vorerfahrungen verfügten oder mit einer verstärkten Motivation sich dem PR-Kurs und somit der Studie anschlossen. Vor diesem Hintergrund müssen die verbleibenden 26,7\% zum Zeitpunkt T3 als selektive Stichprobe angesehen werden. Insgesamt musste eine Drop-out-Quote von 102 Patienten (56\%) im Verlauf von T1 zu T3 verzeichnet werden. Eine genaue Analyse, warum diese Patienten im Verlauf des Kurses und nach drei Monaten ausschieden, konnte jedoch nicht erfolgen.

Vor diesem Hintergrund versuchte die vorliegende Studie diese Schwächen zu minimieren: Zum einen kann von einer repräsentativen Stichprobe gesprochen werden, da sich von 447 Patienten mit Indikation zum PR-Kurs immerhin 91,9\% zur Teilnahme an der Studie bereiterklärten. Zum anderen konnten die Gründe des Ausscheidens durch Befragung der entsprechenden Studienteilnehmer und genaue Dokumentation im Rahmen einer detaillierten Drop-out-Analyse eruiert werden (s. Kapitel 3).

Krampen (2004) gibt eine Drop-out-Quote von 20-35\% bei PR-oder AT-Einführungskursen an. Auch Baider et al. (1994), die die PR-und GI-Wirkungen bei Krebspatienten untersuchten, mussten von Beginn bis zum Ende des Entspannungskurses eine Drop-out-Quote von $30,1 \%$ verbuchen. Weitere 32,6\% der verbliebenen Patienten stiegen bis zur katamnestischen Befragung nach sechs Monaten aus, sodass insgesamt eine Drop-out-Quote von 52,8\% erfasst wurde (Baider et al., 1994). In der vorliegenden Studie wurde dieser Anteil minimiert: Von 411 Patienten zu Beginn des Kurses schieden bis Abschluss des Entspannungstrainings lediglich 14,8\% (61 Patienten) aus. Von Beginn des stationären Aufenthalts bis zur Katamnese nach drei Monaten umfasste die Drop-out-Quote insgesamt nur 33,3\% (137 Patienten).

Dabei ist die recht hohe Rücklauf-Quote nach drei Monaten relevant: Von 350 Patienten nach Ende des PR-Kurses schickten immerhin 274 Studienteilnehmer die ausgefüllten Fragebögen nach drei Monaten zurück (78,3\%). In Anbetracht der Tatsache, dass die Katamnese dabei drei Fragebögen (AT-SYM, AT-VFE und AT-KATAM) mit insgesamt neun Seiten umfasste, ist der Rücklauf beachtlich - sodass auch zum Zeitpunkt T3 weiterhin von einer repräsentativen Stichprobe auszugehen ist. Vor diesem Hintergrund sollen nun die Ergebnisse der aktuellen Studie diskutiert werden. 
Übungshäufigkeit während des Klinikaufenthalts. Ein Entspannungskurs sollte nicht nur auf die Vermittlung eines Entspannungsverfahrens ausgerichtet sein, sondern auch gezielt der Motivationsförderung dienen (Ströbl et al., 2004). Daher sollten die Patienten auch im Rahmen des modifizierten Entspannungskurses regelmäßig zum selbstständigen Üben außerhalb der Kursstunden aufgefordert werden. Etwaige Probleme bei der eigenen Übungsroutine können so früher erkannt, ein individueller Übungsrhythmus bereits schon in der Klinik aufgebaut und der Einstieg in einen persönlichen Übungsprozess erleichtert werden.

69,4\% der Rehabilitanden (243 der 350 Studienteilnehmer, die den PR-Kurs absolvierten; konservative Schätzung) waren in der Lage, die erlernten Entspannungsübungen in ihren Klinikalltag zu integrieren. Nach optimistischer Schätzung konnte kein signifikanter Unterschied zwischen den beiden zu vergleichenden Studien hinsichtlich eines erfolgreichen Kliniktransfers festgestellt werden. Nach konservativer Schätzung übte jedoch ein signifikant höherer Anteil der Studienteilnehmer von 2010 auch außerhalb der Kursstunden. Die

\section{Hypothese 1 kann somit durch die vorliegenden Ergebnisse bestätigt werden.}

Zum einen legen diese Ergebnisse nahe, dass nicht alle Patienten von Progressiver Relaxation profitieren - diese Rehabilitanden würden eventuell mit anderen Entspannungstechniken bessere Erfolge erzielen. Zum anderen bestehen weiterhin Verbesserungsmöglichkeiten zukünftiger Kurse. Es blieb daher zu analysieren, ob das selbstständige Üben auch außerhalb des PRKurses vom Kursleiter überhaupt thematisiert und angeregt wurde, wovon diese Erfolgsquote abhängt bzw. wodurch diese noch gesteigert werden kann.

Die Patienten wurden im Rahmen des Katamnesefragebogens befragt, wie häufig sie der Kursleiter zum selbstständigen Üben aufforderte und inwieweit die Gelegenheit bestand, im Kurs über die Erfahrungen beim selbstständigen Üben zu sprechen. 82,1\% (Motivation zum regelmäßigen Üben) bzw. 66,1\% (Erfahrungsaustausch) der Befragten betonten die RegelmäBigkeit. Von Seiten des Kursleiters wurden die Bedingungen des modifizierten Entspannungskurses laut Angaben der Kursteilnehmer somit ganz überwiegend erfüllt. Einerseits könnte eine Verzerrung der Angaben durch sozial erwünschtes Antwortverhalten (Möhring und Schlütz, 2010) eine mögliche Erklärung dafür sein, das manche Patienten davon abhielt, sich kritisch gegenüber den ihnen bekannten Kursleitern zu äußern. Andererseits scheint es jedoch auch wohl bedeutsamere Faktoren zu geben, die die Übungshäufigkeit während des Klinikaufenthalts beeinflussen. Die Korrelation der Übungshäufigkeit während des Klinikaufenthalts mit dem veränderten Entspannungserleben zu T2 und der Symptombelastung zu Beginn der Rehabilitationsmaßnahme zeigte zumindest schwache Zusammenhänge. Demnach übten jene Patienten während des Klinikaufenthalts häufiger, die bereits im 
Laufe des Kurses eine Verbesserung ihres Entspannungserlebens bemerkten und/ oder zu Beginn ihres Reha-Aufenthalts (auf bestimmten Skalen) besonders belastet waren.

Eventuell ging mit dem Ausmaß der anspannungsbezogenen Beschwerden zu T1 eine ausgeprägte Motivation einher, die Entspannungsübungen umsetzen zu wollen. Zudem kommt dem initialen Gefühl eines veränderten Entspannungserlebens durch Progressive Relaxation somit nicht nur im Hinblick auf einen erfolgreichen Alltagstransfer (s.Kap.5.2), sondern auch hinsichtlich einer kontinuierlichen Übungsroutine bereits während der Reha eine besondere Bedeutung zu.

In diesem Zusammenhang dürfen gerade die Umgebungsbedingungen nicht außer Acht gelassen werden. Bereits im Jahre 1985 konnten Krampen und Ohm die subjektive Wahrnehmung der Behandlungsumgebung als wichtige psychosoziale Variable in Bezug auf des Wohlbefinden und die Genesung von Reha-Patienten nach Myokard-Infarkt identifizieren. Zum Zeitpunkt der Datenerhebung fanden in der Klinik umfangreiche Umbaumaßnahmen statt, die möglicherweise einige Patienten davon abhielten, sich auf erste Übungsschritte im persönlichen Umfeld einzulassen. Untersuchungen nach Abschluss des Umbaus könnten wiederum andere Ergebnisse mit eventuell höherer Erfolgsquote liefern. Die Schaffung einer angenehmen Übungsatmosphäre in der Klinik könnte das Erlernen von Entspannung und Veränderung durch PR intensivieren und somit zu einer verstärkten Trainingstätigkeit bereits während des Klinikaufenthalts führen - welche wiederum dem erfolgreichen Alltagstransfer zugute kommt (s. Kap. 5.2).

Übungshäufigkeit im Alltag nach drei Monaten. Im Rahmen des modifizierten Entspannungskurses gaben die 274 verbleibenden Studienteilnehmer eine durchschnittliche Übungshäufigkeit von mehrmals im Monat an. Nach optimistischer Schätzung konnten $\mathbf{5 0 , 4 \%}$ das Gelernte erfolgreich in ihren individuellen Alltag integrieren. Im Jahre 2006 ermittelte Arndt einen Anteil von 52,5\% mit erfolgreichem Alltagstransfer und somit einer Übungshäufigkeit von mindestens einmal pro Woche. Weder nach optimistischer noch nach konservativer Schätzung konnte ein Unterschied zwischen den beiden zu vergleichenden Studien erfasst werden. Die Hypothese 2 muss somit verworfen werden.

Vor diesem Hintergrund muss jedoch auf die prinzipiell eingeschränkte Vergleichbarkeit der beiden Studien hingewiesen werden. Eine repräsentative Stichprobe zu allen drei Messzeitpunkten mit guter Rücklaufquote zu T3 steht einer eher selektiven Stichprobe (zumindest nach drei Monaten) gegenüber. Da in der Pilotstudie letztlich nur 44\% der eingeschlossenen Studienteilnehmer bzw. ein Viertel der Gesamtheit aller stationären Aufnahmen die 
Katamnesefragebögen zurückschickte, kann von einer Verzerrung in die positive Richtung ausgegangen werden: Es antworteten vor allem jene Patienten, die von Anfang an positive Erfahrungen mit dieser Entspannungsmethode sammelten. Plakativ gesagt könnte dies bedeuten, dass diejenigen Patienten, die zu T3 gar nicht erst antworteten, am Transfer des Gelernten in den Alltag scheiterten.

Das Ziel zukünftiger Kurse müsste es sein, diese Erfolgsquote von 50,4\% zu steigern. Ein Schritt zur Verbesserung des Alltagstransfers von Progressiver Relaxation kann es daher sein, mögliche Prädiktoren zu erfassen, die die Übungswahrscheinlichkeit zuhause vorhersagen, um die Bedingungen zukünftiger Entspannungskurse zu optimieren. Mögliche Prädiktoren und deren Konsequenzen für zukünftige Kurse werden im nachfolgenden Kapitel diskutiert. 


\subsection{Prädiktoren eines erfolgreichen Alltagstransfers von PR}

Im Jahre 2002 konnte Krampen bereits einige Prädiktoren des erfolgreichen Erwerbs und gelungenen Alltagstransfers von Progressiver Relaxation und Autogenem Training ermitteln. Dabei standen sowohl Vorerfahrungen mit Entspannungstechniken als auch Teilnahmemotive im Vordergrund. Während beim AT der Lern- und Transfererfolg am wahrscheinlichsten bei Probanden ohne Vorerfahrungen oder mit AT-Kenntnissen war, konnte für Progressive Relaxation keine signifikanten Unterschiede zwischen Studienteilnehmer unterschiedlichen Kenntnisstandes errechnet werden. Es wurde jedoch belegt, dass Patienten mit Vorerfahrungen in anderen Entspannungstechniken häufiger PR-Kurse abbrachen. Desweiteren konnte eine Schwellenhypothese formuliert werden: Vor allem intrinsische Teilnahmemotive korrelierten mit einem erfolgreichen Alltagstransfer bis zu einer Motivanzahl von vier Motiven. Die Vorhersage von Übungswirkungen und Kursabbrüchen von Progressiver Relaxation konnte somit anhand von Vorerfahrungen erfolgen (Krampen, 2002; Krampen und von Eye, 2008). Auch Bernardy et al. konnten im Jahre 2008 intrinsische Teilnahmemotive und eigenständiges Üben als signifikante Prädiktoren eines erfolgreichen und langfristigen Transfers identifizieren. Ströbl et al. (2004) und Reusch und Ströbl (2004) erfassten in ihren Studien unterschiedliche Motivationslagen und -stufen bei Teilnehmern von Entspannungskursen und hoben die Bedeutung der Selbstwirksamkeitserwartung und der wahrgenommenen Vorteile des umgesetzten Gesundheitsverhaltens hervor.

Im gleichen Jahr baute Krampen auf seinen Ergebnissen auf und plädierte für ein mehrstufiges differenzialdiagnostisches Vorgehen, um die differentielle Indikation von Autogenem Training und Progressiver Relaxation zu optimieren: Nach einer allgemeinen Indikationsstellung schließt sich die Erfassung von Vorerfahrungen und Teilnahmemotiven an, bevor im letzten Schritt änderungssensitive Symptome erfasst werden sollen. Progressive Relaxation erwies sich diesbezüglich als Methode der ersten Wahl bei erhöhter Nervosität und innerer Anspannung (Krampen, 2004).

Eingebettet in diese Vorbefunde sollten im Rahmen der vorliegenden Studie zum einen weitere Prädiktoren erfasst werden, um einen erfolgreichen Alltagstransfer vorhersagen zu können und gegebenenfalls die Bedingungen zukünftiger Kurse zu optimieren. Zum anderen wurden - basierend auf dem mehrstufigen differenzialdiagnostischen Vorgehen nach Krampen - änderungssensitive und anspannungsbezogene Symptome abgefragt und ausgewertet, um die Wirkung der Entspannungsmethode auf etwa bestehende Beschwerden (auch in Abhängigkeit von der Übungshäufigkeit) objektivieren zu können. Im Folgenden werden mögli- 
che Prädiktoren diskutiert, bevor im nächsten Kapitel (5.3) Stellung zu einer möglichen Symptomentwicklung genommen wird.

Prädiktor 1: Übungshäufigkeit während des Klinikaufenthalts. Es zeigte sich eine mittelgradige Korrelation zwischen der Übungshäufigkeit im Alltag nach drei Monaten und der Übungshäufigkeit während des Klinikaufenthalts außerhalb der Kursstunden $(\mathbf{r}=+\mathbf{0 , 5 5 8}$; $\mathrm{p}<0,01)$. Eigenständiges Üben konnte hier - wie nach den Arbeiten von Arndt (2006) und Bernardy et al. (2008) erwartet - ebenfalls als Prädiktor eines erfolgreichen Alltagstransfers identifiziert werden. Auch bei der gemeinsamen Betrachtung im Rahmen der linearen Regressionsanalyse blieb der Effekt dieser Variable signifikant (Beta=0,506; p<0,01). Die Hypothese 3a wurde somit bestätigt. Der Motivation der Übungsteilnehmer zum selbstständigen Üben bereits während des Klinikaufenthalts durch den Kursleiter kommt daher auch in $\mathrm{Zu}$ kunft entscheidende Bedeutung zu. Die Entspannungstherapeuten sollten gerade der Besprechung individueller Erfahrungen beim Üben besondere Aufmerksamkeit beimessen, um den Weg zu einem erfolgreichen Erwerb und Transfer dieser Entspannungsmethode zu ebnen. Darüber hinaus könnte die Ausweitung des Entspannungstrainings auf mehrere Sitzungen oder das Bereitstellen entsprechender Räume zum gemeinsamen Üben auch außerhalb der Kursstunden die Übungsroutine fördern. Ziel des Entspannungskurses muss es sein, den Übungsteilnehmern den Einstieg in eine individuelle Übungsroutine zu erleichtern. Da nach Abschluss einer Rehabilitationsmaßnahme und Rückkehr in den persönlichen Alltag meist einige Veränderungen und Herausforderungen auf die Rehabilitanden warten, wäre es wünschenswert, ihnen zum Start in die „Zeit nach der Reha“ die Progressive Relaxation als Stütze im Alltag mitzugeben. Dafür müssen jedoch während der Reha die Bedingungen für das Erleben der positiven Wirkungen und Veränderungen durch Entspannung gegeben sein.

Prädiktor 2: Das veränderte Entspannungserleben. Die Ergebnisse belegen, dass die Studienteilnehmer nach Abschluss des Kurses eine positive Veränderung wahrnehmen, die auch nach drei Monaten noch nachweisbar ist-wenn auch in abgeschwächter Form. Dabei schlägt sich die erfahrene Entwicklung am stärksten auf anspannungs- bzw. entspannungsbezogenen Skalen und im allgemeinen Wohlbefinden nieder.

Zum einen sprechen diese Veränderungen für die anhaltend entspannungsfördernden Wirkungen des PR-Kurses. Zum anderen müssen diese Ergebnisse im Kontext anderer Therapiemodule gesehen werden. Die Kursteilnehmer wurden regelmäßig angewiesen, die Entwicklungen jeweils in Bezug auf den Entspannungskurs einzuschätzen. Zudem wurden statt 
allgemeiner Symptomchecklisten Messinstrumente eingesetzt, die speziell anspannungsbezogene Beschwerden erfassen. Die Möglichkeit des Konfusionseffekts mit anderen Therapiemodulen bleibt jedoch bestehen. Um diesen Effekt $\mathrm{zu}$ minimieren, müsste der Entspannungsgruppe eine Kontrollgruppe ohne PR-Training während der Rehabilitation gegenübergestellt werden. Nur auf diese Weise könnte herausgefunden werden, inwieweit die erlebten Veränderungen auf den Entspannungskurs zurückzuführen sind.

Die Mittelwerte dieser 12 Items zu T2 (r=-0,240; $\mathrm{p}<0,01)$ und T3 (r=-0,356; $\mathrm{p}<0,01)$ korrelierten schwach bis mittelgradig mit der Übungshäufigkeit nach drei Monaten. Bei der gemeinsamen Betrachtung im Rahmen der linearen Regressionsanalyse blieb der Effekt der Variable „verändertes Entspannungserleben zu T3“ signifikant (Beta=-0,275; p<0,01). Das veränderte Entspannungserleben nach drei Monaten kann daher als Prädiktor eines erfolgreichen Alltagstransfers angesehen werden. Die Hypothese $3 b$ bestätigte sich also.

Dieses Ergebnis lässt vielfältige Schlüsse zu. Zum einen unterstreichen die Resultate die nachhaltige Wirkung der Progressiven Relaxation - wenngleich der soeben beschriebene Konfusionseffekt nicht ausgeschlossen werden kann. Zum anderen kann man daraus Konsequenzen für zukünftige Entspannungskurse ableiten. Sowohl Edmund Jacobson als auch seine „Nachfolger“ Bernstein, Borkovec und Öst betonten im Sinne der „differenziellen Entspannung“ den Transfer der Entspannungsübungen auf Aktivitäten des Alltags. Die Patienten sollten fähig sein, die erlernten Techniken auch während alltäglicher Tätigkeiten - also sowohl im Liegen, Sitzen als auch Umherlaufen - anzuwenden und davon zu profitieren (Derra, 2007). Darauf aufbauend zeigt die klinische Erfahrung, dass im Rahmen von Entspannungskursen - so auch in Blieskastel - der Alltagstauglichkeit besondere Bedeutung beigemessen wird und die Kursteilnehmer dazu aufgefordert werden, die Übungen auch in alltagsnäheren Situationen durchzuführen. Einerseits fördert diese Ausrichtung einen möglichen Alltagstransfer dadurch, dass relativ früh Entspannung auch in weniger angenehmen Situationen trainiert wird. Andererseits zeigt uns dieses Ergebnis, dass besonders das initiale Gefühl der Veränderung wichtig ist, um den Nutzen des Trainings zu erkennen und in eine bestenfalls tägliche Übungsroutine umzuwandeln. Der Aufbau und die Bedingungen eines Entspannungskurses sollten daher dem Patienten erst mal ermöglichen, eine Veränderung des seelischen und körperlichen Wohlbefindens überhaupt wahrzunehmen. Dies kann durch das Schaffen geeigneter Rahmenbedingungen wie beispielsweise einer angenehmen Atmosphäre, Üben im Liegen und individuelle Betreuung gelingen. Wie bereits geschildert, konnte dem veränderten Entspannungserleben auch ein Einfluss auf die Übungshäufigkeit während des Klinik- 
aufenthalts zugeschrieben werden. Das Erleben von Entspannung und Veränderung hat somit kurzfristige als auch langfristige positive Auswirkungen.

Ein Entspannungskurs sollte beides vereinen: das initiale Gefühl eines veränderten Wohlbefindens durch Entspannung überhaupt erst erleben lassen und im zweiten Schritt das Üben unter alltagsnahen Bedingungen trainieren. Die Motivation eines Individuums ist abhängig von den Wechselwirkungen zwischen persönlichen Bedürfnissen bzw. Präferenzen und situativen Anreizen (Heckhausen und Heckhausen, 2006). Erst wenn am eigenen Körper erlebt wird, dass sich durch Progressive Relaxation etwas am persönlichen Wohlbefinden verändert - also verbessert - ist die Motivation groß genug, diesen Effekt auch unter weniger angenehmen (Alltags-) Gegebenheiten zu erzielen.

Prädiktor 3: Bewertung der Gruppengröße. Während Edmund Jacobson das Einzelsetting favorisierte, steht spätestens seit Bernstein und Borkovec das Gruppensetting im Fokus des Interesses (Bernstein und Borkovec, 1975) - welches auch heutzutage in den meisten Rehabilitationskliniken so umgesetzt wird. Im Rahmen der Konsensuskonferenzen zur Progressiven Relaxation und deren Anwendung im stationären und ambulanten Bereich wurde für geschlossene Gruppen (nach individueller Einführung auch teiloffene Gruppen) bis maximal 12 Personen (bei Kindern ab ca. sechs Jahren bis maximal sechs, ab zehn Jahren bis maximal acht Teilnehmer) plädiert (Ohm, 2004). Der ursprüngliche Entspannungskurs der ArndtStudie in Blieskastel umfasste Gruppen von etwa 8-12 Personen. Beim modifizierten Kurs lag die durchschnittliche Teilnehmerzahl ebenfalls zwischen acht und zwölf Personen, wobei auch „Ausreißergruppen“von fünf bis zu 25 Patienten vorkamen.

Die durchschnittliche Bewertung der Gruppengröße nach Ende des Entspannungskurses lag zwischen „etwas zu groß (2)“ (nach bipolarer Skala) und „angemessen (3)“ und korrelierte nicht mit der Übungshäufigkeit nach drei Monaten ( $r=-0,056 ; \mathrm{p}=0,358 ; \mathrm{n}$. s.). Die Bewertung der Gruppengröße blieb somit ohne Einfluss auf den Alltagstransfer. Die Hypothese 3c ließ sich nicht bestätigen.

Die klinische Erfahrung ließ dabei eher das Gegenteil vermuten, da die Gruppengröße im persönlichen Gespräch oftmals als Kritikpunkt zur Sprache kam. Darüber hinaus nutzen einige Patienten die freien Zeilen am Ende des Veränderungs- und Katamnesefragebogens, um ihrem Wunsch nach kleineren Gruppen Ausdruck zu verleihen. Somit darf auch bei dieser Bewertung das Antwortverhalten nach dem Phänomen der sozialen Erwünschtheit (Möhring und Schlütz, 2010) nicht gänzlich ausgeschlossen werden. Kleinere Gruppen bieten die Möglich- 
keit zur intensiveren Betreuung der einzelnen Kursteilnehmer, sind nach diesen Ergebnissen jedoch nicht zwangsläufige Voraussetzung erfolgreicher Entspannungskurse.

Prädiktor 4: Benotung des Entspannungstrainings. Die Benotung des Entspannungstrainings korrelierte nur schwach mit einem erfolgreichen Alltagstransfer $(r=+0,243 ; \mathrm{p}<0,01)$. Die Hypothese 3d konnte somit nur bedingt bestätigt werden. Die positive Bewertung des Entspannungskurses scheint demnach einen positiven Einfluss auf den Alltagstransfer zu haben. Bei der gemeinsamen Betrachtung im Rahmen der linearen Regressionsanalyse war der signifikante Effekt jedoch nicht mehr nachweisbar.

Durchschnittlich verliehen die Studienteilnehmer dem Kurs der Progressiven Relaxation die Note 1,97, also eine „gute“ Zwei. Dies ist zweifelsohne ein wortwörtlich gutes Ergebnis, im Auge des ehrgeizigen Betrachters jedoch verbesserungswürdig. Der Entspannungskurs beinhaltet neben den eigentlichen Übungsstunden das Grundlagenseminar „Entspannung“ sowie die Ausgabe von Übungsmaterialien in schriftlicher Form (Übungstexte). Um ein mögliches Verbesserungspotenzial zu eruieren, wurde die Bewertung dieser Bestandteile des Entspannungskurses analysiert. Die Kursteilnehmer empfanden den Inhalt des Grundlagenseminars als seiner Hörerschaft angemessen. Der Vortragsstil des Entspannungstherapeuten - wobei zu beachten ist, dass auch hin und wieder ein Wechsel des Vortragenden stattfand - erhielt eine „gute“ Zwei (MW=1,84, SD=0,81), die ausgeteilten Übungstexte wurden durchschnittlich mit einer „Zwei Minus“ (MW=2,44, SD=1,03) bewertet.

Die Ergebnisse verdeutlichen, dass neben dem eigentlichen Entspannungskurs auch der Vorbereitung der Kursteilnehmer (im Sinne eines solchen Grundlagenseminars) und der zusätzlichen Hilfsmaterialen eine gewisse Bedeutung beizumessen ist. Die Übungstexte sollten möglichst allen Patienten ausgehändigt und deren Anwendung auch im Rahmen des Entspannungskurses diskutiert werden. Der Kursleiter soll die lernenden und übenden Kursteilnehmer auf ihrem Weg zur systematischen Entspannung begleiten (Stetter, 2004) und sich dessen bzw. der Bedeutung seiner Rolle auch bewusst sein.

Darüber hinaus dürfen die persönlichen Verbesserungsvorschläge der Patienten nicht außer Acht gelassen werden. Auffällig ist, dass insgesamt 45,3\% der Kursteilnehmer den Wunsch nach einer CD äußerten. Dies kann ein durchaus vielversprechender Ansatz sein, kommende Entspannungskurse zu verbessern und die Betreuung der Kursteilnehmer - auch über die Zeit der Reha hinaus - zu intensivieren und optimieren. 
Prädiktor 5: Alter und Geschlecht. Die Korrelation der Übungshäufigkeit nach drei Monaten mit diesen soziodemographischen Variablen erbrachte keine relevanten Ergebnisse: Die sehr gering ausgeprägte Korrelation mit dem Alter wurde aufgrund der großen Stichprobe zwar statistisch signifikant $(\mathrm{r}=-0,127 ; \mathrm{p}<0,05)$; dieser signifikante Effekt ging jedoch im Rahmen der linearen Regressionsanalyse zurück. Das Geschlecht hatte keinerlei Einfluss auf die Übungshäufigkeit zuhause. Die Hypothese 3e muss somit hinsichtlich des Geschlechts verworfen werden und auch die Korrelation mit dem Alter ist praktisch nicht relevant.

Das Geschlecht lässt keine konkreten Vorhersagen zu, inwieweit die erlernten Entspannungsübungen in den Alltag integriert werden können. Die Resultate sprechen jedoch dafür, dass es älteren Patienten etwas leichter fällt, auch über die Zeit des Klinikaufenthalts hinaus von den Entspannungsübungen zu profitieren. Dabei muss jedoch beachtet werden, dass bei den hier dokumentierten Kursen weniger als $15 \%$ der Kursteilnehmer unter 40 Jahren alt waren und somit der Anteil junger Patienten sehr gering ist. Die durchschnittliche Übungshäufigkeit der Patienten $\leq 40$ Jahren und der Patienten $>40$ Jahren unterschied sich jedoch nicht signifikant voneinander ( $t=0,584 ;$ n. s.). Für zukünftige Programme bedeutet dies, dass die zufällige Verteilung der Patienten - ungeachtet des Alters oder Geschlechts - auf die einzelnen Kurse beibehalten werden kann. Es bedarf beispielsweise keiner speziellen Förderung bestimmter Altersgruppen hinsichtlich individueller Übungsroutine.

Prädiktor 6: Aktuelles subjektives Wohlbefinden. Das aktuelle subjektive Wohlbefinden zum Zeitpunkt T1 lag im Mittel bei 4,24 \pm 1,48 - wobei 1 als ,sehr unwohl“ und 7 als ,sehr wohl“"klassifiziert wurde. Es zeigte sich eine schwache Korrelation dieser Variablen mit der Übungshäufigkeit im Alltag ( $r=-0,165$; $\mathrm{p}<0,01)$. Die Hypothese $3 f$ konnte nicht bestätigt werden. Auch wenn dieser signifikante Effekt im Rahmen der gemeinsamen Betrachtung der linearen Regression zurückging lässt sich daraus schließen, dass besonders diejenigen Patienten auch drei Monate nach Ende des Kurses die Entspannungsübungen erfolgreich im Alltag praktizieren, denen es zu diesem Zeitpunkt besonders gut geht. Die Abhängigkeit des Alltagstransfers vom aktuellen Beschwerdebild findet sich ebenfalls bei der Korrelation der alltäglichen Übungshäufigkeit mit der Belastung durch anspannungsbezogene Symptome (s. Kap. 4.4 und Kap. 5.3). Der Stabilisierung und weiteren Verbesserung des Beschwerdebildes etwa durch Nachsorgeprogramme oder fortgesetzte ambulante Gruppen- oder Einzeltherapie kommen daher auch in Bezug auf den erfolgreichen Alltagstransfer von Entspannungstechniken besondere Bedeutung zu. Die Motivation zum Üben steht und fällt anscheinend mit der situativen Belastung durch seelische Faktoren oder auch körperlich bedingte, anspannungsbe- 
zogene Beschwerden. Gleichzeitig bedeutet dieses Ergebnis jedoch, dass sich die PR als Stütze gerade in Zeiten erhöhter Anspannung und gesteigerten Unwohlseins nicht durchsetzen konnte. Entspannung nur in Zeiten grundsätzlichen Wohlbefindens war nicht die ursprüngliche Intention des „PR-Vaters“ Jacobson. Progressive Relaxation sollte so „verinnerlicht“ sein, dass sie gerade in Phasen erhöhter Belastung Anwendung findet und ihre kurativen Wirkungen entfalten kann. Dieses Resultat unterstreicht die Notwendigkeit der Motivation zum Üben auch oder gerade in Situationen erhöhter Anspannung. Zukünftige Kursleiter sollten diesen Zusammenhang besprechen und die Bedeutung der PR als „eingebauten Tranquilizer“ (Jacobson, 2006) in hektischen Zeiten betonen. 


\subsection{Zusammenhang zwischen der Übungshäufigkeit im Alltag und der Symptombelastung bzw. eines veränderten Entspannungserle- bens}

Die klinische Wirksamkeit der Progressiven Relaxation ist anerkannt und durch vielfältige Arbeiten belegt (Stetter, 2004) - sowohl in Bezug auf chronische Schmerzzustände, somatische Beschwerden wie essentielle Hypertonie, bronchopulmonale, gastrointestinale und maligne Erkrankungen, als auch in der Therapie psychischer Störungen (s.Kap.2.3). Gerade für den Bereich der Rehabilitation existieren diesbezüglich einige Studien (Van Dixhoorn und White, 2005; Hui et al., 2006).

Die vorliegende Studie liefert hypothesenkonform einen weiteren Nachweis der klinischen, nachhaltigen Wirksamkeit von Progressiver Relaxation im Rahmen der psychosomatischen Rehabilitation: Die aktuellen Beschwerden der Studienteilnehmer hinsichtlich körperlicher und psychischer Erschöpfung, Nervosität und innerer Anspannung, psychophysiologischer Dysregulation, Leistungs- und Verhaltensschwierigkeiten, Schmerzbelastung und Problemen mit Selbstbestimmung und Selbstkontrolle wurden auf sechs Skalen mit jeweils acht Items zu drei verschiedenen Messzeitpunkten erfasst. Dabei nahmen die Symptome im Verlauf des Klinikaufenthalts auf allen Skalen hochsignifikant ab. Drei Monate nach Entlassung aus der Reha hatten die Beschwerden wieder leicht zugenommen - ohne den Ausgangswert zu erreichen. Die Symptome der katamnestischen Befragung unterschieden sich weiterhin hochsignifikant von jenen zum Zeitpunkt der stationären Aufnahme - die Hypothese 4a wurde somit bestätigt. Die größten Effektstärken konnten dabei innerhalb der Skalen der anspannungsbezogenen Beschwerden ermittelt werden.

Diese Ergebnisse unterstreichen also den nachhaltigen Effekt von Progressiver Relaxation. Aufgrund der Tatsache, dass keine Kontrollgruppe ohne Entspannungstraining existierte, kann jedoch ein Konfusionseffekt mit anderen Therapiemodulen nicht ausgeschlossen werden.

Im nächsten Schritt wurden sowohl die aktuellen Beschwerden zu T1, T2 und T3 als auch die Symptomentwicklung (mittels Differenzwerte) mit der Übungshäufigkeit im Alltag korreliert - wobei nun das Augenmerk speziell auf anspannungsbezogene Beschwerden (Skalen „körperliche und psychische Erschöpfung“, „Nervosität und innere Anspannung“) gelegt wurde. Dabei bestand ein hochsignifikanter Zusammenhang zwischen der Übungshäufigkeit nach drei Monaten und dem aktuellen Beschwerdebild zu T3 als auch der Symptomentwicklung von T1 zu T3. Die Hypothese 4b konnte diesbezüglich bestätigt werden. 
Zum einen betonen diese Ergebnisse die Abhängigkeit der „,alltäglichen“ Übungshäufigkeit vom situativen Wohlbefinden, zum andern heben sie die Bedeutung des Gefühls der Veränderung abermals hervor. Genau jene Patienten integrieren erfolgreich Entspannungsübungen in ihren Alltag, deren Wohlbefinden im Alltag besonders ausgeprägt ist und/oder deren Beschwerden sich im Verlauf merklich verändert haben.

Um gezielter erfassen zu können, welche Auswirkungen die Progressive Relaxation auf anspannungsbezogene Beschwerden hat, wurde das Patientenkollektiv in „Übende“ vs. „NichtÜbende“ eingeteilt bzw. die „extrem Übenden“ den „extrem Nicht-Übenden“ gegenüber gestellt. Zudem wurden die „häufig Übenden“ mit den „,regelmäßig Übenden“ und die „NichtÜbenden“ mit den „,selten Übenden“ verglichen. Die Ergebnisse konnten die Hypothesen 4c und 4d bestätigen: Diejenigen Patienten, die mindestens einmal pro Woche übten, gaben im Vergleich $\mathrm{zu}$ den nicht übenden Probanden signifikant weniger anspannungsbezogene Beschwerden zu T3 an ( $<<0,01)$, zeigten eine signifikant stärkere Abnahme der Beschwerden von T1 zu T3 $(\mathrm{p}<0,01)$ und konnten eine signifikant stärkere Veränderung des Wohlbefinden und Entspannungserlebens zu T2 und T3 $(\mathrm{p}<0,01)$ erzielen. Der Vergleich der extremen Quartile konnte diese Ergebnisse unterstreichen und lieferte entsprechende Resultate.

Interessant ist diesbezüglich der abschließende Vergleich „häufig vs. regelmäßig Übende“ und „Nicht-Übende vs. selten Übende“: Lediglich die Entwicklung (T1-T3) der anspannungsbezogenen Beschwerden der Skala „körperliche und psychische Erschöpfung“ der „häufig Übenden“ unterschied sich auf dem 5\%-Niveau signifikant von der der „regelmäßig Übenden“. Weder die aktuelle Beschwerdebelastung, die Symptomentwicklung von T2 zu T3 noch das veränderte Entspannungserleben nach drei Monaten zeigten einen signifikanten Unterschied zwischen diesen Gruppen. Dieses Ergebnis impliziert, dass sowohl die tägliche als auch die wöchentliche Übungshäufigkeit die Symptome und das Entspannungserleben im gleichen Ausmaß zu verbessern vermag. Anders ausgedrückt: Der erfolgreiche Alltagstransfer von mindestens einmal pro Woche genügt im Prinzip, um anspannungsbezogene Beschwerden zu lindern und ein verändertes Gefühl von Entspannung wahrzunehmen. Eine übermäBige, mehrmals tägliche Anwendung erzeugt diesbezüglich keinen zusätzlichen Benefit.

Dieses Resultat bietet eine gute Motivationsgrundlage zukünftiger Seminare und Kurse. Die Patienten sollten darüber informiert werden, dass sie selbst im Stande sind, bereits durch regelmäßige, aber nicht übermäßige Trainingseinheiten von diesen zu profitieren. Ihnen kann der Druck genommen werden, täglich üben zu müssen, um Erfolge zu verbuchen. Darüber hinaus bedarf jedoch auch eine tägliche Übungsroutine einer gewissen Zeitspanne, um ihre Wirkung zu entfalten. Es zeigen sich gewisse Unterschiede in der Symptomentwicklung hin- 
sichtlich „körperlicher und psychischer Erschöpfung“; diese treten jedoch auch erst im Verlauf von T1 zu T3 zu Tage.

Der Vergleich der „Nicht-Übenden“ mit den „,selten Übenden“ unterstreicht die Bedeutung der Kontinuität. Hinsichtlich Symptombelastung und Symptomentwicklung unterschieden sich die beiden Gruppen nicht signifikant voneinander. Es bestanden jedoch Unterschiede im veränderten Entspannungserleben und Wohlbefinden nach drei Monaten $(p<0,05)$. Plakativ gesagt: Diejenigen Patienten, die selten bis gar nicht übten, hatten nach drei Monaten ein ähnliches Beschwerdebild wie jene, die mindestens einmal im Monat die Entspannungsübungen anwandten. Eine monatliche Anwendung reicht aus, um ein verändertes Entspannungserleben und Wohlbefinden zu erfahren (Signifikanz auf dem 5\%-Niveau), genügt jedoch nicht, um die etwaigen Beschwerden signifikant zu beeinflussen.

Ein optimales Verhältnis zwischen (körperlichem und zeitlichem) Einsatz und Nutzen (im Sinne einer Symptomreduktion und eines veränderten Entspannungserlebens) bietet somit eine Übungsroutine von mindestens einmal wöchentlich.

Zusammenfassend lassen sich aus diesen Ergebnissen folgende Schlüsse ziehen:

Die klinische Wirksamkeit von Progressiver Relaxation konnte bestätigt werden. Die Beschwerden waren auf allen Skalen hochsignifikant rückläufig.

$\rightarrow$ Die Ergebnisse sprechen für die Nachhaltigkeit dieser Entspannungsmethode. Die Beschwerden waren auch noch nach drei Monaten hochsignifikant niedriger als zu Beginn der Rehabilitation.

$\rightarrow$ Im Sinne der Selbstwirksamkeit kommt der gefühlten Veränderung besondere Bedeutung zu. Besonders jene Patienten können die Entspannungsübungen erfolgreich in den Klinikalltag und persönlichen Alltag zuhause integrieren, die eine Verbesserung der Beschwerden erfahren (haben). Je größer die Veränderung bzw. Verbesserung ist, desto wahrscheinlicher ist ein erfolgreicher Alltagstransfer.

$\rightarrow$ Das Entfalten der positiven Auswirkungen von PR erfordert Zeit und regelmäßiges Üben. Die Unterschiede zwischen „Übenden“ und „Nicht-Übenden“ waren vor allem im Rahmen der katamnestischen Befragung signifikant. Im Verlauf des Klinikaufenthalts (T1-T2) bestand kein signifikanter Unterschied in der Symptomentwicklung oder im Beschwerdebild - dieser trat erst drei Monate nach Abschluss der Reha, also nach längerfristiger Anwendung, zu Tage.

$\rightarrow$ Die Übungshäufigkeit von mindestens einmal wöchentlich ist wirkungsvoll und am effektivsten. Die tägliche Übungsroutine erzielt bezüglich aktueller Symptombelastung und veränderten Entspannungserlebens keinen zusätzlichen Benefit. Die monatliche An- 
wendung vermag ein verändertes Entspannungserleben zu entwickeln, genügt jedoch nicht, um bestehende Beschwerden signifikant zu verbessern.

$\rightarrow$ Die regelmäßige Übungsroutine ist vom aktuellen Wohlbefinden und Beschwerdebild abhängig und wird vermehrt in Zeiten geringer Symptombelastung umgesetzt. Die Ergebnisse zeigen, dass besonders in Phasen des Wohlbefindens geübt wird. Je größer die Belastung durch anspannungsbezogene Beschwerden, desto unwahrscheinlicher ist ein erfolgreicher Alltagstransfer. Zum einen spricht dies für Nachsorgeprogramme zur Stabilisierung nach der Reha. Zum anderen ist dies ein Diskussionsansatz zukünftiger Kurse. Wie bereits in Kapitel 5.2 angesprochen, können sich die kurativen Wirkungen der Progressiven Relaxation erst entfalten, wenn auch in Zeiten vermehrter Symptombelastung geübt und von den Effekten profitiert wird. Der „eingebaute Tranquilizer“ (Jacobson, 2006) sollte gerade in solchen Phasen zum Einsatz kommen. Zukünftige Kursteilnehmer sollten darauf hingewiesen und zur Anwendung auch im hektischen Alltag motiviert werden. 


\subsection{Grenzen der Studie}

Die Evaluation des modifizierten, standardisierten Entspannungskurses im Vergleich zur ursprünglichen Umsetzung (Arndt, 2006) und die Verbesserung des Alltagstransfers von Progressiver Relaxation mittels Identifizierung möglicher Prädiktoren waren Ziele der vorliegenden Arbeit. Die Kursteilnehmer wurden regelmäßig angewiesen, die entsprechenden Fragebögen jeweils nur im Hinblick auf diesen Entspannungskurs auszufüllen. Zudem wurden statt allgemeiner Symptomchecklisten Messinstrumente eingesetzt, die speziell anspannungsbezogene Beschwerden erfassen. Um noch spezifischer die Auswirkungen der Entspannungsmethode zu erfassen, wurden die Veränderungen und Symptome nicht nur bezogen auf das gesamte Patientenkollektiv, sondern auch im Vergleich „(extrem) Übende“ vs. „(extrem) Nicht-Übende“ erfasst. Nichtsdestotrotz bleibt die Möglichkeit des Konfusionseffekts mit anderen Therapiemodulen bestehen. Die Veränderungen bestehender Beschwerden sowie des Entspannungserlebens und Wohlbefindens können zudem durch andere Bausteine der psychosomatischen Behandlung oder individuelle, private oder familiäre Faktoren bedingt und beeinflusst sein. Um diesen Effekt zu minimieren, müsste eine Kontrollgruppe ohne Progressive Relaxation dem Entspannungskurs gegenübergestellt werden. Eine solche kontrollierte Studie impliziert jedoch die Benachteiligung der Patienten der Kontrollgruppe, denen eine (positive) Erfahrung mit dieser nachgewiesen wirksamen Entspannungsmethode vorenthalten würde.

Bei der vorliegenden Stichprobe handelt es sich um ein Patientenkollektiv der Fachklinik für Psychosomatische Medizin und Psychotherapie in Blieskastel. 73,7\% waren weiblichen Geschlechts, das Alter lag im Mittel bei 49,14 Jahren ( $\mathrm{SD}=8,48)$. Weniger als $15 \%$ der Studienteilnehmer waren jünger als 40 Jahre. Bei 41,1\% der Patienten lag eine Anpassungsstörung, bei 13,9\% eine Angststörung vor. 33,1\% der Kursteilnehmer litten unter Depressionen, 11,9\% unter einer Schmerzstörung. 51 der eingeschlossenen Patienten konnten keiner dieser vier Hauptdiagnosen zugeordnet werden (Welsch, 2010). Zudem bestanden oftmals Komorbiditäten, die eine solche Zuordnung nur bedingt zuließen. Zusammenfassend bestand das eingeschlossene Patientenkollektiv zu einem Großteil aus Frauen in der zweiten Lebenshälfte mit Erkrankungen aus dem Formenkreis der affektiven- oder Anpassungsstörungen. Desweiteren wurden nur stationär aufgenommene Patienten eingeschlossen - Patienten, die im Rahmen von ambulanten Behandlungsmodulen oder Nachsorgeprogrammen an den Entspannungskursen teilnahmen, wurden nicht berücksichtigt. Die Evaluation von Entspannungskursen im ambulanten Bereich oder im Rahmen eines orthopädischen oder inter- 
nistischen Rehabilitationsaufenthalts und ein möglicher Vergleich mit der vorliegenden Studie wäre eine interessante Aufgabenstellung zukünftiger Forschungsarbeiten. Eine Studie mit jungen, berufstätigen Probanden könnte zudem aufschlussreiche Ergebnisse liefern.

Die Repräsentativität und der Umfang der Stichprobe konnten im Vergleich zur Pilotstudie verbessert und eine Drop-out-Analyse ermöglicht werden. Die Drop-out-Quote war insgesamt niedrig (33,3\% von Beginn der Studie bis zur Katamnese nach drei Monaten). Zudem war trotz der umfangreichen postalischen Befragung nach drei Monaten eine Rücklaufquote von 78,3\% erzielt worden. Darüber hinaus wurden die Fragebögen meist gänzlich ausgefüllt, sodass die Anzahl fehlender Items vernachlässigbar war. Die Compliance der Studienteilnehmer dieser Arbeit war im Gegensatz zur Pilotstudie somit zufriedenstellend. Das von Ice bereits im Jahre 1985 geforderte kreative, experimentelle und innovative Verhalten und Programmdesign zur Verbesserung der Compliance wurde umgesetzt, die von Vogler et al. (1982) betonte ungestörte Beziehung zwischen Kursleiter und Kursteilnehmer konnte aufgebaut werden: Das Erreichen dieses umfassenden Datensatzes war nämlich nur durch kontinuierlichen Einsatz der beteiligten Personen möglich. Regelmäßiger persönlicher Kontakt zu den Kursteilnehmern, das Anbieten weiterer Kursstunden an Feiertagen sowie die individuelle Betreuung bei Fragen ermöglichten die entsprechenden Bedingungen einer möglichst umfassenden Datenerhebung. Das Antwortverhalten nach dem Phänomen der sozialen Erwünschtheit (Möhring und Schlütz, 2010) darf jedoch gerade aufgrund dieses vermehrten persönlichen Kontakts zwischen Kursleitern und Kursteilnehmern nicht unterschätzt werden. Die Bewertung der persönlichen Beschwerden, des veränderten Entspannungserlebens, des Entspannungskurses als auch die Angaben hinsichtlich Übungshäufigkeit während des Reha-Aufenthalts und im Alltag müssen vor diesem Hintergrund kritisch betrachtet werden. Nichtsdestotrotz bleibt diese Einschränkung als Preis eines umfassenden Datensatzes mit geringer Dropout-Quote tragbar. 


\subsection{Ausblick}

Die nachhaltige Wirksamkeit der Progressiven Relaxation konnte durch die vorliegende Studie abermals bestätigt werden. Die Evaluation des modifizierten Entspannungskurses nach standardisiertem Konzept zeigte deutliche Verbesserungen. Zudem wurden Prädiktoren identifiziert, die Ansatzpunkte zukünftiger Kurse sein können, um einen Alltagstransfer dieser Entspannungsmethode zu unterstützen.

Im Rahmen der vorliegenden Studie wurde jedoch lediglich ein geringer Teil des umfassenden Datensatzes ausgewertet. Das verbleibende Datenmaterial bietet zahlreiche Ansatzpunkte für zukünftige Fragestellungen. So wurden beispielsweise die Protokollbögen nach jeder Kursstunde nicht berücksichtigt. Eine Auswertung dieser Fragebögen könnte weitere Informationen zur Prozessevaluation liefern. Spannend wären die Fragen, inwiefern sich die Entspannung der Muskelgruppen im Verlauf des Kurses entwickelt, wann und in welcher Stunde vielleicht spezifische Probleme auftreten und welchen Einfluss die übrigen Teilnehmer, die Dauer der Kursstunden oder die Phantasiereisen auf den Entspannungszustand und deren Entwicklung haben.

Studien im ambulanten Bereich oder innerhalb anderer Fachrichtungen mit jungem oder jugendlichem Patientenkollektiv könnten interessante Ergebnisse zur Wirkung der Progressiven Relaxation bei unterschiedlichen Altersgruppen liefern.

Darüber hinaus sollte dem Wunsch der Patienten nach begleitendem Kursmaterial in Form einer CD Folge geleistet werden. Die Aufnahme der angewandten Übungstexte auf CD, gesprochen von den entsprechenden Kursleitern, war oftmals expliziter Verbesserungsvorschlag verschiedenster Kursteilnehmer - während und nach Abschluss des Kurses sowie abermals im Rahmen der katamnestischen Befragung nach drei Monaten. Eine Nachfolgestudie könnte den kurz- und langfristigen Einfluss solcher auditorischer Hilfsmittel sowie die Bedeutung des Themas „Stimmfixierung“ (z. B. Männer- vs. Frauenstimme) untersuchen und einen Vergleich zur vorliegenden Arbeit ziehen. Möglicherweise könnte durch diesen Einsatz ein weiterer wichtiger Beitrag zur Verbesserung des Alltagstransfers von Progressiver Relaxation geleistet werden. 


\section{Literaturverzeichnis}

1. Arndt M (Erhebungszeitraum 2005/2006, vorgelegt 2007) Transfer von Entspannungsverfahren aus der stationären Rehabilitation in den Alltag der Patienten. Dissertation zur Erlangung des Grades eines Doktors der Medizin der Medizinischen Fakultät der Universität des Saarlandes

2. Baider L, Uziely B, De-Nour AK (1994) Progressive muscle relaxation and guided imagery in cancer patients. General Hospital Psychiatry 16: 340-347

3. Baird CL, Sands L (2004) A pilot study of the effectiveness of guided imagery with progressive muscle relaxation to reduce chronic pain and mobility difficulties of osteoarthritis. Pain Management Nursing 5: 97-104

4. Bandura A (1977) Self-efficacy: Toward a unifying theory of behavioral change. Psychological Review 84: 191-215

5. Basmajian JV (1963) Control and training of single motor units. Science 141: 440-441

6. Benson H (1975) The Relaxation Response. William Morrow, New York

7. Bernardy K, Krampen G, Köllner V (2008) Prädiktoren des Alltagstransfers eines stationär erlernten Entspannungstrainings. Die Rehabilitation 47: 359-365

8. Bernheim H (1891) Hypnotism, Suggestion, Psychotherapie. Etudes Nouvelles. Doin Paris

9. Bernheim H (1892) Neue Studien über Hypnotismus, Suggestion und Psychotherapie. Deuticke, Leipzig Wien

10. Bernstein DA, Borkovec TD (1973) Progressive Relaxation Training: A manual for the helping professions. Research Press Company, Champaign Illinois 
11. Bernstein DA, Borkovec TD $(1975,2002)$ Entspannungstraining. Handbuch der progressiven Muskelentspannung. Pfeiffer bei Klett-Cotta, Stuttgart

12. Betts GH (1909) The distribution and functions of mental imagery. Columbia University Teachers College. Contribution to Education Series 26, New York

13. Blanchard EB, Andrasik F, Neff DF, Arena JG, Ahlex TA, Jurish SE, Pallmeyer TP, Saunders NL, Teders SJ, Barron KD, Rodichok LD (1982) Biofeedback and relaxation training with three kinds of headache: Treatment effects and their prediction. Journal of Consulting and Clinical Psychology 50: 562-575

14. Blanchard EB, Appelbaum KA, Radnitz CL, Michultka D, Morrill B, Kirsch C, Hillhouse J, Evans DD; Guarnieri P, Attanasio V, Andrasik F, Jaccard J, Dentinger MP (1990) Placebo-controlled evaluation of abbreviated progressive muscle relaxation combined with cognitive therapy in the treatment of tension headache. Journal of Consulting and Clinical Psychology 58: 210-215

15. Borkovec TD, Fowles DC (1973) Controlled investigation of the effects of progressive and hypnotic relaxation on insomnia. Journal of Abnormal Psychology 82: 153-158

16. Borkovec TD, Sides JK (1979) Critical procedural variables to the physiological effects of progressive relaxation: A review. Behaviour Research and Therapy 17: 119-125

17. Braid, J (1846) Die Macht des Geistes über den Körper. Eine experimentelle Untersuchung. In Braid J (ed) (1882) Der Hypnotismus. Ausgewählte Schriften. Paetel, Berlin

18. Brauer AP, Horlick L, Nelson E, Farquhar JW, Agras WS (1979) Relaxation therapy for essential hypertension: A Veterans Administration outpatient study. Journal of Behavioral Medicine 2: 21-29

19. Brenner H (2002) Progressives Entspannungstraining. Pabst, Lengerich

20. Brenner H (2004) Meditation. Die wichtigsten Methoden, Ziele und Übungen. Humboldt Verlag, Baden Baden 
21. Bundesausschuss der Ärzte und Krankenkassen (1998) Richtlinien des Bundesausschusses der Ärzte und Krankenkassen über die Durchführung der Psychotherapie (Psychotherapie-Richtlinien) in der Fassung vom 11.Dezember 1998. Bundesanzeiger 1999, 6: 249

22. Cahn BR, Polich J (2006) Meditation states and traits: EEG, ERP, and neuroimaging studies. Psychological Bulletin 132: 180-211

23. Cannon WB (1914) The emergency function of the adrenal medulla in pain and major emotions. American Journal of Physiology 33: 356-372

24. Carlson CR, Hoyle RH (1993) Efficacy of abbreviated progressive muscle relaxation training: A quantitative review of Behavioral Medicine Research. Journal of Consulting and Clinical Psychology 61: 1059-1067

25. Charcot JM (1892-94) Poliklinische Vorträge (Bd. 1 übers. von S. Freud). Deuticke, Wien

26. Chen WC, Chu H, Lu RB, Chou YH, Chen $\mathrm{CH}$, Chang YC, O`Brien AP, Chou KR (2009) Efficacy of progressive muscle relaxation training in reducing anxiety in patients with acute schizophrenia. Journal of Clinical Nursing 18: 2187-2196

27. Cohen J (1988) Statistical Power Analysis for the Behavioral Sciences. 2nd ed. Lawrence Erlbaum Associates, Hillsdale

28. Davidson RJ, Kabat-Zinn J, Schumacher J, Rosenkrantz M, Muller D, Santorelli SF, Urbanowski F, Harrington A, Bonus K, Sheridan JF (2003) Alterations in brain and immune function produced by mindfulness meditation. Psychosomatic Medicine 65: 564570

29. Davis M, Eshelman ER, McKay M (2008) The Relaxation and Stress Reduction Workbook. New Harbinger Publications, Oakland 
30. Demiralp M, Oflaz F, Komurcu S (2010) Effects of relaxation training on sleep quality and fatigue in patients with breast cancer undergoing adjuvant chemotherapy. Journal of Clinical Nursing 19: 1073-1083

31. Derra C (2006) Stressbewältigung - ein Eckbaustein der Gesundheitsbildung in heutiger Zeit. In Pusch HJ, Biendarra I (eds) (2006) Gesundheitsbildung im Lebenslauf. Königshausen und Neumann, Würzburg, pp 67-78

32. Derra C (2007) Progressive Relaxation. Grundlagen und Praxis für Ärzte und Therapeuten. Deutscher Ärzte Verlag, Köln

33. Deutsche Rentenversicherung Bund. http://www.deutsche-rentenversicherung-bund.de/ SharedDocs/de/Navigation/Service/Zielgruppen/Sozialmedizin_Forschung/qualitaetssich erung/reha_therapiestandards_node.html (08.01.2011)

34. Diehl BJM (1987) Autogenes Training und gestufte Aktivhypnose. Psychophysiologische Aspekte. Springer, Berlin Heidelberg New York

35. Dunlap WP, Cortina JM, Vaslow JB, Burke MJ (1996) Meta-analysis of experiments with matched groups or repeated measures design. Psychological methods 1: 170-17

36. Eller LS (1999) Effects of cognitive-behavioral interventions on quality of life in persons with HIV. International Journal of Nursing Studies 36: 223-233

37. Emery CF, France CR, Harris J, Norman G, Van Arsdalen C (2008) Effects of progressive muscle relaxation training on nociceptive flexion reflex threshold in healthy young adults: A randomized trial. Pain 138: 375-379

38. Freyth C, Elsesser K, Lohrmann T, Sartory G (2010) Effects of additional prolonged exposure to psychoeducation and relaxation in acute stress disorder. Journal of Anxiety Disorders 24: 909-917

39. Fried RG, Hussain SH (2008) Nonpharmacologic management of common skin and psychocutaneous disorders. Dermatologic Therapy 21: 60-68 
40. Friederich M, Trippe RH, Özcan M, Weiss T, Hecht H, Miltner WH (2001) Laser-evoked potentials to noxious stimulation during hypnotic analgesia and distraction of attention suggest different brain mechanisms of pain control. Psychophysiology 38: 768-776

41. Goldfried MR (1971) Systematic desensitization as training in self-control. Journal of Consulting and Clinical Psychology 37: 228-234

42. Grawe K, Donati R, Bernauer F (1994) Psychotherapie im Wandel. Von der Konfession zur Profession. Hogrefe, Göttingen

43. Grover N, Kumaraiah V, Prasadrao PS, D’souza G (2002) Cognitive behavioral intervention in bronchial asthma. Journal of Association of Physicians of India 50: 896-900

44. Hamm A (2009) Progressive Muskelentspannung. In Petermann F, Vaitl D (eds) (2009) Entspannungsverfahren - Das Praxishandbuch. 4. Auflage. Beltz Verlag, Weinheim Basel, pp 143-164

45. Han KS (2002) The effect of an integrated stress management program on the psychologic and physiologic stress reactions of peptic ulcer in Korea. International Journal of Nursing Studies 39: 539-548

46. Heckhausen J, Heckhausen H (2006) Motivation und Handeln. Springer Medizin Verlag, Heidelberg

47. Hillenberg JB, Collins FL Jr. (1983) The importance of home practice for progressive relaxation training. Behaviour Research and Therapy 21: 633-642

48. Hoelscher TJ (1987) Maintenance of relaxation-induced blood pressure reductions: the importance of continued relaxation practice. Biofeedback and Self Regulation 12: 3-12

49. Holland JC, Morrow GR, Schmale A, Derogatis L, Stefanek M, Berenson S, Carpenter PJ, Breitbart W, Feldstein M (1991) A randomized clinical trial of alprazolam versus progressive muscle relaxation in cancer patients with anxiety and depressive symptoms. Journal of Clinical Oncology 9: 1004-1011 
50. Hölzel BK, Ott U, Hempel H, Hackl A, Wolf K, Stark R, Vaitl D (2007) Differential engagement of anterior cingulate and adjacent medial frontal cortex in adept mediators and non-mediators. Neuroscience Letters 421: 16-21

51. Hölzel BK, Ott U, Gard T, Hempel H, Weygandt M, Morgen K, Vaitl D (2008) Investigation of mindfulness meditation practitioners with voxel-based morphometry. Social Cognitive and Affective Neuroscience 3: 55-61

52. Hui PN, Wan M, Chan WK, Yung PM (2006) An evaluation of two behavioral rehabilitation programs, qigong versus progressive relaxation, in improving the quality of life in cardiac patients. The journal of alternative and complementary medicine 12: 373-378

53. Huntley A, White AR, Ernst E (2002) Relaxation therapies for asthma: A systematic review. Thorax 57: 127-131

54. Hyman RB, Feldman HR, Harris RB, Levin RF, Malloy GB (1989) The effects of relaxation training on clinical symptoms: A Meta-Analysis. Nursing Research 38: 216220

55. Ice R (1985) Long-term compliance. Psychical therapy 65: 1832-1839

56. Israel L, Rohmer F, Geissmann P (1958) Variations électroencéphalographiques au cours de la relaxation autogèneet de l'hypnose. In Aboulker P, Chertok L, Sapir M (eds) La relaxation. Aspects théoretiques et practiques. Expansion Scientifique Francaise, Paris

57. Jacobson E (1929) Progressive Relaxation. A physiological and clinical investigation of muscular states and their significance in psychology and medical practice. The University of Chicago Press, Chicago Illinois

58. Jacobson E (1934) You must relax. A practical method of reducing the strains of modern living. Whittlesey House, McGraw-Hill, New York London

59. Jacobson E (1938a) Progressive relaxation. University of Chicago Press, Chicago 
60. Jacobson E (1938b) You can sleep well. Whittlesey House, McGraw-Hill, New York London

61. Jacobson E (1944) The peace we Americans need. A. Kroch and Son Publishers, Chicago

62. Jacobson E (1947) The influence of relaxation upon the blood pressure in "essential" hypertension. Federation Proceedings 6: 135-136

63. Jacobson E (1955) Immer mit der Ruhe - eine praktische Methode, die Anspannungen des modernen Lebens zu lindern. Gerd Hartje Verlag, Stuttgart

64. Jacobson E (1959) How to relax and have your baby. McGraw-Hill, New York London

65. Jacobson E (1963) Tension control for businessmen. McGraw-Hill, New York London

66. Jacobson E (1973) Teaching and learning, New methods for old arts. National Foundation for Progressive Relaxation, Chicago Illinois

67. Jacobson E (2006) Entspannung als Therapie. Progressive Relaxation in Theorie und Praxis. Klett-Cotta, Stuttgart

68. Janke J (1999) The effect of relaxation therapy on preterm labor outcomes. Journal of Obstetric, Gynecologic and Neonatal Nursing 28: 255-263

69. Janssen K, Neutgens J (1986) Autogenic training and progressive relaxation in the treatment of three kinds of headaches. Behaviour Research and Therapy 24: 199-208

70. Jevning R, Pirkle HC, Wilson AF (1977) Behavioral alteration of plasma phenylalanine concentration. Physiology and Behavior 19: 611-614

71. Jevning R, Wilson AF, Davidson JM (1978) Adrenocortical activity during meditation. Hormones and Behavior 10: 54-60 
72. Jevning R, Wilson AF, Vander Laan EF (1978) Plasma prolactin and growth hormone during meditation. Psychosomatic Medicine 40: 329-333

73. Jevning R, Wallace RK, Beidebach M (1992) The physiology of meditation: A review. A wakeful hypometabolic integrated response. Neuroscience and Behavioral Reviews 16: $415-424$

74. Jorm AF, Christensen H, Griffiths KM, Parslow RA, Rodgers B, Blewitt KA (2004) Effectiveness of complementary and self-help treatments for anxiety disorders. The Medical Journal of Australia 181: 29-46

75. Jorm AF, Morgan AJ, Hetrick SE (2008) Relaxation for depression. Cochrane Database of Systematic Reviews Issue 4

76. Keefe FJ, Surwit RS, Pilon RN (1980) Biofeedback, autogenic training, and progressive relaxation in the treatment of Raynaud's disease: A comparative study. Journal of Applied Behavior Analysis 13: 3-11

77. Kirn T, Eichelmeyer L, Engberding M (2009) Imagination in der Verhaltenstherapie. Springer Medizin Verlag, Heidelberg

78. Klinkenberg N (2008) Entspannungsverfahren. In: Schmid-Ott G, Wiegand-Grefe S, Jacobi C, Paar G, Meermann R, Lamprecht F (eds) (2008) Rehabilitation in der Psychosomatik. Versorgungsstrukturen, Behandlungsangebote, Qualitätsmanagement. Schattauer Verlag, Stuttgart New York, pp 197-200

79. Krampen G, Ohm D (1985) Clinical perception and recovery in myocardial infarct patients at a rehabilitation clinic. Rehabilitation 24: 64-68

80. Krampen G (1991) Diagnostisches und Evaluatives Instrumentarium zum Autogenen Training (AT-EVA). Hogrefe, Göttingen 
81. Krampen G (2002a) Entspannungstraining und -therapie: Anamnesebogen (ET-ANAM). Teil 1 des Diagnostischen und Evaluativen Instrumentariums für Entspannungstraining und Entspannungstherapie (ET-EVA). Manual. Hogrefe, Göttingen

82. Krampen G (2002b) Prognostischer Wert von Vorerfahrungen und Teilnahmemotiven für den Lern- und Transferprozess bei Autogenem Training und Progressiver Relaxation. Entspannungsverfahren 19: 5-24

83. Krampen G (2003) Entspannungstraining und -therapie: Änderungssensitive Symptomliste (ET-SYM). Hogrefe, Göttingen (in Vorbereitung)

84. Krampen G (2004) Differentielle Indikation von Autogenem Training und Progressiver Relaxation. Entspannungsverfahren 21: 6-27

85. Krampen G (2006) Änderungssensitive Symptomliste zu Entspannungserleben, Wohlbefinden, Beschwerden- und Problembelastung (ASS-SYM).Hogrefe, Göttingen

86. Krampen G, Eye A von (2006) Treatment motives as predictors of acquisition and transfer of relaxation methods to everyday life. Journal of Clinical Psychology 62: 83-96

87. Kwekkeboom KL, Gretarsdottir E (2006) Systematic review of relaxation interventions for pain. Journal of Nursing Scholarship 38: 269-277

88. Kwekkeboom KL, Hau H, Wanta B, Bumpus M (2008) Patients` perception of the effectiveness of guided imagery and progressive muscle relaxation interventions used for cancer pain. Complementary Therapies in Clinical Practice 14: 185-194

89. Lammers CA, Naliboff BD, Straatmeyer AJ (1984) The effects of progressive relaxation on stress and diabetic control. Behaviour Research and Therapy 22: 641-650

90. Lazarus RS; Launier R (1981) Stressbezogene Transaktionen zwischen Person und Umwelt. In Nitsch JR (ed) Stress, Theorien, Untersuchungen, Maßnahmen. Huber, Bern, pp 213-259 
91. Lehrer PM, Woolfolk RL (1984) Are stress reduction techniques interchangeable, or do they have specific effects? A review of comparative empirical literature. In Woolfolk RL, Lehrer PM (eds) Principles and practice of stress management. Guilford Press, New York, pp 404-477

92. Lempa M, Grotemeyer KH, Häuser W, Moormann O, Wesselmann U, Derra C (2004) Diagnostik und Therapie funktioneller Schmerzsyndrome. Schmerz 18: 141-144

93. Lichstein KL (1988) Clinical relaxation strategies. John Wiley and Sons, New York

94. Linden W (2000) Meditation. In Vaitl D, Petermann F (2000) Handbuch der Entspannungsverfahren. Psychologie Verlags Union, Weinheim, pp 256-268

95. Linton SJ, Götestam KG (1984) A controlled study of the effects of applied relaxation plus operant procedures in the regulation of chronic pain. British Journal of Clinical Psychology 23: 291-299

96. Luthe W (ed) (1969/1970) Autogenic therapy. Vol. I-IV. Grune and Stratton, New York

97. Maharishi MY (1975) Transcendental Meditation. Plume, New York

98. Maierhofer A, Schmied W, Köllner V (2008) Progressive Muskelentspannung. Manual für Therapeuten. MediClin Bliestal Kliniken, Blieskastel

99. Manzoni GM, Pagnini F, Castelnuovo G, Molinari E (2008) Relaxation training for anxiety: a ten-year systematic review with meta-analysis. BMC Psychiatry 8: 41

100.McGlynn FD, Moore PM, Lawyer S, Karg R (1999) Relaxation training inhibits fear and arousal during in vivo exposure to phobia-cue stimuli. Journal of Behavior Therapy and Experimental Psychiatry 30: 155-168

101.Means MK, Lichstein KL, Epperson MT, Johnson CT (2000) Relaxation therapy for insomnia: nighttime and day time effects. Behaviour Research and Therapy 38: 665-678 
102.Mitchell KR, Mitchell DM (1971) Migraine: An exploratory treatment application of programmed behavior therapy techniques. Journal of Psychosomatic Research 15: 137157

103.Möhring W, Schlütz D (2010) Die Befragung in der Medien- und Kommunikationswissenschaft. Eine praxisorientierte Einführung. 2. Auflage. VS Verlag für Sozialwissenschaften, Wiesbaden

104.Molassiotis A (2000) A pilot study of the use of progressive muscle relaxation training in the management of post-chemotherapy nausea and vomiting. European Journal of Cancer Care 9: 230-234

105.Molassiotis A, Yung HP, Yam BM, Chan FY, Mok TS (2002) The effectiveness of progressive muscle relaxation training in managing chemotherapy-induced nausea and vomiting in Chinese breast cancer patients: A randomized controlled trial. Support Care Cancer 10: 237-246

106.Möller HJ, Laux G. Kapfhammer HP (2005) Psychiatrie und Psychotherapie. 2. Auflage. Springer Medizin Verlag. Heidelberg

107.Murphy AI, Lehrer PM, Jurish S (1990) Cognitive skills training and relaxation training as treatments for tension headaches. Behavior Therapy 21: 89-98

108.Nickel C, Kettler C, Muehlbacher M, Lahmann C, Tritt K, Fartacek R, Bachler E, Rother N, Egger C, Rother WK, Loew TH, Nickel MK (2005) Effect of progressive muscle relaxation in adolescent female bronchial asthma patients: A randomized, double-blind, controlled study. Journal of Psychosomatic Research 59: 393-398

109.Nickel C, Lahmann C, Muehlbacher M, Pedrosa Gil F, Kaplan P, Buschmann W, Tritt K, Kettler C, Bachler E, Egger C, Anvar J, Fartacek R, Loew T, Rother W, Nickel M (2006) Pregnant women with bronchial asthma benefit from progressive muscle relaxation: A randomized, prospective, controlled trial. Psychotherapy and Psychosomatics 75: 237243 
110.Öst LG (1987) Applied relaxation: description of a coping technique and review of controlled studies. Behaviour Research and Therapy 25: 397-407

111.Ohm D (2004) Bisherige Ergebnisse der Konsensuskonferenzen zur Progressiven Relaxation. Entspannungsverfahren 21: 83-89

112.Ohm D (2007) Stressfrei durch Progressive Relaxation. Mehr Gelassenheit durch Tiefenmuskelentspannung nach Jacobson. TRIAS Verlag, Stuttgart

113.Olschewski A (1996) Progressive Muskelentspannung - Stressbewältigung und Gesundheitsprävention mit klassischen und neuen Übungen nach Jacobson. Haug, Heidelberg

114.Ott U (2009) Meditation. In Petermann F, Vaitl D (2009) Entspannungsverfahren - Das Praxishandbuch. 4. Auflage. Beltz Verlag, Weinheim Basel, pp 132-142

115.Paar GH, Grohmann S (2008) Stationäre psychosomatische Rehabilitation. In Schmid-Ott G, Wiegand-Grefe S, Jacobi C, Paar G, Meermann R, Lamprecht F (eds) (2008) Rehabilitation in der Psychosomatik. Versorgungsstrukturen, Behandlungsangebote, Qualitätsmanagement. Schattauer Verlag, Stuttgart New York, pp 55-74

116.Paar GH, Wiegand-Grefe S (2001) Störungsspezifische Psychotherapie in der Psychosomatischen Rehabilitation. In: Bassler M (ed) Störungsspezifische Ansätze in der stationären Psychotherapie. Psychosozial-Verlag, Gießen, pp 176-204

117.Paul GL (1969) Physiological effects of relaxation training and hypnotic suggestion. Journal of Abnormal Psychology 74: 425-437

118.Paul GL, Trimble RW (1970) Recorded versus live relaxation and hypnotic suggestion: comparative effectiveness for reducing physiological arousal and inhibiting stress responses. Behavior Therapy 1: 285-302

119.Pawlow LA, Jones GE (2005) The impact of abbreviated progressive muscle relaxation on salivary cortisol and salivary immunglobulin A (sIgA). Applied Psychophysiology and Biofeedback 30: 375-387 
120.Payne RA (1998) Entspannungstechniken. Gustav Fischer, Lübeck Stuttgart Jena Ulm

121.Petermann F, Vaitl D (2009) Entspannungsverfahren - Das Praxishandbuch. 4.Auflage. Beltz Verlag, Weinheim Basel

122.Philips C, Hunter M (1981) The treatment of tension headache-II.EMG "normality" and relaxation. Behaviour Research and Therapy 19: 499-507

123.Rainforth MV, Schneider RH, Nidich SI, Gaylord-King C, Salerno JW, Anderson JW (2007) Stress reduction programs in patients with elevated blood pressure: A systematic review and meta-analysis. Current Hypertension Reports 9: 520-528

124.Reusch A, Ströbl V (2004) Motivation zur Entspannungsübung bei Rehabilitanden mit Erkrankungen der Bewegungsorgane. Aktuelle Rheumatologie 29: 75-82

125.Ruhl U, Hach I, Wittchen HU (2006) Entspannungsverfahren. In Wittchen HU, Hoyer J Klinische Psychologie und Psychotherapie. Springer Medizin Verlag, Heidelberg

126. Schacter DL (1977) EEG theta waves and psychological phenomena: A review and analysis. Biological Psychology 5: 47-82

127.Schmid-Ott G, Wiegand-Grefe S, Jacobi C, Paar G, Meermann R, Lamprecht F (eds) (2008) Rehabilitation in der Psychosomatik. Versorgungsstrukturen, Behandlungsangebote, Qualitätsmanagement. Schattauer Verlag, Stuttgart New York

128. Schultz JH (1924) Suggestion und Hypnose. Niels Kampmann

129. Schultz JH (1934) Der Yoga und die deutsche Seele. Allgemeines ärztliches Zentralblatt für Psychotherapie 7: 61-69

130.Schultz JH (1956) Das autogene Training (Konzentrative Selbstentspannung). 9. Auflage. Thieme, Stuttgart

131.Schultz JH (1969) Übungsheft für das Autogene Training. 14. Auflage. Thieme, Stuttgart 
132.Schultz JH (1970) Das Autogene Training. 13. Auflage. Thieme, Stuttgart

133.Schultz JH (1973) Das Autogene Training, Konzentrative Selbstentspannung. 14. Auflage. Thieme, Stuttgart

134.Schwartz FW, Badura B, Busse R, Leidl R, Raspe H, Siegrist J, Walter U (eds) (2003) Das Public Health Buch. Gesundheit und Gesundheitswesen. Urban und Fischer, München Jena

135.Schwartz M, Andrasik F (2005) Biofeedback. A practitioner`s guide. 3nd ed. Guilford, New York

136. Seyle H (1952) The story of the adaptation syndrome. Acta, Montreal

137.Seyle H (1978) The Stress of Life. Time Life Books, New York

138. Seyle H (1984) Stress, mein Leben, Erinnerungen eines Forschers. Fischer, Frankfurt/ Main

139. Shapiro JR, Pisetsky EM, Crenshaw W, Spainhour S, Hamer RM, Dymek-Valentine M, Bulik CM (2008) Exploratory study to decrease postprandial anxiety: Just relax! International Journal of Eating Disorders 41: 728-733

140.Sheu S, Irvin BL, Lin HS, Mar CL (2003) Effects of progressive muscle relaxation on blood pressure and psychosocial status for clients with essential hypertension in Taiwan. Holistic Nursing Practice 17: 41-47

141.Singh VP, Rao V, Prem V, Sahoo RC, KeshavPai K (2009) Comparison of the effectiveness of music and progressive muscle relaxation for anxiety in COPD - A randomized controlled pilot study. Chronic Respiratory Disease 6: 209-216

142.Smith JS (1988) Step toward a cognitive-behavioral model of relaxation. Biofeedback and Self-Regulation 13: 307-329 
143.Southam MA; Agras WS, Taylor CB, Kraemer HC (1982) Relaxation training: Blood pressure lowering during the working day. Achives of General Psychiatry 39: 715-717

144.Stetter F (2004) Entspannungsverfahren - Wirksame Komponenten psychotherapeutischer und psychiatrischer Behandlung. Psychotherapeut 49: 281-291

145.Stiles M (2003) Structural Yoga Therapy. Weiser Books, Boston

146.Ströbl V, Reusch A, Ellgring H (2004) Konstruktion eines Verfahrens zur Erfassung der Motivation zu Entspannungsübungen. Zeitschrift für Gesundheitspsychologie 12: 65-74

147.Taylor DN (1995) Effects of a behavioral stress-management program on anxiety, mood, self-esteem, and T-cell count in HIV positive men. Psychological Reports 76: 451-457

148. Tindle HA, Barbeau EM, Davis RB, Eisenberg DM, Park ER, Phillips RS, Rigotti NA (2006) Guided imagery for smoking cessation in adults: A randomized pilot trail. Complementary Health Practice Review 11: 166-175

149. Turner JA (1982) Comparison of group progressive-relaxation training and cognitivebehavioural therapy for chronic low back pain. Journal of Consulting and Clinical Psychology 50: 757-765

150.Turner JA, Chapman CR (1982a) Psychological Interventions for chronic pain: A critical review. I. Relaxation training and biofeedback. Pain 12: 1-21

151.Turner JA, Chapman CR (1982b) Psychological Interventions for chronic pain: A critical review. II. Operant conditioning, hypnosis, and cognitive-behavioral therapy. Pain 12: 23-46

152. Vaitl D, Petermann F (2000) Handbuch der Entspannungsverfahren. Psychologie Verlags Union, Weinheim

153. Vaitl D, Petermann F (2004) Entspannungsverfahren - Das Praxishandbuch. Psychologie Verlags Union, Weinheim 
154.Van Dixhoorn J, White A (2005) Relaxation therapy for rehabilitation and prevention in ischaemic heart disease: A systematic review and meta-analysis. European Journal of Cardiovascular Prevention and Rehabilitation 12: 193-202

155.Verband Deutscher Rentenversicherungsträger (VDR) (2007) Rahmenkonzept zur medizinischen Rehabilitation in der gesetzlichen Rentenversicherung. Deutsche Rentenversicherung Bund, Berlin

156. Vogler WD, Westphal ML, Trautmann E, Geyer M (1982) Motive for premature terminations of AT group treatment. Psychiatrie, Neurologie und medizinische Psychologie 34: 295-300

157.Wallace RK (1970) Physiological effects of transcendental meditation. Science 167: $1751-1754$

158.Walter U, Bramesfeld A, Plaumann M (2008) Prävention und Gesundheitsförderung. In Schmid-Ott G, Wiegand-Grefe S, Jacobi C, Paar G, Meermann R, Lamprecht F (eds) (2008) Rehabilitation in der Psychosomatik. Versorgungsstrukturen, Behandlungsangebote, Qualitätsmanagement. Schattauer Verlag, Stuttgart New York, pp 21-26

159.Weber S (1996) The effects of relaxation exercises on anxiety levels in psychiatric inpatients. Journal of Holistic Nursing 14: 196-205

160.Welsch K (2010) Ist Progressive Relaxation als „Einheitsentspannung“ noch zeitgemäß? Zur differentiellen Effektivität der Progressiven Relaxation in der psychosomatischen Rehabilitation in Abhängigkeit von Störungsbildern. Diplomarbeit in der Fachrichtung Psychologie der Universität des Saarlandes

161.Wiegand-Grefe S, Mans E (2008) Der Bezugstherapeut in der psychosomatischen Rehabilitation. In: Schmid-Ott G, Wiegand-Grefe S, Jacobi C, Paar G, Meermann R, Lamprecht F (eds) (2008) Rehabilitation in der Psychosomatik. Versorgungsstrukturen, Behandlungsangebote, Qualitätsmanagement. Schattauer Verlag, Stuttgart New York, pp 165-175 
162.Wilk C, Turkoski B (2001). Progressive muscle relaxation in cardiac rehabilitation: A pilot study. Rehabilitation Nursing 26: 238-243

163.Wolpe J (1958) Psychotherapy by reciprocal inhibition. Stanford University Press, Stanford CA

164. Yoo HJ, Ahn SH, Kim SB, Kim WK; Han OS (2005) Efficacy of progressive muscle relaxation training and guided imagery in reducing chemotherapy side effects in patients with breast cancer and in improving their quality of life. Support Cancer13: 826-833

165.Yu DS, Lee DT, Woo J (2007a) Effects of relaxation therapy on psychological distress and symptom status in older Chinese patients with heart failure. Journal of Psychosomatic Research 62: 427-437

166.Yu DS, Lee DT, Woo J,Hui E (2007b) Non-pharmacological interventions in older people with heart failure: effects of exercise training and relaxation therapy. Gerontology 53: $74-81$

167.Yu DS, Lee DT, Woo J (2010) Improving health-related quality of life of patients with chronic heart failure: effects of relaxation therapy. Journal of Advanced Nursing 66: 392403 


\section{Publikationen}

1. Klosterhalfen S, Becker N, Welsch K, Köllner V (2010) Prädiktoren des Alltagstransfers von Progressiver Muskelrelaxation nach stationärer psychosomatischer Rehabilitation. Abstractband zum 19. Rehabilitationswissenschaftlichen Kolloquium. DRV-SchriftenBand 88: 489-490

2. Welsch K, Becker N, Klosterhalfen S, Kerkhoff G, Köllner V (2010) Differentielle Effektivität Progressiver Muskelrelaxation nach Jacobson in der psychosomatischen Rehabilitation in Abhängigkeit von Störungsbildern. Abstractband zum 19. Rehabilitationswissenschaftlichen Kolloquium. DRV-Schriften-Band 88: 495-496

3. Klosterhalfen S, Becker N, Welsch K, Köllner V (2010) Vorhersage des Alltagstransfers von Progressiver Muskelrelaxation nach einer stationären psychosomatischen Rehabilitation. Psychologische Medizin Sondernummer 2010: 101-102

4. Welsch K, Becker N, Klosterhalfen S, Kerkhoff G, Köllner V (2010) Ist Progressive Muskelrelaxation nach Jacobson als „Einheitsentspannung“ in der psychosomatischen Rehabilitation noch zeitgemäß? Psychologische Medizin Sondernummer 2010: 100

5. Klosterhalfen S, Becker N, Welsch K, Köllner V (2010) Prediction of the transfer into daily life of progressive relaxation after an inpatient psychosomatic rehabilitation. Journal of Psychosomatic Research 68: 637-638

Zur Publikation in "Die Rehabilitation" eingereicht:

Klosterhalfen S, Welsch K, Becker N, Köllner V. Verbesserung des Transfers von Progressiver Relaxation in den Alltag der Patienten nach stationärer psychosomatischer Rehabilitation 


\section{Dank}

Mein besonderer Dank gilt Herrn Prof. Volker Köllner, der mich während der Zeit der Entstehung dieser Arbeit gut betreute, mit Rat und Tat zur Seite stand und mir gerade auch durch die Arbeit in seiner Klinik die Möglichkeit gab, „nah am Geschehen zu sein“ und gleichzeitig einen Einblick in diesen Fachbereich zu bekommen.

Ich danke Katja Welsch (und unserm Säbelzahntiger) - denn ohne sie hätten die Entspannungskurse, Kongresse und viele Stunden in Blieskastel und Saarbrücken nur halb so viel Spaß gemacht! Vielen Dank an Dr. Nicolas Becker für sein ,immer offenes Emailpostfach“ und seine Hilfe bei der statistischen Auswertung!

Vielen lieben Dank auch an Lena Gabriel für ihre Hilfe bei der englischen Übersetzung und Monika Heinz, die sich trotz „besonderer Umstände“ Zeit für meine Arbeit genommen hat! Markus Uhl und Elisabeth Krämer danke ich für die Hilfe bei der äußeren Form! Vielen lieben Dank an Philipp Kulas für sein ,wachsames Auge“ und seine Unterstützung!

Besonderer Dank gebührt meiner Mutter für das immer gewissenhafte Korrekturlesen und ihren Rückhalt!

Last but not least danke ich all den Patienten, die durch ihre umfangreiche Mitarbeit meine Arbeit erst ermöglichten!

Merci! 


\section{Lebenslauf}

$\begin{array}{ll}\text { Name: } & \text { Klosterhalfen } \\ \text { Vorname: } & \text { Stefanie Franziska } \\ \text { Anschrift: } & \begin{array}{l}\text { Eichenstr. 14 } \\ \text { 66450 Bexbach }\end{array} \\ \text { Telefon: } & \text { 0163-3332510, 06826-5756 } \\ \text { Geburtsdatum: } & 25.10 .1985 \\ \text { Geburtsort: } & \text { Neunkirchen (Saar) } \\ \text { Staatsangehörigkeit: } & \text { deutsch }\end{array}$

Schule und Studium:

Grundschule: $\quad 1992-1996$ Grundschule Niederbexbach

Gymnasium: $\quad 1996$ - 2005: Saarpfalz-Gymnasium Homburg (Saar) Abitur 2005

Studium: $\quad$ 2005-2011: Studium der Humanmedizin an der Medizinischen Fakultät der Universität des Saarlandes

1. Staatsexamen: Herbst 2007 (nach neuer Approbationsordnung)

2. Staatsexamen: Herbst 2011

Famulaturen: $\quad$ 08-09/2008 Praxis Fr. Dr. Maurer, Frauenärztin, Bexbach 03-04/2009 MediClin Bliestal Kliniken, Fachklinik für Psychosomatische Medizin und Psychotherapie, Blieskastel 09-10/2009 Anästhesiologie, Universitätsklinikum Homburg 03/2010 Frauenklinik, Universitätsklinikum Homburg

Praktisches Jahr:

1.Tertial: $\quad$ 08/2010-12/2010: Knappschaftskrankenhaus Sulzbach, Innere Medizin 2.Tertial: $\quad$ 12/2010-04/2011: Knappschaftskrankenhaus Sulzbach, Chirurgie 3.Tertial: $\quad$ 04/2011-07/2011: Universitätsklinikum Homburg, Wahlfach Gynäkologie und Geburtshilfe 


\section{Anhang \\ MediClinBliestal Kliniken}

MediClinBliestal Kliniken - Am Spitzenberg - 66440 Blieskastel
MEDICLIN

Fachklinik für

Psychosomatische Medizin

Chefarzt Prof. Dr. med. V. Köllner

Facharzt für Psychosomatische

Medizin und Psychotherapie

Am Spitzenberg

66440 Blieskastel

Telefon (06842)54-2257

Telefax (06842) 54-2340

Email:

koellner@bliestal.mediclin.de

www.mediclin.de

\title{
Forschungsvorhaben „Verbesserung des Transfers von Entspannungsverfahren in den Alltag der Patienten“"
}

\author{
Projektverantwortlich: Prof. Dr. med. Volker Köllner \\ Chefarzt der Fachklinik für \\ Psychosomatische Medizin \\ Bliestal Kliniken \\ 66440 Blieskastel
}

\section{Patienteninformation}

Sehr geehrte Patientin, sehr geehrter Patient,

Sie befinden sich zur stationären Rehabilitation in der Fachklinik für Psychosomatische Medizin.

Sie erhalten hier alle notwendigen und geeigneten Untersuchungen und Behandlungen, die zur wesentlichen Besserung oder Wiederherstellung Ihrer Gesundheit und Leistungsfähigkeit beitragen. Wir sind ständig bemüht, unser Behandlungsangebot den neuesten Erkenntnissen anzupassen und auch einen eigenen Beitrag hierzu zu leisten. Deshalb führen wir an unserer Klinik das Forschungsprojekt "Verbesserung des Transfers von Entspannungsverfahren in den Alltag der Patienten" durch. Wir möchten Sie hiermit bitten, uns und damit das Forschungsvorhaben zu unterstützen. Diese Teilnahme ist selbstverständlich freiwillig. Bevor Sie jedoch Ihr Einverständnis erteilen, erläutern wir Ihnen zunächst den geplanten Ablauf: 


\section{Beschreibung des Forschungsvorhabens:}

Entspannungsverfahren sind eine wirksame Möglichkeit, Körperzustände positiv zu beeinflussen und somit aktiv die eigene Gesundheit zu fördern. Sie stellen eine hochwirksame Behandlungsform von Erkrankungen dar, die mit erhöhter Anspannung in der Muskulatur oder dem vegetativen Nervensystem einhergehen. Hierzu gehören $u$. a. der Spannungskopfschmerz, andere chronische Schmerzzustände, Bluthochdruck und Schlafstörungen. Deshalb ist die Vermittlung eines Entspannungsverfahrens (in unserer Klinik Progressive Muskelentspannung nach Jacobson) in fast allen Rehabilitationskliniken ein fester Bestandteil des Behandlungsprogramms. Leider gelingt es aber nur einem Teil der Patienten, das in der Rehaklinik Gelernte in den häuslichen und beruflichen Alltag einzubauen. Somit bleibt diese quasi nebenwirkungsfreie Behandlungsmöglichkeit bei vielen Patienten ungenutzt. Wir haben in unserer Klinik deshalb ein neues Programm zur Vermittlung von Entspannungsverfahren entwickelt, das Patienten in besonderer Weise beim Transfer von der Entspannungsgruppe zuerst in den Alltag in der Klinik (Üben alleine während des Rehaaufenthalts) und später in den häuslichen Alltag unterstützen soll. Ziel dieses Forschungsvorhabens ist es, herauszufinden, ob dieses neue Programm gegenüber der bisherigen Durchführung des Entspannungstrainings wirklich eine Verbesserung darstellt und ob diese Verbesserung ausreichend ist, um möglichst vielen Patienten dabei zu helfen, Entspannungsverfahren auch nach der Entlassung aus der Klinik sicher und effektiv anzuwenden.

Zu diesem Zweck bitten wir Sie, vor Beginn des Entspannungstrainings einige Fragebögen zu Ihren Vorerfahrungen mit Entspannungsübungen, Ihrer Entspannungsfähigkeit sowie Ihren Beschwerden und Ihrem Gesundheitszustand auszufüllen.

Im weiteren Verlauf bitten wir Sie dann, nach jeder Entspannungsgruppe einen kurzen Beurteilungsbogen über die jeweilige Stunde auszufüllen. Nach Ablauf des gesamten Programms erfassen wir in einem ausführlicheren Fragebogen Ihre Einschätzung des Entspannungskurses, Ihre Einschätzung der nun gewonnenen Fähigkeit, sich zu entspannen sowie die bereits eingangs erfragten Beschwerden und die Angaben zum Gesundheitszustand.

Drei Monate nach der Entlassung werden wir Sie anschreiben und Ihnen einen weiteren Fragebogen zusenden. Hier geht es darum, wie gut es Ihnen gelungen ist, das Entspannungsverfahren in Ihren Alltag einzubauen und welchen Einfluss dies auf Ihre Beschwerden und Ihren Gesundheitszustand hat.

Durch unsere Klinik wurde vorab geprüft, ob Sie für die Teilnahme an dem Forschungsprojekt überhaupt in Frage kommen. Dies ist der Fall. Daraufhin wurden Sie von Ihrem behandelnden Klinikarzt oder vom Chefarzt der Klinik angesprochen. Sollten Sie sich für eine Teilnahme entscheiden, werden Ihre Daten in der oben beschriebenen Weise erhoben. Um die doppelte Abfrage von Daten zu vermeiden, bitten wir Sie um die Erlaubnis, auch die Daten, die im Rahmen Ihrer Behandlung in unserer Klinik erhoben wurden, für das Forschungsprojekt verwenden zu dürfen. Diese Daten werden von unserm Klinikpersonal bzw. von Frau cand. med. Stefanie Klosterhalfen aus der Krankenakte entnommen. Frau Klosterhalfen studiert Medizin an der Universitätsklinik Homburg/ Saar und führt dieses Forschungsprojekt im Rahmen ihrer Doktorarbeit durch. Die Doktorarbeit wird von Herrn Prof. Dr. med. V. Köllner persönlich betreut, so dass Ihre Daten unsere Klinik nicht verlassen. 


\section{Hinweise zum Datenschutz}

Für unser Forschungsprojekt spielt es keine Rolle, welchem Patienten (Name, Anschrift, Telefonnummer) die einzelnen Daten zugeordnet werden. Wie es die Datenschutzgesetze fordern, werden die für die Auswertung vorgesehenen Daten deshalb ohne Personenbezug streng getrennt von den personenbezogenen Daten aufbewahrt. Sobald Sie Ihre Teilnahme an dem Forschungsprojekt zusagen und die Einverständniserklärung unterschrieben haben, trägt ein mit der Durchführung des Forschungsvorhabens vertrauter Mitarbeiter unserer Klinik (Frau Klosterhalfen) Ihren Namen und Ihre Anschrift in eine fortlaufend nummerierte Liste ein. Diese Nummer (Forschungsnummer) vor dem Namen ist das Kennzeichen, unter dem ab jetzt alle Daten über Sie zusammengetragen werden. In den gesammelten Daten befinden sich somit keine Angaben, die direkt einen Bezug zu Ihrer Person herstellen lassen, z.B. Ihr Name.

Diese Zuordnungsliste verbleibt in unserer Klinik und ist nur Frau Klosterhalfen zugänglich. Sie wird weder den Klinikmitarbeitern noch anderen Stellen (z.B. der BfA) bekannt gegeben. Sie dient ausschließlich dem Ziel, den Kontakt mit Ihnen herzustellen und die über Sie gewonnenen Daten unter der Forschungsnummer zu sammeln. Am Ende der Datenerhebung, also nach etwa 1 Jahr, werden die personenbezogenen Daten (Zuordnungsliste und Einverständniserklärungen) vernichtet. So lässt sich auch in unserer Klinik keine Verbindung von den Forschungsnummern zu den Teilnehmern mehr herstellen. Ihre Angaben bleiben somit für das Klinikpersonal vollständig anonym. Ziel unseres Forschungsvorhabens ist es ja nicht, Ihr Verhalten zu überprüfen, sondern wir wollen die Effektivität unseres neuen Entspannungstrainings testen. Eine persönliche Zuordnung der Daten ist hierfür nicht erforderlich.

\section{Hinweise zur Freiwilligkeit}

Wenn Sie an diesem Forschungsvorhaben teilnehmen, können Sie jederzeit, auch bei schon gegebener Einwilligung, ohne Angabe von Gründen ausscheiden. Sofern Sie eine Teilnahme nicht wünschen, brauchen Sie die Einverständniserklärung nicht abzugeben. Weder aus der Teilnahme noch aus einer Nichtteilnahme erwachsen Ihnen Nachteile. Wie auch Ihre Entscheidung ausfallen mag, es wird auf die Durchführung der Rehabilitation keinen Einfluss haben.

Wenn Sie nicht an der Nachbefragung teilnehmen möchten, so senden Sie uns den Nachbefragungsbogen bitte im beiliegenden Freiumschlag unausgefüllt zurück. Wenn Sie es ausdrücklich wünschen, werden wir auch Ihre für die Forschung gesammelten Daten löschen und schriftliche Aufzeichnungen vernichten, soweit diese noch existieren.

Sie können sich darauf verlassen, dass Ihre Daten nur verwendet werden, wenn Sie die Einverständniserklärung unterschrieben haben.

Wenn Sie das Vorausgegangene gelesen haben, Ihnen der Inhalt klar ist und Sie an der Studie teilnehmen möchten, bitten wir Sie, die Einverständniserklärung zu unterschreiben und im Grundlagenseminar zur Entspannung zusammen mit den 
ersten beiden ausgefültten Fragebögen beim Seminarleiter abzugeben. Der Seminarleiter des Grundlagenseminars Entspannung wird Ihnen die Einverständniserklärung zusammen mit den ersten beiden Fragebögen im Grundlagenseminar aushändigen. Dieses Informationsschreiben können Sie in jedem Fall behalten. Sollten Sie nicht teilnehmen wollen, müssen Sie nichts unternehmen. Ihre Daten werden nur verwendet, wenn Sie eingewilligt haben. Dieses Forschungsvorhaben erfolgt im eigenen Interesse der Klinik und wurde nicht von der BfA veranlasst.

Für Rückfragen stehen Ihnen auch Ihre Bezugstherapeuten sowie die Leiter der Entspannungskurse gerne zur Verfügung. Selbstverständlich können Sie sich auch direkt an mich wenden.

Vielen Dank für Ihre Unterstützung!

Mit freundlichen Grüßen

Prof. Dr. med. Volker Köllner, Chefarzt der Fachklinik für

Psychosomatische Medizin 


\section{MEDICLIN}

Fachklinik für

Psychosomatische Medizin

Chefarzt Prof. Dr. med. V. Köllner

Facharzt für Psychosomatische

Medizin und Psychotherapie

Am Spitzenberg

66440 Blieskastel

Telefon (06842)54-2257

Telefax (06842) 54-2340

Email:

koellner@bliestal.mediclin.de

www.mediclin.de

\section{Einverständniserklärung}

Name:

Vorname:

Geburtsdatum:

Ich bin über den Inhalt und Zweck des Forschungsvorhabens „Verbesserung des Transfers von Entspannungsverfahren in den Altag der Patienten" informiert worden. Zu diesem Zwecke wurde mir ein Informationsschreiben ausgehändigt.

Mir wurde versichert, dass keine personenbezogenen Angaben (Name, Geburtsdatum, Adresse) oder sonstige Angaben, welche Rückschlüsse auf meine Person zulassen, an Dritte weitergegeben werden. Ich möchte das Forschungsvorhaben durch meine Beteiligung unterstützen.

Insbesondere bin ich damit einverstanden, dass Klinikmitarbeiter bzw. eine vom Chefarzt der Klinik betreute Doktorandin Diagnosen, Medikation und Behandlungsergebnisse aus der Krankenakte entnehmen und unter einer Forschungsnummer anonymisiert speichern. Ich entbinde die Klinikmitarbeiter insoweit von ihrer Schweigepflicht. Darüber hinaus stehe ich für telefonische Rückfragen nach meinem Klinikaufenthalt zur Verfügung.

Unter den in der Patienteninformation genannten Voraussetzungen erkläre ich mein Einverständnis für die Teilnahme an dem Forschungsvorhaben.

Ort:

Datum:

Unterschrift des Patienten: 
MediClinBliestal Kliniken, Blieskastel

Fachklinik für Psychosomatische Medizin

Dieses Kästchen wird vom Klinikpersonal ausgefüllt. Bitte nicht ausfüllen.

Eingangsdiagnose:

ICD-Diagnoseschlüssel:

Alter:

Geschlecht:

Forschungsnummer (Vpn):

Anreisedatum/Kalenderwoche:

Code Fragebogen:

\section{Krampen AT-EVA}

AT-ANAM Anamnese-Fragebogen für Teilnehmer (innen) an Entspannungstrainings und -therapien

1. Ihr Name:

2. Ihre Anschrift:

Telefon:

3. Ihr Geburtsdatum

4. Haben Sie Vorerfahrungen mit einer Entspannungstechnik oder -methode?

(bitte ankreuzen)

O a) ja, ich habe Autogenes Training schon einmal in einer Gruppe gelernt

- vor etwa Jahren

häufig selten nie

- zur Zeit praktiziere ich dies:

(1) (2) (3)

O b) ja, ich habe Autogenes Training in Einzelsitzungen (beim Arzt, Psychologen) gelernt

- vor etwa ___ Jahren

häufig selten nie

- zur Zeit praktiziere ich dies:

(1) (2) (3) 
O c) ja, ich habe mir Autogenes Training selbst (autodidaktisch) anhand von Büchern, Kassetten o.ä. beigebracht

- vor etwa Jahren

- zur Zeit praktiziere ich dies: (1) (2) (3)

häufig selten nie

O d) ja, ich habe die Progressive Muskelentspannungstechnik in einer Gruppe gelernt

- vor etwa Jahren

häufig selten nie

- zur Zeit praktiziere ich dies:

(1) (3)

O e) ja, ich habe mir die Progressive Muskelentspannungstechnik selbst anhand von Büchern, Kassetten o.ä. beigebracht

- vor etwa ___ Jahren

häufig selten nie

- zur Zeit praktiziere ich dies: (1) (2) (3)

O f) ja, ich habe Yoga oder eine andere Meditationstechnik gelernt

- vor etwa Jahren

häufig selten nie

- zur Zeit praktiziere ich dies: (1) (2) (3)

O g) ja, ich habe eine andere Entspannungstechnik gelernt

- welche Methode:?

- vor etwa Jahren

häufig selten nie

- zur Zeit praktiziere ich dies: (1) (2) (3)

O h) nein, ich habe bislang noch keine Entspannungsmethode gelernt 
5. Waren Sie schon einmal wegen einer Herz-Kreislauf-Erkrankung in ärztlicher Behandlung?

O nein

O ja: - wegen welcher Erkrankung?

- wann war das?

6. Waren Sie schon einmal in psychotherapeutischer, psychiatrischer oder neurologischer Behandlung?

O nein

O ja: - wegen welcher Erkrankung?

- wann war das?

7. Leiden Sie an einer chronischen Erkrankung?

O nein

O ja: - an welcher Erkrankung?

8. Nehmen Sie zur Zeit regelmäßig Medikamente? (bitte alle zutreffenden Medikamente ankreuzen)

O ja, Herz-Kreislauf-Medikamente

O ja, Schmerzmittel

O ja, Schlafmittel

O ja, Beruhigungsmittel

O ja, andere Medikamente - welche?

O nein, ich nehme keine Medikamente regelmäßig 
9. Warum nehmen Sie an einem Einführungskurs zu einer Entspannungsmethode teil? (Bitte alle zutreffenden Gründe ankreuzen!)

O a) weil es mir vom Arzt empfohlen wurde

O b) weil es mir vom Psychologen empfohlen wurde

O c) weil es mir von Freunden / Bekannten empfohlen wurde

O d) weil ich davon gehört habe und neugierig bin

O e) weil ich eine schwere akute Belastung verarbeiten muss

O f) weil ich mich besser entspannen und erholen können möchte

O g) weil ich ruhiger und gelassener werden möchte

O h) weil ich mich im Alltag weniger aufregen möchte

O i) weil ich mein Konzentrations- / Leistungsvermögen verbessern möchte

O j) weil ich meine Schmerzen in den Griff bekommen möchte

O k) weil ich den Wechsel zwischen körperlicher Aktivität und Ruhe lernen möchte

O I) weil ich meinen Körper besser kennenlernen möchte

O m) weil ich meine Gedanken positiv auf Entspannung und Ruhe ausrichten möchte

O n) weil ich mich auch in Anwesenheit anderer Personen besser entspannen und erholen können möchte

o) weil ich selbstsicherer werden möchte

O p) weil ich mich selbst besser kennenlernen möchte

O q) sonstige (weitere) Gründe: 
MediClinBliestal Kliniken, Blieskastel Fachklinik für Psychosomatische Medizin

Dieses Kästchen wird vom Klinikpersonal ausgefüllt. Bitte nicht ausfüllen.

Forschungsnummer (Vpn):

Meßzeitpunkt:

Code Fragebogen:

\section{Krampen AT-EVA AT-Sym Symptomfragebogen}

Ihr Name / Vorname:

Datum:

Bitte beantworten Sie diesen Beschwerdebogen sorgfältig und genau. Kreuzen Sie bitte jeweils an, in welchem Ausmaß Sie in den letzen 14 Tagen die jeweiligen Beschwerden, Schwierigkeiten, Gefühle oder Gedanken über sich selbst hatten. Verwenden Sie dabei die folgenden Antwortmöglichkeiten:

$$
\begin{aligned}
& 0=\text { trat bei Ihnen überhaupt nicht auf } \\
& 1=\text { trat bei Ihnen kaum (manchmal) auf } \\
& 2=\text { trat bei Ihnen häufiger / mäßig ausgeprägt auf } \\
& 3=\text { trat bei Ihnen sehr häufig / stark ausgeprägt auf }
\end{aligned}
$$

\begin{tabular}{|c|c|c|c|c|}
\hline & nie & & & stark \\
\hline 1. Gefühl, nicht abschalten zu können & (0) & (1) & (2) & (3) \\
\hline 2. Erschöpfung, Müdigkeit & (0) & (1) & (2) & (3) \\
\hline 3. Körperliche Verspannungen, Verkrampfungen & (0) & (1) & (2) & (3) \\
\hline 4. Innere Unruhe (Hektik, Gefühl des Gehetztseins) & (0) & (1) & (2) & (3) \\
\hline 5. Energielosigkeit (Schwächegefühl, Mattigkeit) & (0) & (1) & (2) & (3) \\
\hline 6. Ein- oder Durchschlafstörungen & (0) & (1) & (2) & (3) \\
\hline 7. Sich abgespannt und „gerädert" fühlen & (0) & (1) & (2) & (3) \\
\hline 8. Gefühl des Unwohlseins, Missbefinden & (0) & (1) & (2) & (3) \\
\hline 9. Reizbarkeit, leichte Erregbarkeit & (0) & (1) & (2) & (3) \\
\hline 10. Innere Anspannung, Nervosität & (0) & (1) & (2) & (3) \\
\hline 11. Gefühl der Unausgeglichenheit & (0) & (1) & (2) & (3) \\
\hline 12. Angstgefühle & (0) & (1) & (2) & (3) \\
\hline 13. Gefühl der Schwermütigkeit & (0) & (1) & (2) & (3) \\
\hline
\end{tabular}




$\begin{array}{lllll}\text { 14. Neigung zum Weinen } & \text { nie } & & \text { stark } \\ \text { 15. Trübe Gedanken } & \text { (?) } & \text { (1) (3) } \\ \text { 16. Leicht ärgerlich und verletzt sein } & \text { (0) (1) (2) (3) }\end{array}$

\begin{tabular}{|c|c|c|c|}
\hline 17. Schwindelgefühl, Kreislaufstörungen & (0) & (1) & (2) \\
\hline 18. Verdauungsstörungen & (0) & (1) & (2) \\
\hline 19. Appetitlosigkeit & (0) & (1) & (2) \\
\hline 20. Herzklopfen, -stolpern, -jagen & (0) & (1) & (2) \\
\hline 21. Atemnot, Luftmangel (bei Aufregung) & (0) & (1) & (2) \\
\hline 22. Kälteempfindlichkeit, Frieren, kalte Füße & (0) & (1) & (2) \\
\hline 23. Aufsteigende Hitze, Hitzewallungen, Schwitzen & (0) & (1) & (2) \\
\hline 24. Leichtes Erröten oder Zittern (bei Aufregung) & (0) & (1) & (2) \\
\hline
\end{tabular}

25. Angst vor Prüfungen / Leistungssituationen

() (1) (2) (3)

26. Konzentrationsschwierigkeiten, -schwächen

() (1) (2) (3)

27. Vergesslichkeit, Erinnerungsschwierigkeiten

() (1) (2) (3)

28. Mangelnde Ausdauer, Ablenkbarkeit

() (1) (2) (3)

29. Sprachschwierigkeiten (bei Aufregung)

(2) (1) (2) (3)

30. Leistungsschwäche, Lernschwierigkeiten

() (1) (2) (3)

31. Umgangsschwierigkeiten, Kontaktprobleme

() (1) (2) (3)

32. Gefühl, nicht zu leisten, was man könnte

(0) (1) (2) (3)

33. Kopfschmerzen, Druck im Kopf

(2) (1) (2) (3)

34. Bauchschmerzen, Magendruck, Unterleibsschmerzen

() (1) (2) (3)

35. Gelenk- oder Gliederschmerzen

(2) (1) (2) (3)

36. Nacken-, Kreuz- oder Rückenschmerzen

(7) (1) (2) (3)

37. Herzstiche, Herzschmerzen

() (1) (2) (3)

38. Ohrenschmerzen, Ohrgeräusche

() (1) (2) (3)

39. Augenflimmern, Schmerzen in den Augen

() (1) (2) (3)

40. Allgemeine, diffuse Schmerzen

(2) (1) (2) (3)

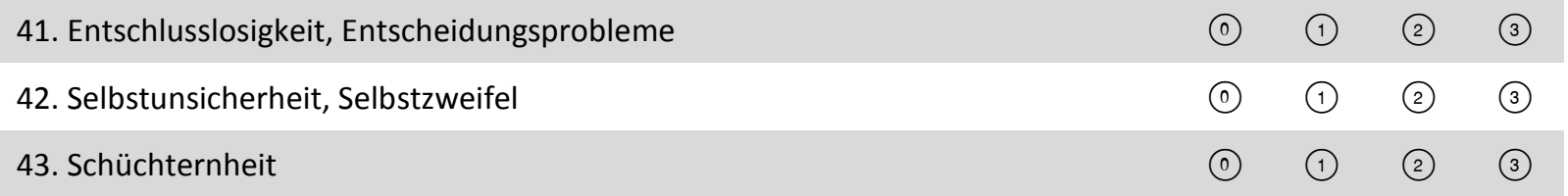




\begin{tabular}{|c|c|c|c|c|}
\hline & nie & & & stark \\
\hline 44. Mangelndes Selbstvertrauen & (0) & (1) & (2) & (3) \\
\hline 45. Angstzustände ohne Anlass & (0) & (1) & (2) & (3) \\
\hline 46. Gefühl, unausgefüllt zu sein & (0) & (1) & (2) & (3) \\
\hline 47. Hoffnungslos in die Zukunft blicken & (0) & (1) & (2) & (3) \\
\hline 48. Gefühl des gestörten inneren Gleichgewichts & (0) & (1) & (2) & (3) \\
\hline
\end{tabular}

Im Folgenden ist Raum gelassen für weitere Eintragungen, die sich auf Beschwerden und Probleme beziehen, die Ihnen wichtig erscheinen und bislang in diesem Fragebogen nicht angesprochen wurden:

nie

(2) (1) (2) (3)

49.

50.

51.

52.

53.

54.
(0) 1

(2) (1) (2) (3)

() (1) (2) (3)

(2) (1) (2) (3)

() (1) (2) (3)

Vielen Dank für Ihre Teilnahme! 
MediClinBliestal Kliniken, Blieskastel Fachklinik für Psychosomatische Medizin

Dieses Kästchen wird vom Klinikpersonal ausgefüllt. Bitte nicht ausfüllen.

Forschungsnummer (Vpn):

Meßzeitpunkt:

Code Fragebogen:

\section{Krampen AT-EVA}

\section{AT-STD Stundenbogen für die Progressive Muskelentspannung}

Insgesamt te Sitzung des Kurses Progressive Muskelentspannung

Ihr Name / Vorname: Datum:

Bitte beantworten Sie die folgenden Fragen zur heutigen Sitzung anhand der folgenden Antwortskala (bitte jeweils ankreuzen):

$$
\begin{aligned}
& 7=\text { ja, stimmt ganz genau } \\
& 6=\text { ja, stimmt } \\
& 5=\text { eher ja } \\
& 4=\text { weder ja, noch nein } \\
& 3=\text { eher nein } \\
& 2=\text { nein, stimmt nicht } \\
& 1=\text { nein, stimmt gar nicht }
\end{aligned}
$$

\begin{tabular}{|c|c|c|c|c|c|c|c|}
\hline & $\begin{array}{c}\text { Nein, } \\
\text { stimmt } \\
\text { gar } \\
\text { nicht }\end{array}$ & & & & & & $\begin{array}{c}\text { Ja, } \\
\text { stimm } \\
\text { ganz } \\
\text { genau }\end{array}$ \\
\hline 1. Während der Übung fühlte ich mich entspannt. & (1) & (2) & (3) & (4) & (5) & (6) & (7) \\
\hline 2. Die Übung dauerte mir zu lange. & (1) & (2) & (3) & (4) & (5) & (6) & (7) \\
\hline $\begin{array}{l}\text { 3. Ich fühlte mich durch die anderen Teilnehmer/innen } \\
\text { gestört. }\end{array}$ & (1) & (2) & (3) & (4) & (5) & (6) & (7) \\
\hline 4. Ich habe mich in der Gruppe wohl gefühlt. & (1) & (2) & (3) & (4) & (5) & (6) & (7) \\
\hline 5. Ich hatte Probleme mit der Körperhaltung. & (1) & (2) & (3) & (4) & (5) & (6) & (7) \\
\hline
\end{tabular}

6. Meine Arme waren angenehm entspannt.

(1)

(2) (3)

(3) (4)

5

(6)

(7)

7. Mein Schulter- Nacken- Bereich war angenehm entspannt.

(1) (2) (3) (4) (5) (6) 7)

8. Mein Gesicht war angenehm entspannt.

(1) (2)

(2) (3)

(4) (5)

(6)

9. Mein Rücken war angenehm entspannt.

(1) (2)

10. Mein Bauch-Bereich war angenehm entspannt.

(1) (2)

11. Meine Bein- und Gesäßmuskeln waren angenehm entspannt.

(2)

(3) (4)

(4) (5)


MediClinBliestal Kliniken, Blieskastel

Fachklinik für Psychosomatische Medizin

Dieses Kästchen wird vom Klinikpersonal ausgefüllt. Bitte nicht ausfüllen.

Forschungsnummer (Vpn):

Meßzeitpunkt:

Code Fragebogen:

\section{Krampen AT-EVA}

\section{AT-VFE Veränderungsfragebogen zum Entspannungserleben und Wohlbefinden}

Ihr Name / Vorname:

Datum:

Bitte stellen Sie sich die Zeit vor Beginn des Kurses zum Entspannungstraining vor. Das war vor etwa Wochen. Überlegen Sie, was Sie zu diesem Zeitpunkt machten und wie Sie sich fühlten.

Bitte prüfen Sie bei den nachfolgenden Aussagen immer, ob sich bei Ihnen seitdem - also im Verlauf der Teilnahme am Kurs - eine Veränderung in die eine oder andere Richtung vollzogen hat. Schätzen Sie diese Änderung jeweils anhand der folgenden Skala ein:

$$
\begin{aligned}
& 7=\text { starke } \text { positive Änderung } \\
& 6=\text { mittlere positive Änderung } \\
& 5=\text { schwache positive Änderung } \\
& 4=\text { keine Änderung } \\
& 3=\text { schwache negative Änderung } \\
& 2=\text { mittlere negative Änderung } \\
& 1=\text { starke negative Änderung }
\end{aligned}
$$

\begin{tabular}{|c|c|c|c|c|c|c|c|}
\hline & $\begin{array}{c}\text { Starke } \\
\text { neagative } \\
\text { Veránde- } \\
\text { rung }\end{array}$ & & & & & & $\begin{array}{c}\text { Starke } \\
\text { positive } \\
\text { Verände } \\
\text { rung }\end{array}$ \\
\hline $\begin{array}{l}\text { 1. Ich kann mich besser körperlich } \\
\text { entspannen. }\end{array}$ & (1) & (2) & (3) & (4) & (5) & (6) & (7) \\
\hline $\begin{array}{l}\text { 2. Ich kann besser abschalten und mich } \\
\text { erholen. }\end{array}$ & (1) & (2) & (3) & (4) & (5) & (6) & (7) \\
\hline 3. Ich fühle mich insgesamt gesunder. & (1) & (2) & (3) & (4) & (5) & (6) & (7) \\
\hline $\begin{array}{l}\text { 4. Ich bin weniger besorgt, wenn ich an die } \\
\text { Zukunft denke. }\end{array}$ & (1) & (2) & (3) & (4) & (5) & (6) & (7) \\
\hline 5. Ich fühle mich ruhiger und ausgeglichener. & (1) & (2) & (3) & (4) & (5) & (6) & (7) \\
\hline 6. Ich schlafe besser. & (1) & (2) & (3) & (4) & (5) & (6) & (7) \\
\hline 7. Ich nehme weniger Medikamente. & (1) & (2) & (3) & (4) & (5) & (6) & (7) \\
\hline $\begin{array}{l}\text { 8. Ich habe mehr Ausdauer und gebe nicht so } \\
\text { schnell auf. }\end{array}$ & (1) & (2) & (3) & (4) & (5) & (6) & (7) \\
\hline 9. Ich kann mich besser konzentrieren. & (1) & (2) & (3) & (4) & (5) & (6) & (7) \\
\hline $\begin{array}{l}\text { 10. Unerwartete Ereignisse nehme ich } \\
\text { gelassener hin. }\end{array}$ & (1) & (2) & (3) & (4) & (5) & (6) & (7) \\
\hline 11. Ich fühle mich wohler. & (1) & (2) & (3) & (4) & (5) & (6) & (7) \\
\hline $\begin{array}{l}\text { 12. Ich komme mit Stress und Belastungen } \\
\text { besser zurecht. }\end{array}$ & (1) & (2) & (3) & (4) & (5) & (6) & (7) \\
\hline
\end{tabular}


Abschließend noch einige Fragen zu den von Ihnen bisher durchgeführten Übungen:

13. Wie oft haben Sie in der Klinik außerhalb der Therapiestunden die Progressive Muskelentspannung selbständig geübt?

O täglich (oder öfter)

O etwa 2-3x pro Woche

O etwa 1x pro Woche

O etwa 2-3x während des gesamten Aufenthalts

O gar nicht

14. Haben Sie Hilfsmaterialien (Anleitungen, CD's, Broschüren) zu den Übungen erhalten?

O Ja

O Nein

15. Wenn ja, waren diese hilfreich?

O sehr

O etwas

O gar nicht

Wenn nein, hätten Sie sich Materialien gewünscht?

O Ja

O Nein

16. Wie empfanden Sie die Gruppengröße im Entspannungstraining?

O deutlich zu groß

O etwas zu groß

0 angemessen

O etwas zu klein

O deutlich zu klein

17. Welche Anregungen und Verbesserungsvorschläge haben Sie für unser Entspannungstraining? 
MediClinBliestal Kliniken, Blieskastel

Fachklinik für Psychosomatische Medizin

Dieses Kästchen wird vom Klinikpersonal ausgefüllt. Bitte nicht ausfüllen.

Forschungsnummer (Vpn):

Code Fragebogen:

\section{Krampen AT-EVA}

\section{AT-KATAM Nachbefragungsbogen für Teilnehmer(innen) an Entspannungstrainings und -therapien}

Ihr Name / Vorname: Datum:

Alter:

Geschlecht: O weiblich,

O männlich

Bitte beantworten Sie die folgenden Fragen sorgfältig und genau. Kreuzen Sie jeweils die Antwortalternative an, die auf Sie persönlich am besten zutrifft.

Wegen welcher Diagnose wurden Sie in den Bliestal Kliniken behandelt?
1. Wie häufig wenden sie das Entspannungstraining zur Zeit etwa an?
O mehrmals am Tag
O etwa 1x pro Tag
O mehrmals pro Woche
O 1 bis $2 x$ pro Woche
O mehrmals im Monat
O etwa 1x im Monat
O seltener
O seit etwa Monaten nicht mehr
O seit dem Einführungskurs nicht mehr 
2. Welche Meinung haben Sie heute über das Entspannungstraining (ET)?

Nein

$\mathrm{Ja}$

\begin{tabular}{|c|c|c|c|c|c|c|}
\hline a) Das Entspannungstraining hat mir persönlich genutzt & (1) & (2) & (3) & (4) & (5) & (6) \\
\hline b) Das Entspannungstraining verspricht mehr als es hält & (1) & (2) & (3) & (4) & (5) & (6) \\
\hline $\begin{array}{l}\text { c) Durch das Entspannungstraining kann ich mich besser } \\
\text { entspannen }\end{array}$ & (1) & (2) & (3) & (4) & (5) & (6) \\
\hline $\begin{array}{l}\text { d) Das Entspannungstraining hat mir schon bei einem persönlichen } \\
\text { Problem geholfen }\end{array}$ & (1) & (2) & (3) & (4) & (5) & (6) \\
\hline $\begin{array}{l}\text { e) Mit dem Entspannungstraining kann ich persönlich nicht viel } \\
\text { anfangen }\end{array}$ & (1) & (2) & (3) & (4) & (5) & (6) \\
\hline $\begin{array}{l}\text { f) Ich würde das Entspannungstraining anderen Menschen } \\
\text { empfehlen }\end{array}$ & (1) & (2) & (3) & (4) & (5) & (6) \\
\hline
\end{tabular}

g) Ich habe das Entspannungstraining schon anderen empfohlen

O ja

0 nein

3. Schätzen Sie bitte auf der folgenden Skala Ihr aktuelles persönliches Befinden ein:

Ich fühle mich zur Zeit ...

sehr unwohl (1) (2) (3) (4) (5) (6) (7) sehrwohl

4. Falls Sie das Entspannungstraining noch anwenden: In welcher Körperhaltung führen Sie die Übungen meistens durch?

O sitzend auf einem Stuhl in der Droschkenkutscherhaltung

O zurückgelehnt sitzend in einem Sessel o.ä.

$\mathrm{O}$ in der Rückenlage liegend

5. Falls Sie das Entspannungstraining noch anwenden: Welche Wirkungen stellen sich dabei in der Regel bei Ihnen ein?

\begin{tabular}{|c|c|c|c|c|c|c|c|}
\hline & $\begin{array}{l}\text { Schlechte } \\
\text { Wirkung }\end{array}$ & & & & & & $\begin{array}{c}\text { Gute } \\
\text { Wirkung }\end{array}$ \\
\hline a) Meine Arme sind angenehm entspannt & (1) & (2) & (3) & (4) & (5) & (6) & (7) \\
\hline $\begin{array}{l}\text { b) Mein Schulter-Nackenbereich ist angenehm } \\
\text { entspannt }\end{array}$ & (1) & (2) & (3) & (4) & (5) & (6) & (7) \\
\hline c) Mein Gesicht ist angenehm entspannt & (1) & (2) & (3) & (4) & (5) & (6) & (7) \\
\hline d) Mein Rücken ist angenehm entspannt & (1) & (2) & (3) & (4) & (5) & (6) & (7) \\
\hline e) Mein Bauchbereich ist angenehm entspannt & (1) & (2) & (3) & (4) & (5) & (6) & (7) \\
\hline $\begin{array}{l}\text { f) Meine Bein- und Gesäßmuskeln sind angenehm } \\
\text { entspannt }\end{array}$ & (1) & (2) & (3) & (4) & (5) & (6) & (7) \\
\hline
\end{tabular}


Nun noch einige Fragen dazu, wie Sie das Entspannungstraining in unserer Klinik erlebt haben:

6. Welche Note von 1 (sehr gut) bis 6 (ungenügend) würden Sie dem Entspannungstraining insgesamt geben? sehrgut (1) (2) (3) (4) (5) (6) ungenügend

7. Wie würden Sie den Inhalt des Vortrags zur Entspannung (Grundlagenseminar) beurteilen?

O viel zu schwierig

O etwas zu schwierig

0 gerade richtig

O etwas zu einfach

O viel zu einfach

8. Welche Note von 1 (sehr gut) bis 6 (ungenügend) würden Sie dem Vortragsstil des Vortrags zur Entspannung (Grundlagenseminar) geben?

sehrgut (1) (2) (3) (4) (5) (6) ungenügend

9. Wurden Sie vom Kursleiter ermutigt, die Übungen selbst auszuprobieren?

O ja, regelmäßig

O ja, gelegentlich

0 nein

O ich kann mich nicht erinnern

10. Gab es im Kurs Gelegenheit, über die eigenen Erfahrungen beim selbständigen Üben zu sprechen?

O ja, regelmäßig

O ja, gelegentlich

0 nein

O ich kann mich nicht erinnern

11. Erhielten Sie vom Kursleiter Materialien, die Ihnen beim selbständigen Üben helfen sollten?

O ja, eine CD

O ja, schriftliches Informationsmaterial

O nein

Wenn ja, welche Note von 1 (sehr gut) bis 6 (ungenügend) würden Sie diesen Materialien geben?

sehrgut (1) (2) (3) (4) (5) (6) ungenügend 
Wenn nein, hätten Sie sich Materialien gewünscht?

O ja, eine $C D$

O ja, schriftliches Informationsmaterial

O nein

12. Wie beurteilen Sie die Gruppengröße beim Entspannungstraining in unserer Klinik?

O viel zu groß

O etwas zu groß

O genau angemessen

O etwas zu klein

O viel zu klein

13. Welche Note von 1 (sehr gut) bis 6 (ungenügend) würden Sie der Therapie in den Bliestal Kliniken insgesamt (also Gesamtheit der ärztlichen, psychologischen, physiotherapeutischen ... Behandlungsmaßnahmen) geben?

sehr gut (1) (2) (3) (4) (5) (6) ungenügend

14. Platz für Anmerkungen, die Ihnen wichtig sind und die Sie uns mitteilen möchten:

\section{Vielen Dank für Ihre Teilnahme!}




\section{MediClinBliestal Kliniken}

MediClinBliestal Kliniken - Am Spitzenberg -66440 Blieskastel
MEDICLIN

\author{
Fachklinik für \\ Psychosomatische Medizin
}

Chefarzt Prof. Dr. med. V. Köllner Facharzt für Psychosomatische Medizin und Psychotherapie

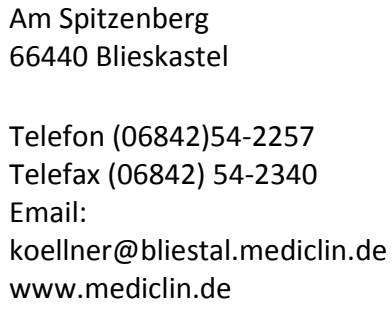

Sehr geehrte Patientin,

Sehr geehrter Patient,

vor etwa 3 Monaten waren Sie in unserer Fachklinik in stationärer Behandlung. Hierbei wurde den meisten von Ihnen auch ein Entspannungsverfahren vermittelt. Für den Erfolg einer stationären Rehabilitation ist es entscheidend, dass es gelingt, in den Altag zu Hause umzusetzen, was in der Rehabilitationsklinik gelernt wurde. Deshalb bemühen wir uns, unsere therapeutischen Konzepte gerade in dieser Hinsicht weiter zu verbessern.

Grundlage für solche Verbesserungen sind die Erfahrungen mit dem bisherigen Behandlungsprogramm. An dieser Stelle benötigen wir Ihre Mithilfe. Ich bitte Sie deshalb, den beiliegenden Fragebogen zum Thema Entspannungsverfahren sorgfältig auszufüllen und in dem ebenfalls beiliegenden Freiumschlag an uns zurückzusenden.

Wenn Sie nicht an der Befragung teilnehmen möchten, so können Sie uns dies dadurch mitteilen, dass Sie den unausgefüllten Fragebogen im Freiumschlag zurückschicken. Wenn Sie nicht an einem Entspannungskurs teilgenommen haben, schreiben Sie bitte „Nicht teilgenommen“ auf den Fragebogen und senden ihn ebenfalls leer zurück.

Ich möchte Sie darauf hinweisen, dass die Teilnahme an der Studie absolut freiwillig ist. Es geht auch nicht darum, Sie zu überprüfen.

Sie haben während Ihres Klinikaufenthalts bereits an diesem Forschungsprojekt teilgenommen und Fragebögen im Verlauf des Entspannungstrainings ausgefüllt. Bei dieser letzen Befragung wird wie bisher verfahren: Ein Ihnen bereits bekannter und vertrauter Mitarbeiter unserer Klinik (Frau Klosterhalfen) trug Ihren Namen und Ihre Anschrift in eine fortlaufend nummerierte Liste ein. Unter dieser Nummer (Forschungsnummer) wurden bisher alle Daten über Sie zusammengetragen und werden nun durch diese Angaben ergänzt. In den gesammelten Daten befinden sich keine Angaben, die direkt einen Bezug zu Ihrer Person herstellen lassen. Am Ende der Erhebung werden die personenbezogenen Daten (Zuordnungsliste und Einverständniserklärung) vernichtet. Ihre Angaben bleiben somit für das Klinikpersonal vollständig anonym. 
Ich bitte Sie aber sehr darum, uns zu unterstützen, weil wir mit Hilfe Ihrer Angaben unser Entspannungstraining für zukünftige Patienten (und vielleicht ja auch für Sie, wenn Sie noch einmal an einer stationären REHA-Maßnahme teilnehmen) verbessern können.

Das Ausfüllen des Fragebogens kann darüber hinaus ja auch eine gute Gelegenheit sein, noch einmal darüber nachzudenken, ob Sie auf dem richtigen Weg sind, die Dinge umzusetzen, die Sie sich bei der Entlassung aus unserer Klinik vorgenommen haben.

Wenn Sie zu unserer Studie oder zu Ihrem Aufenthalt in unserer Klinik Fragen haben, können Sie sich gerne an mich wenden.

Mit freundlichen Grüßen

Prof. Dr. med. V. Köllner

Chefarzt 


\section{Erklärung gemäß $§ 7$ Abs. 1 Nr. 4}

Ich erkläre hiermit an Eides statt, dass ich die vorliegende Arbeit ohne unzulässige Hilfe Dritter und ohne Benutzung anderer als der angegebenen Hilfsmittel angefertigt habe. Die aus anderen Quellen direkt oder indirekt übernommenen Daten und Konzepte sind unter Angabe der Quelle gekennzeichnet.

Bei der Auswahl und Auswertung folgenden Materials haben mir die nachstehend aufgeführten Personen in der jeweils beschriebenen Weise unentgeltlich/entgeltlich geholfen: 1. Prof. Dr. med. habil. V. Köllner

2. Dr. Nicolas Becker

Weitere Personen waren an der inhaltlich-materiellen Erstellung der vorliegenden Arbeit nicht beteiligt. Insbesondere habe ich nicht die entgeltliche Hilfe von Vermittlungs- bzw. Beratungsdiensten (Promotionsberater/innen oder anderer Personen) in Anspruch genommen. Außer den Angegebenen hat niemand von mir unmittelbar oder mittelbar geldwerte Leistungen für Arbeiten erhalten, die im Zusammenhang mit dem Inhalt der vorgelegten Dissertation stehen.

Die Arbeit wurde bisher weder im Inland noch im Ausland in gleicher oder ähnlicher Form in einem anderen Verfahren zur Erlangung des Doktorgrades einer anderen Prüfungsbehörde vorgelegt.

Ich versichere an Eides statt, dass ich nach bestem Wissen die Wahrheit gesagt und nichts verschwiegen habe.

Vor Aufnahme der vorstehenden Versicherung an Eides Statt wurde ich über die Bedeutung einer eidesstattlichen Versicherung und die strafrechtlichen Folgen einer unrichtigen oder unvollständigen eidesstattlichen Versicherung belehrt.

Ort, Datum

Unterschrift der/des Promovierenden

Unterschrift der die Versicherung an Eides statt aufnehmenden Beamtin bzw. des aufnehmenden Beamten 